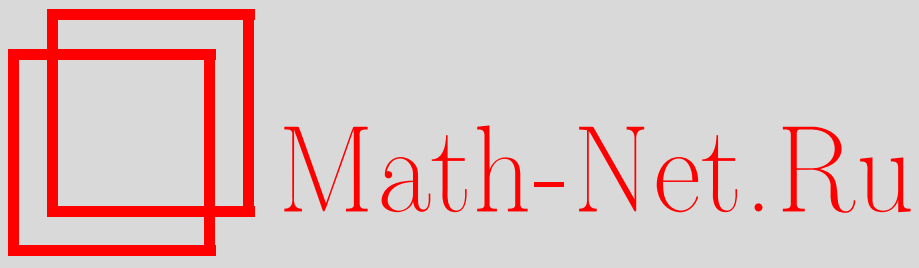

О. А. Ладыженская, Г. А. Серёгин, О гладкости решений систем, описывающих течения обобщенных ньютоновских жидкостей, и об оценке размерностей их аттракторов, Изв. РАН. Сер. матем., 1998, том 62, выпуск 1, 59-122

DOI: https://doi.org/10.4213/im183

Использование Общероссийского математического портала Math-Net.Ru подразумевает, что вы прочитали и согласны с пользовательским соглашением

http://www.mathnet.ru/rus/agreement

Параметры загрузки:

IP: 54.224 .135 .184

26 апреля 2023 г., 06:05:42 
УДК $517.9+534.231$

\author{
О. А. Ладыженская, Г. А. Серегин
}

\title{
О гладкости решений систем, описывающих течения обобщенных ньютоновских жидкостей, и об оценке размерностей их аттракторов
}

\begin{abstract}
Исследуются двумерные начально-краевые задачи динамики обобщенных ньютоновских жидкостей. Доказываются результаты по гладкости решений таких задач и оценкам хаусдорфовой и фрактальной размерностей их аттракторов.

Библиография: 17 наименований.
\end{abstract}

\section{§ 1. Постановка задачи и формулировка основных результатов}

Пусть $D$ - известная скалярная функция, заданная на множестве симметричных тензоров второго ранга. Для фиксированных положительного числа $T$, области $\Omega$ в $\mathbb{R}^{2}$ и векторного поля $g$ на $\left.Q_{T} \equiv \Omega \times\right] 0, T\left[\right.$ рассмотрим в $Q_{T}$ следующую систему дифференциальных уравнений в частных производных:

$$
\begin{gathered}
\partial_{t} v+(\nabla v) v-\operatorname{div} \sigma=g-\nabla p, \\
\operatorname{div} v=0, \\
\sigma=\frac{\partial D}{\partial \varepsilon}(\varepsilon(v)), \\
\varepsilon(v)-\text { симметричная часть градиента } \nabla v,
\end{gathered}
$$

в которой неизвестными являются векторное поле $v$ и скалярная функция $p$.

В случае, когда потенциал $D$ зависит только от второго инварианта своего аргумента, т.е. $D(\varepsilon)=D_{0}\left(|\varepsilon|^{2}\right)$, где $\varepsilon-$ симметричный тензор второго ранга и $|\varepsilon|=\sqrt{\operatorname{tr} \varepsilon^{2}}$, система $\left(1.1_{k}\right), k=1,2,3,4$, описьвает течение несжимаемой обобщенной ньютоновской жидкости с нелинейной вязкостью $\mu_{0}\left(|\varepsilon|^{2}\right)=2 D_{0}^{\prime}\left(|\varepsilon|^{2}\right)$, поскольку

$$
\sigma=\frac{\partial D}{\partial \varepsilon}(\varepsilon(v))=\mu_{0}\left(|\varepsilon(v)|^{2}\right) \varepsilon(v) .
$$

При этом функции $v$ и $р$ имеют физический смысл вектора скорости и давления.

Работа выполнена при частичной поддержке РФФИ, грант № 96-01-00824, и INTAS, грант № 94-1376.

(C) О.А. ЛАДЫЖЕНСКАЯ, Г.А. СЕРеГИН, 1998 
Система $\left(1.1_{k}\right), k=1,2,3,4$, записана в инвариантной форме, не зависящей от выбора конкретной системы координат. Пусть $x_{k}, k=1,2,-$ произвольная фиксированная ортогональная декартова система координат в $\mathbb{R}^{2}$. Используя координатные представления векторов и тензоров относительно выбранной системы координат, мы будем отождествлять векторы и симметричные тензоры второго ранга с элементами пространств $\mathbb{R}^{2}$ и $\mathbb{M}$ соответственно. Здесь $\mathbb{M}$ - пространство вещественных симметричных $(2 \times 2)$-матриц. Таким образом, $v=\left(v_{i}\right), \tau=\left(\tau_{i j}\right)$ и т.д., где $v_{i}$ и $\tau_{i j}$ - координаты вектора $v$ и тензора $\tau$ в данной декартовой системе координат. Примем соглашение о суммировании по повторяющимся латинским индексам и положим

$$
\begin{gathered}
a \otimes b=\left(a_{i} b_{j}\right), \quad a \odot b=\frac{1}{2}(a \otimes b+b \otimes a), \\
a \cdot b=a_{i} b_{i}, \quad|a|=\sqrt{a \cdot a}, \quad \sigma a=\left(\sigma_{i j} a_{j}\right), \\
\sigma: \varepsilon=\operatorname{tr} \sigma \varepsilon=\sigma_{i j} \varepsilon_{i j}, \quad|\sigma|=\sqrt{\sigma: \sigma}, \quad \frac{\partial D}{\partial \varepsilon}(\varepsilon)=\left(\frac{\partial D}{\partial \varepsilon_{i j}}(\varepsilon)\right)
\end{gathered}
$$

для $a, b \in \mathbb{R}^{2}$ и $\sigma, \varepsilon \in \mathbb{M}$.

Обозначим через $f_{, k}$ частную производную от функции $f$ по переменной $x_{k}$, т.е. $f_{, k} \equiv \frac{\partial f}{\partial x_{k}}$. Тогда для дифференциальных операторов $\nabla$ и $\operatorname{div}$ мы будем иметь представления

$$
\begin{aligned}
\nabla p=\left(p,,_{i}\right), \quad \nabla v & =\left(v_{i, j}\right), \quad \operatorname{div} v=v_{i, i}, \\
\operatorname{div} \sigma & =\left(\sigma_{i j, j}\right)
\end{aligned}
$$

и, таким образом,

$$
(\nabla v) v=v_{k} v_{, k}=\left(v_{i, k} v_{k}\right), \quad \varepsilon(v)=\frac{1}{2}\left(v_{i, j}+v_{j, i}\right) .
$$

Система $\left(1.1_{k}\right), k=1,2,3,4$, должна быть дополнена начальными и краевыми условиями. В данной работе мы ограничимся случаем периодических краевых условий, предполагая, что $\Omega=\left\{x \in \mathbb{R}^{2}:\left|x_{k}\right|<1, k=1,2\right\}$ - квадрат. Итак,

$$
\left.v\right|_{t=0}=\varphi \quad \text { в } \Omega
$$

и

$$
\begin{gathered}
\left.v\right|_{x_{k}=-1}=\left.v\right|_{x_{k}=1},\left.\quad \nabla v\right|_{x_{k}=-1}=\left.\nabla v\right|_{x_{k}=1}, \\
\left.p\right|_{x_{k}=-1}=\left.p\right|_{x_{k}=1} .
\end{gathered}
$$

Начально-краевая задача $\left(1.1_{k}\right), k=1,2, \ldots, 6$, будет изучаться при следуюших предположениях относительно потенциала $D$ :

$$
\begin{gathered}
D-\text { функция класса } C^{2} \text { и } D(0)=0, \\
D(\varepsilon) \geqslant \nu|\varepsilon|^{2}, \quad \nu>0-\text { const, } \\
\left(\frac{\partial^{2} D}{\partial \varepsilon^{2}}(\varepsilon) \varkappa\right): \varkappa \equiv \frac{\partial^{2} D}{\partial \varepsilon_{i j} \partial \varepsilon_{k m}}(\varepsilon) \varkappa_{i j} \varkappa_{k m} \geqslant 2 \nu|\varkappa|^{2}, \\
\left|\frac{\partial D}{\partial \varepsilon}(\varepsilon)\right| \leqslant \nu_{1}(|\varepsilon|+1), \quad \nu_{1}-\text { const },
\end{gathered}
$$


для всех $\varepsilon, \varkappa \in \mathbb{M}$.

При $D(\varepsilon)=\nu|\varepsilon|^{2}$ уравнения $\left(1.1_{k}\right), k=1,2,3,4$, совпадают с уравнениями Навье-Стокса.

Для того чтобы аккуратно формулировать результаты по сушествованию и регулярности решений начально-краевой задачи $\left(1.1_{k}\right), k=1,2, \ldots, 6$, введем следующие функциональные пространства:

$L_{p}\left(\omega ; \mathbb{R}^{k}\right), W_{p}^{l}\left(\omega ; \mathbb{R}^{k}\right)$ - известные пространства Лебега и Соболева функций, заданных на $\omega \in \mathbb{R}^{m}$, со значениями в $\mathbb{R}^{k}$. Норму в пространстве $L_{p}\left(\omega ; \mathbb{R}^{k}\right)$ будем обозначать символом $\|\cdot\|_{p, \omega}$, причем если $p=2$, то $\|\cdot\|_{\omega} \equiv\|\cdot\|_{2, \omega}$, если $\omega=\Omega$, то $\|\cdot\|_{p} \equiv\|\cdot\|_{p, \Omega}$, и если $p=2$ и $\omega=\Omega$, то $\|\cdot\| \equiv\|\cdot\|_{2, \Omega}$. Скалярное произведение в $L_{2}\left(\omega ; \mathbb{R}^{k}\right)$ обозначется через $(\cdot, \cdot) \omega$, а при $\omega=\Omega$ через $(\cdot, \cdot)$;

$L_{p}(0, T ; B)$ - пространство измеримых функций на отрезке $[0, T]$ со значениями в банаховом пространстве $B$, имеюших конечную норму $\left(\int_{0}^{T}\|f(t)\|_{B}^{p} d t\right)^{1 / p}$, если $1 \leqslant p<+\infty$, и ess $\sup _{0 \leqslant t \leqslant T}\|f(t)\|_{B}$, если $p=+\infty$

$C([0, T] ; B)$ - пространство непрерьвных на отрезке $[0, T]$ функций со значениями в банаховом пространстве $B$;

$W_{2, \text { per }}^{1}\left(\Omega ; \mathbb{R}^{m}\right)=\left\{f \in W_{2}^{1}\left(\Omega ; \mathbb{R}^{m}\right):\left.f\right|_{x_{k}=-1}=\left.f\right|_{x_{k}=1}, k=1,2\right\} ;$

$\stackrel{\circ}{W}_{2, \text { per }}^{1}\left(\Omega ; \mathbb{R}^{m}\right)=\left\{f \in W_{2, \text { per }}^{1}\left(\Omega ; \mathbb{R}^{m}\right): \int_{\Omega} f d x=0\right\}$;

$\hat{J}^{\infty}$ - пространство ограничений на $\Omega$ бесконечно дифференцируемых соленоидальных функций $v: \mathbb{R}^{2} \rightarrow \mathbb{R}^{2}$, удовлетворяюших условию периодичности $v(x+$ $2 z)=v(x) \forall x \in \Omega$ и $z \in Z^{2}$ и условию нормировки $\int_{\Omega} v d x=0$;

$H$ - замыкание множества $\hat{J}^{\infty}$ в $L_{2}\left(\Omega ; \mathbb{R}^{2}\right)$, снабжаемое нормой $\|\cdot\|$ и скалярным произведением $(\cdot, \cdot)$ пространства $L_{2}\left(\Omega ; \mathbb{R}^{2}\right)$;

$H^{1}$ - замыкание множества $\hat{J}^{\infty}{ }_{\text {в }} W_{2}^{1}\left(\Omega ; \mathbb{R}^{2}\right)$, снабжаемое нормой $\|v\|_{(1)}=\|\nabla v\|$;

$H^{-1}$ - пространство, двойственное к $H^{1}$ относительно $H$, с нормой $\|v\|_{(-1)}=$ $\sup \left\{(v, u):\|u\|_{(1)}=1\right\}$

$H^{2}$ - замыкание множества $\hat{J}^{\infty}$ в $W_{2}^{2}\left(\Omega ; \mathbb{R}^{2}\right)$, снабжаемое нормой $\|v\|_{(2)}=\|\Delta v\|$.

Обозначим через $\lambda_{k}, k=1,2, \ldots$, собственные значения оператора Стокса при периодических краевых условиях, т.е.

$$
\left(\nabla \varphi^{k}, \nabla w\right)=\lambda_{k}\left(\varphi^{k}, w\right) \quad \forall w \in H^{1}
$$

где $\varphi^{k} \in H^{1}, k=1,2, \ldots,-$ соответствуюшие собственные функции, удовлетворяющие условиям $\left\|\varphi^{k}\right\|=1, k=1,2, \ldots$, и $\left(\varphi^{k}, \varphi^{l}\right)=0, k, j=1,2, \ldots, k \neq j$.

Глобальная однозначная разрешимость начально-краевой задачи $\left(1.1_{k}\right)$, $k=1,2, \ldots, 5$, с однородными условиями Дирихле $\left.v\right|_{\partial \Omega}=0$ в классе обобщенных решений с конечной энергетической нормой была доказана в работах [1]-[3] одного из авторов. В [4], [5] для нее доказано свойство мгновенного сглаживания, существование компактного минимального глобального $B$-аттрактора $\mathfrak{M}$ и ряд его важных свойств. Для краевых условий $\left(1.1_{6}\right)$ все делается аналогично, и даже проше. Суммируя результаты работ [1]-[5], сформулируем следующие теоремы. 
ТЕОРема 1.1. 1) Пусть выполнены условия $\left(1.2_{k}\right), \quad k=1,2,3,4$. Тогда для всех $T>0, \varphi \in H$ u $g \in L_{2}\left(0, T ; H^{-1}\right)$ начально-краевая задача $\left(1.1_{k}\right)$, $k=1,2, \ldots, 6$, имеет единственное решение $v$, обладающее следующими свойствами:

$$
v \in C([0, T] ; H) \cap L_{2}\left(0, T ; H^{1}\right), \quad \partial_{t} v \in L_{2}\left(0, T ; H^{-1}\right) .
$$

Оно удовлетворяет для п.в. $t \in[0, T]$ вариационному тождеству

$$
\left(\partial_{t} v(t), w\right)+(\nabla v(t) v(t), w)+\left(\frac{\partial D}{\partial \varepsilon}(\varepsilon(v(t))), \varepsilon(w)\right)=(g(t), w) \quad \forall w \in H^{1}
$$

и оиенке

$$
\sup _{0 \leqslant t \leqslant T}\|v(t)\|^{2}+\int_{0}^{T}\left(\|\nabla v(t)\|^{2}+\left\|\partial_{t} v(t)\right\|_{(-1)}^{2}\right) d t \leqslant \Phi_{1}\left(T,\|\varphi\|,\|g\|_{L_{2}\left(0, T ; H^{-1}\right)}\right) .
$$

Здесь и всюду в дальнейшем мажоранты $\Phi_{i}$ суть непрерывные и неубывающие функиии своих аргументов.

2) Если дополнительно предположить, что

$$
t \partial_{t} g \in L_{2}\left(0, T ; H^{-1}\right)
$$

$m o \sqrt{t} v \in L_{\infty}\left(0, T ; H^{1}\right), \sqrt{t} \partial_{t} v \in L_{2}\left(Q_{T} ; \mathbb{R}^{2}\right), \quad t \partial_{t} v \in L_{\infty}(0, T ; H) \cap L_{2}\left(0, T ; H^{1}\right)$ и верна оченка

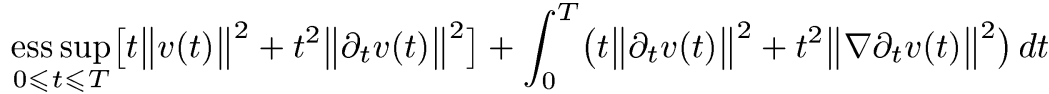

$$
\begin{aligned}
& \leqslant \Phi_{2}\left(T,\|\varphi\|,\|g\|_{L_{2}\left(0, T ; H^{-1}\right)},\left\|t \partial_{t} g\right\|_{L_{2}\left(0, T ; H^{-1}\right)}\right) .
\end{aligned}
$$

Как правило, за исключением специально оговоренных случаев, мы не будем следить, как наши мажоранты в оценках зависят от потенциала $D$.

ТеОрема 1.2. Пусть выполнены условия $\left(1.2_{k}\right), \quad k=1,2,3,4$, и пусть дополнительно

$$
g(t) \equiv f \in H^{-1} .
$$

Тогда задача $\left(1.1_{k}\right), \quad k=1,2, \ldots, 6$, однозначно разрешима для всех $T>0$ $u \varphi \in H$, а ее разрешающие операторы $V_{t}: H \rightarrow H\left(V_{t}(\varphi) \equiv v(t)\right), \quad t \geqslant 0$, образуют полугруппу класса $I$, имеющую $B$-поглощающие множества вида

$$
\{u \in H:\|u\|<R\}, \quad R>R_{0}=\frac{2\|f\|_{(-1)}}{\nu \sqrt{\lambda_{1}}} .
$$


Эта полугруппа имеет компактный минимальный глобальный $B$-аттрактор $\mathfrak{M}$, который является связным инвариантным множеством в $H$, ограниченным в $H^{1}$. Для любого $\varphi \in \mathfrak{M}$ решение $V_{t}(\varphi), t \in \mathbb{R}_{+}^{1}=\left\{q \in \mathbb{R}^{1}: q \geqslant 0\right\}$, может быть продолжено на $\mathbb{R}_{-}^{1}=\left\{q \in \mathbb{R}^{1}: q<0\right\}$ так, чтобы $V_{t}(\varphi)$ было решением системы $\left(1.1_{k}\right), \quad k=1,2,3,4$, для всех $t \in \mathbb{R}^{1}$. Более того, имеет место оценка

$$
M_{1}=\sup _{\varphi \in \mathfrak{M}} \sup _{t \in \mathbb{R}^{1}}\left\{\left\|\nabla V_{t}(\varphi)\right\|^{2}+\left\|\partial_{t} V_{t}(\varphi)\right\|^{2}+\int_{t}^{t+1}\left\|\nabla \partial_{\theta} V_{\theta}(\varphi)\right\|^{2} d \theta\right\}<+\infty .
$$

Число определяющих мод на $\mathfrak{M}$ конечно.

При формулировке теоремы 1.2 мы использовали терминологию из [6]. По поводу более ранних результатов, связанных с двумерными уравнениями Навье-Стокса, мы отсылаем читателя к работе [7].

В [4], [5] мы не сумели доказать конечномерность аттрактора $\mathfrak{M}$, поскольку не обладали необходимой для этого информацией о дифференциальных свойствах решения задачи $\left(1.1_{k}\right), k=1,2, \ldots, 6$. Некоторый прогресс в этом направлении был достигнут в [8], однако все еще недостаточньй для получения оценок хаусдорфовой и фрактальной размерностей множества $\mathfrak{M}\left(\operatorname{dim}_{H} \mathfrak{M}\right.$ и $\operatorname{dim}_{f} \mathfrak{M}$ соответственно). В настоящей работе для случая периодических краевых условий мы устанавливаем ряд новых результатов по регулярности решений задачи $\left(1.1_{k}\right), k=1,2, \ldots, 6$, и доказываем конечномерность аттрактора $\mathfrak{M}$ двумя способами. Первьй из них проще, но оценки для $\operatorname{dim}_{H} \mathfrak{M}$ и $\operatorname{dim}_{f} \mathfrak{M}$ получаются хуже. Второй способ, описанньй в [6], дает более точные оценки. Но для обоснования его применимости мы должны доказывать "равномерную дифференцируемость" разрешающих операторов на аттракторе $\mathfrak{M}$, что требует большей гладкости траекторий на $\mathfrak{M}$ по сравнению с первым способом.

Сформулируем основные результаты данной работы, вводя следующие обозначения:

$$
I=] 0, T\left[, \quad I_{\delta}=\right] \delta, T\left[, \quad Q_{\delta, T}=\Omega \times I_{\delta}, \quad \delta \in\right] 0, T[.
$$

Теорема 1.3. Пусть выполнены все условия теоремы 1.1, и пусть дополнительно

$$
L \equiv \sup _{\varepsilon \in \mathbb{M}}\left|\frac{\partial^{2} D}{\partial \varepsilon^{2}}(\varepsilon)\right|=\sup _{\varepsilon \in \mathbb{M}} \sqrt{\frac{\partial^{2} D}{\partial \varepsilon_{i j} \partial \varepsilon_{k m}}(\varepsilon) \frac{\partial^{2} D}{\partial \varepsilon_{i j} \partial \varepsilon_{k m}}(\varepsilon)}<+\infty .
$$

Тогда $t \partial_{t^{2}} v \in L_{2}\left(I ; H^{-1}\right)$ и верна оценка

$$
\left\|t \partial_{t^{2}} v\right\|_{L_{2}\left(I ; H^{-1}\right)} \leqslant \Phi_{3}\left(T,\|\varphi\|,\|g\|_{L_{2}\left(I ; H^{-1}\right)},\left\|t \partial_{t} g\right\|_{L_{2}\left(I ; H^{-1}\right)}\right) .
$$

Более того, если

$$
\sqrt{t} g \in L_{2}\left(Q_{T} ; \mathbb{R}^{2}\right)
$$

mо $\sqrt{t} \nabla^{2} v \in L_{2}\left(Q_{T} ; \mathbb{R}^{8}\right)$, причем

$$
\left\|\sqrt{t} \nabla^{2} v\right\|_{Q_{T}} \leqslant \Phi_{4}\left(T ;\|\varphi\|,\|g\|_{L_{2}\left(I ; H^{-1}\right)},\left\|t \partial_{t} g\right\|_{L_{2}\left(I ; H^{-1}\right)},\|\sqrt{t} g\|_{Q_{T}}\right) .
$$


ЗАмЕЧАНИЕ 1.1. Из утверждений теорем 1.1 и 1.3 , в частности, следует, что $t \partial_{t} v \in C([0, T] ; H)$ и $t \nabla v \in C\left([0, T] ; L_{2}\left(\Omega ; \mathbb{R}^{4}\right)\right)$.

Следующее утверждение является ключевым в первом способе оценивания $\operatorname{dim}_{H} \mathfrak{M}_{\text {и }} \operatorname{dim}_{f} \mathfrak{M}$.

ТеОрема 1.4. Пусть выполнены условия $\left(1.2_{k}\right), \quad k=1,2,3,(1.8),(1.9), u$ пусть функция $u \in H^{1}$ удовлетворяет вариационному тождеству

$$
\left(\frac{\partial D}{\partial \varepsilon}(\varepsilon(u)), \varepsilon(w)\right)=(f, w) \quad \forall w \in H^{1} .
$$

Тогда если $f \in L_{2}\left(\Omega ; \mathbb{R}^{2}\right)$, то $и \in H^{2}$ и верна оценка

$$
\left\|\nabla^{2} u\right\| \leqslant C_{0}\|f\| \text {. }
$$

Более того, если

$$
f \in L_{2+\alpha_{0}}\left(\Omega ; \mathbb{R}^{2}\right) \quad \text { npu } \quad \alpha_{0}>0
$$

то существует число $\left.\left.\alpha_{1} \in\right] 0, \alpha_{0}\right]$, не зависящее от $f$, такое, что $и \in W_{2+\alpha_{1}}^{2}$ $\left(\Omega ; \mathbb{R}^{2}\right)$ и верна оценка

$$
\left\|\nabla^{2} u\right\|_{2+\alpha_{1}} \leqslant C_{0}^{\prime}\|f\|_{2+\alpha_{1}} .
$$

Теорема 1.4 позволяет доказать следуюшую лемму.

ЛЕмма 1.1. Пусть выполнены условия $\left(1.2_{k}\right), k=1,2,3,(1.9),(1.8),(1.15)$, и пусть дополнительно

$$
L_{1}=\sup _{\varepsilon \in \mathbb{M}}\left|\frac{\partial^{3} D}{\partial \varepsilon^{3}}(\varepsilon)\right|<+\infty
$$

Тогда существует число $m>0$ такое, что

$$
\left\|V_{1}\left(\varphi^{1}\right)-V_{1}(\varphi)\right\|_{(1)} \leqslant m\left\|\varphi^{1}-\varphi\right\| \quad \forall \varphi^{1}, \varphi \in \mathfrak{M} .
$$

Применяя теорему 1 из [9], получаем следующие оценки $\operatorname{dim}_{H} \mathfrak{M}_{\text {и }} \operatorname{dim}_{f} \mathfrak{M}$.

ТеОрема 1.5. Пусть выполнены все условия леммы 1.1. Тогда

$$
\operatorname{dim}_{H} \mathfrak{M} \leqslant \operatorname{dim}_{f} \mathfrak{M} \leqslant N \frac{\ln \left(\frac{8 \varkappa^{2} l^{2}}{1-\delta^{2}}\right)}{\ln \left(\frac{2}{1+\delta^{2}}\right)},
$$

где $N$ выбрано так, чтобы $\delta \equiv \frac{m}{\sqrt{\lambda_{N+1}}}<1, \quad l=\frac{m}{\sqrt{\lambda_{1}}}, a \varkappa-$ постоянная Гаyсcа.

Перейдем к описанию результатов по регулярности решения задачи $\left(1.1_{k}\right)$, $k=1,2, \ldots, 6$, достаточных для доказательства конечномерности аттрактора $\mathfrak{M}$ вторым способом. 
ТЕОРема 1.6. Пусть выполнены все условия теорем 1.1 и 1.3, и пусть дополнительно

$$
t^{\frac{5}{2}} \partial_{t} g \in L_{2}\left(Q_{T} ; \mathbb{R}^{2}\right) \text {. }
$$

Тогда

$$
\begin{aligned}
t^{\frac{5}{2}} \partial_{t} v \ln \left(2+\left|\partial_{t} v\right|^{2}\right) & \in L_{\infty}\left(I ; L_{2}\left(\Omega ; \mathbb{R}^{2}\right)\right) \\
t^{\frac{5}{2}} \partial_{t} v \ln \left(2+\left|\partial_{t} v\right|^{2}\right) & \in L_{2}\left(Q_{T} ; \mathbb{R}^{4}\right)
\end{aligned}
$$

и верна оченка

$$
\begin{aligned}
& \underset{0 \leqslant t \leqslant T}{\operatorname{ess} \sup }\left\|t^{\frac{5}{2}} \partial_{t} v(t) \ln \left(2+\left|\partial_{t} v(t)\right|^{2}\right)\right\|^{2}+\left\|t^{\frac{5}{2}} \nabla \partial_{t} v \ln \left(2+\left|\partial_{t} v\right|^{2}\right)\right\|_{Q_{T}}^{2} \\
& \quad \leqslant \Phi_{5}\left(T,\|\varphi\|,\|g\|_{L_{2}\left(I ; H^{-1}\right)},\left\|t \partial_{t} g\right\|_{L_{2}\left(I ; H^{-1}\right)},\left\|t^{\frac{1}{2}} g\right\|_{Q_{T}},\left\|t^{\frac{5}{2}} \partial_{t} g\right\|_{Q_{T}}\right) .
\end{aligned}
$$

Здесь и всюду в дальнейшем мы будем считать, что все наши функции периодически продолжены на все пространство $\mathbb{R}^{2}$. Для произвольных точки $x_{0} \in \mathbb{R}^{2}$ и числа $R \in] 0,1[$ мы полагаем

$$
\omega\left(x_{0}, R\right)=\left\{x \in \mathbb{R}^{2}:\left|x_{k}-x_{0 k}\right|<R, \quad k=1,2\right\} .
$$

СлЕДСТВИЕ 1.1 (локальный вариант теоремы 1.6). В условиях теоремы 1.6 имеет место следующая оценка:

$$
\underset{0 \leqslant t \leqslant T}{\operatorname{ess} \sup }\left\|t^{\frac{5}{2}} \partial_{t} v(t)\right\|_{\omega\left(x_{0}, R\right)}^{2} \leqslant \frac{1}{\ln ^{2} \frac{1}{R}} \Phi_{6}, \quad 0<R \leqslant \frac{1}{2},
$$

в которой мажсоранта $\Phi_{6}$ зависит от тех же арғументов, что и $\Phi_{5}$.

Оценка типа (1.23) справедлива и для $\nabla^{2} v$.

ТЕОРема 1.7. Пусть выполнены все условия теоремы 1.6, и пусть дополнительно

$$
t^{\frac{5}{2}} g \in L_{\infty}\left(I ; L_{2+\gamma_{0}}\left(\Omega ; \mathbb{R}^{2}\right)\right), \quad \gamma_{0}>0 .
$$

Тогда

$$
\begin{aligned}
\underset{0 \leqslant t \leqslant T}{\operatorname{ess} \sup }\left\|t^{\frac{5}{2}} \nabla^{2} v(t)\right\|_{\omega\left(x_{0}, R\right)}^{2} & \leqslant \frac{1}{\ln \frac{1}{R}} \Phi_{7}\left(T,\|\varphi\|,\|g\|_{L_{2}\left(I ; H^{-1}\right)},\left\|t \partial_{t} g\right\|_{L_{2}\left(I ; H^{-1}\right)},\right. \\
& \left.\left\|t^{\frac{1}{2}} g\right\|_{Q_{T}},\left\|t^{\frac{5}{2}} \partial_{t} g\right\|_{Q_{T}},\left\|t^{\frac{5}{2}} g\right\|_{L_{\infty}\left(I ; L_{2+\gamma_{0}}\left(\Omega ; \mathbb{R}^{2}\right)\right)}\right)
\end{aligned}
$$

для всех $x_{0} \in \mathbb{R}^{2} u 0<R \leqslant 1 / 4$.

При помоши следствия 1.1 и теоремы 1.7 мы доказываем две оценки, на которых основан второй способ доказательства конечномерности аттрактора $\mathfrak{M}$. 
Теорема 1.8. Пусть выполнены условия теоремы (1.7) и условие (1.17), и пусть дополнительно

$$
\left.g \in L_{2}\left(I_{\delta} ; W_{2, \text { per }}^{1}\left(\Omega ; \mathbb{R}^{2}\right)\right) \quad \forall \delta \in\right] 0, T[.
$$

Тогда для всех $\left.\delta_{1} \in\right] \delta, T[$ миц имеем

$$
\begin{aligned}
& \nabla^{2} v \in L_{\infty}\left(I_{\delta_{1}} ; L_{2}\left(\Omega ; \mathbb{R}^{8}\right)\right), \quad \nabla \partial_{t} v \in L_{\infty}\left(I_{\delta_{1}} ; L_{2}\left(\Omega ; \mathbb{R}^{4}\right)\right), \\
& \nabla^{3} v \in L_{2}\left(Q_{\delta_{1}, T} ; \mathbb{R}^{16}\right), \quad \quad \nabla^{2} \partial_{t} v \in L_{2}\left(Q_{\delta_{1}, T} ; \mathbb{R}^{8}\right) \text {. }
\end{aligned}
$$

Более того, верны две оценки:

$$
\begin{aligned}
\underset{\delta_{1} \leqslant t \leqslant T}{\operatorname{ess} \sup _{1}} & \left\|\nabla^{2} v(t)\right\|^{2}+\left\|\nabla^{3} v\right\|_{Q_{\delta_{1}, T}}^{2} \\
\leqslant & \Phi_{8}\left(T,\|\varphi\|,\|g\|_{L_{2}\left(I ; H^{-1}\right)},\left\|t \partial_{t} g\right\|_{L_{2}\left(I ; H^{-1}\right)},\left\|t^{\frac{1}{2}} g\right\|_{Q_{T}},\left\|t^{\frac{5}{2}} \partial_{t} g\right\|_{Q_{T}},\right. \\
& \left.\left\|t^{\frac{5}{2}} g\right\|_{L_{\infty}\left(I ; L_{2+\gamma_{0}}\left(\Omega ; \mathbb{R}^{2}\right)\right)},\|\operatorname{curl} g\|_{Q_{\delta}, T}, \frac{1}{\delta_{1}-\delta}, \frac{1}{\delta}\right)
\end{aligned}
$$

$u$

$$
\underset{\delta_{1} \leqslant t \leqslant T}{\operatorname{ess} \sup }\left\|\nabla \partial_{t} v(t)\right\|^{2}+\left\|\nabla^{2} \partial_{t} v\right\|_{Q_{\delta_{1}, T}}^{2} \leqslant \Phi_{9}
$$

где $0<\delta<\delta_{1}$ и мажсоранта $\Phi_{9}$ зависит от тех жее аргументов, что и $\Phi_{8}$.

СлЕДСТвИЕ 1.2. В условиях теоремы 1.8 верны следующие утверждения:

$$
\begin{gathered}
\nabla^{2} v \in C\left(\bar{I}_{\delta_{1}} ; L^{2}\left(\Omega ; \mathbb{R}^{8}\right)\right), \\
\|\nabla v\|_{W_{4}^{1}\left(Q_{\delta_{1}, T} ; \mathbb{R}^{4}\right)} \leqslant \Phi_{10}, \\
\|v\|_{C^{1}\left(\bar{Q}_{\delta_{1}, T} ; \mathbb{R}^{2}\right)} \leqslant \Phi_{11}
\end{gathered}
$$

для всех $0<\delta<\delta_{1}$, а мажсоранты $\Phi_{10}, \Phi_{11}$ зависят от тех же аргументов, что $и \Phi_{8}$.

Следующее утверждение, дополняющее теорему 1.2 , непосредственно вытекает из полугрупповых свойств семейства разрешаюших операторов $V_{t}$ и теоремы 1.8 .

ТеОрема 1.9. Пусть выполнены условия $\left(1.2_{k}\right), k=1,2,3,4,(1.9),(1.17)$, (1.8), и пусть дополнительно

$$
f \in W_{2, \text { per }}^{1}\left(\Omega ; \mathbb{R}^{2}\right) .
$$


Тогда аттрактор $\mathfrak{M}$ полугруппы $\left\{V_{t}, t \in \mathbb{R}_{+}, H\right\}$ ограничен в $H^{2} \cap C^{1}\left(\bar{\Omega} ; \mathbb{R}^{2}\right)$ и верна оченка

$$
\begin{array}{r}
M_{2}=\sup _{\varphi \in \mathfrak{M}} \sup _{t \in \mathbb{R}^{1}} \int_{t}^{t+1}\left[\left\|\nabla^{2} V_{\theta}(\varphi)\right\|_{4}^{4}+\left\|\nabla \partial_{\theta} V_{\theta}(\varphi)\right\|_{4}^{4}\right. \\
\left.+\left\|\nabla^{3} V_{\theta}(\varphi)\right\|^{2}+\left\|\nabla^{2} \partial_{\theta} V_{\theta}(\varphi)\right\|^{2}\right] d \theta<+\infty .
\end{array}
$$

Пусть $\psi \in H, v(t)=V_{t}(\varphi), t \geqslant 0, \varphi \in H$. Рассмотрим следующую начально-краевую задачу:

$$
\begin{aligned}
& \left\{\begin{array}{l}
\partial_{t} u+(\nabla u) v+(\nabla v) u-\operatorname{div}\left(\frac{\partial^{2} D}{\partial \varepsilon^{2}}(\varepsilon(v)) \varepsilon(u)\right)=0, \\
\operatorname{div} u=0,
\end{array}\right. \\
& \left.u\right|_{t=0}=\psi, \\
& \left\{\begin{array}{l}
\left.u\right|_{x_{k}=-1}=\left.u\right|_{x_{k}=1},\left.\quad \nabla u\right|_{x_{k}=-1}=\left.\nabla u\right|_{x_{k}=1}, \\
\left.p\right|_{x_{k}=-1}=\left.p\right|_{x_{k}=1}, \quad k=1,2 .
\end{array}\right.
\end{aligned}
$$

Уравнения (1.351) получаются в результате формальной линеаризации уравнений $\left(1.1_{k}\right), k=1,2,3,4$, на решении $v$.

Задача $\left(1.35_{k}\right), k=1,2,3$, имеет единственное решение $u \in C(\bar{I} ; H) \cap L_{2}\left(I ; H^{1}\right)$ с $\left.\partial_{t} u \in L_{2}\left(I ; H^{-1}\right), I=\right] 0, T\left[\forall T \in \mathbb{R}_{+}^{1}\right.$, которое удовлетворяет вариационному тождеству

$$
\left(\partial_{t} u(t), w\right)+(\nabla u(t) v(t)+\nabla v(t) u(t), w)+\left(\frac{\partial^{2} D}{\partial \varepsilon^{2}}(\varepsilon(v(t))) \varepsilon(u(t)), \varepsilon(w)\right)=0
$$

$\forall w \in H^{1}$ для п.в. $t \in[0, T]$. Это доказывается теми же методами, что и однозначная глобальная разрешимость задачи $\left(1.1_{k}\right), k=1,2, \ldots, 6$. Обозначим через $\left\{V_{t}^{\prime}(\varphi)\right\}_{t \geqslant 0}$ семейство линейных разрешаюших операторов, действуюших из $H$ в $H$, полагая $u(t)=V_{t}^{\prime}(\varphi) \psi$. Справедлива следуюшая

ЛЕмма 1.2. Пусть выполнены все условия теоремы 1.9. Тогда семейство линейных операторов $V_{1}^{\prime}(\varphi): H \rightarrow H$ равномерно ограничено на $\mathfrak{M}$ :

$$
\sup _{\varphi \in \mathfrak{M}}\left\|V_{1}^{\prime}(\varphi)\right\|<+\infty
$$

и существуют положительное число $r$ и непрерьвная функиия $h:[0, r] \rightarrow \mathbb{R}_{+}^{1}$ такие, что $h(0)=0$, причем

$$
\left\|V_{1}\left(\varphi^{1}\right)-V_{1}(\varphi)-V_{1}^{\prime}(\varphi)\left(\varphi^{1}-\varphi\right)\right\| \leqslant h\left(\left\|\varphi^{1}-\varphi\right\|\right)\left\|\varphi^{1}-\varphi\right\|
$$

для всех $\varphi, \varphi^{1} \in \mathfrak{M}$, удовлетворяющих условию $\left\|\varphi^{1}-\varphi\right\| \leqslant r$. 
ОПРЕДЕЛЕНИЕ 1.1. Пусть $U: H \rightarrow H$ - непрерывный оператор, имеюший компактное инвариантное многообразие $\mathscr{N}$, т.е. $U(\mathscr{N})=\mathscr{N}$. Мы будем говорить, что оператор равномерно квазидифференцируем на $\mathscr{N}$, если существует семейство линейных операторов $\left\{W_{\varphi}: H \rightarrow H\right\}_{\varphi \in \mathfrak{M}}$, удовлетворяющее требованию

$$
\sup _{\varphi \in \mathfrak{M}}\left\|W_{\varphi}\right\|<+\infty
$$

и существуют положительное число $r$ и непрерьвная функция $h:[0, r] \rightarrow \mathbb{R}_{+}^{1}$ такие, что $h(0)=0$,

$$
\left\|U\left(\varphi^{\prime}\right)-U(\varphi)-W_{\varphi}\left(\varphi^{\prime}-\varphi\right)\right\| \leqslant h\left(\left\|\varphi^{\prime}-\varphi\right\|\right)\left\|\varphi^{\prime}-\varphi\right\|
$$

для всех $\varphi^{\prime}, \varphi \in \mathscr{N}$.

Заметим, что равномерная квазидифференцируемость, вообще говоря, не влечет не только равномерной дифференцируемости оператора $U$ на $\mathscr{N}$, но даже и его поточечной дифференцируемости на $\mathscr{N}$. Условие типа равномерной квазидифференцируемости впервые появилось, во-видимому, в [10] (см. также [11] и [12]).

В работе [6] были получены оценки фрактальной и хаусдорфовой размерностей инвариантного множества $\mathscr{N}$ оператора $U$ в предположении, что $U$ равномерно дифференцируем на $\mathscr{N}$. Однако все рассуждения и оценки в [6] остаются в силе, если условие равномерной дифференцируемости $U$ на $\mathscr{N}$ заменить условием равномерной квазидифференцируемости. Поскольку утверждение леммы 1.2 в точности означает равномерную квазидифференцируемость оператора $V_{1}$ на аттракторе $\mathfrak{M}$ (разумеется, таким свойством обладает любой оператор $V_{t}$ ), то, используя аргументы работы [6], можно доказать следуюшую теорему.

Теорема 1.10. Пусть выполнены все условия теоремы 1.9; тогда

$$
\operatorname{dim}_{H} \mathfrak{M} \leqslant \operatorname{dim}_{f} \mathfrak{M}<c_{*} \frac{\|f\|_{(-1)}^{2}}{\nu^{4}}
$$

где $c_{*}-$ абсолютная постоянная.

Наша статья организована следуюшим образом. В $\S 2$ собраны вспомогательные утверждения, которые активно используются на протяжении всей статьи. Теорема 1.3 доказывается в $\S 3 ; \S 4$ посвяшен обоснованию первого способа оценки хаусдорфовой и фрактальной размерностей аттрактора $\mathfrak{M}$. Для того чтобы показать идею второго способа доказательства конечномерности аттрактора $\mathfrak{M}$, мы даем в $§ 5$ и $§ 7$ формальные доказательства оценок теорем 1.5 и 1.8, предполагая решение достаточно гладким. Их корректные доказательства читатель найдет в $\S 9$ и $\S 11$ соответственно. В $\S 6$ найдены мажоранты хаусдорфовой и фрактальной размерностей аттрактора $\mathfrak{M}$ вторым способом и, в частности, доказываются теоремы 1.9 и 1.10. Наконец, $\S 10$ содержит исследование регуляризованной задачи, используемой при доказательстве теоремы 1.8 . 


\section{§2. Вспомогательные соглашения, утверждения, неравенства и тождества}

Всюду в дальнейшем постоянные, зависящие только от $\nu, \nu_{1}, L, L_{1}, \gamma_{0}, \alpha_{0}$, будут обозначаться символами $C, C_{1}$ и т.д., причем в разных неравенствах они могут иметь разные значения.

Начнем с формулировки известных утверждений относительно плотности гладких функций в пространствах, введенных в $§ 1$.

ЛЕмма 2.1. Имеют место следующие утверждения:

(i) $W_{2, \mathrm{per}}^{1}\left(\Omega ; \mathbb{R}^{m}\right)$ есть замыкание в $W_{2}^{1}\left(\Omega ; \mathbb{R}^{m}\right)$ множества ограничений на $\Omega$ бесконечно дифференцируемых функций $f: \mathbb{R}^{2} \rightarrow \mathbb{R}^{m}$, удовлетворяющих условию периодичности

$$
f(x+2 z)=f(x) \quad \forall x \in \Omega, \quad \forall z \in \mathbb{Z}^{2} ;
$$

(ii) $H^{1}=\left\{v \in \stackrel{\circ}{W} \underset{2, \operatorname{per}}{1}\left(\Omega ; \mathbb{R}^{2}\right): \operatorname{div} v=0\right.$ в $\left.\Omega\right\}$;

(iii) $H^{2}=\left\{v \in \stackrel{\circ}{W_{2, \text { per }}^{1}}\left(\Omega ; \mathbb{R}^{2}\right): \nabla v \in \stackrel{\circ}{W_{2, \text { per }}^{1}}\left(\Omega ; \mathbb{R}^{4}\right), \quad \operatorname{div} v=\begin{array}{l}0 \\ \text { в } \Omega\end{array}\right\}$.

При помоши леммы 2.1 и интегрирования по частям устанавливаются следуюшие тождества:

$$
\begin{gathered}
\|\varepsilon(u)\|^{2}=\frac{1}{2}\left(\|\nabla u\|^{2}+\|\operatorname{div} u\|^{2}\right) \quad \forall u \in W_{2, \mathrm{per}}^{1}\left(\Omega ; \mathbb{R}^{2}\right), \\
\|\operatorname{curl} u\|^{2}+\|\operatorname{div} u\|^{2}=\|\nabla u\|^{2} \quad \forall u \in W_{2, \mathrm{per}}^{1}\left(\Omega ; \mathbb{R}^{2}\right),
\end{gathered}
$$

где $\operatorname{curl} u=u_{2,1}-u_{1,2}$,

$$
\begin{gathered}
(\operatorname{div} \sigma, \Delta u)=\left(\sigma_{, k}, \varepsilon(u, k)\right) \quad \forall \sigma \in W_{2, \text { per }}^{1}(\Omega ; \mathbb{M}), \\
\forall u \in W_{2, \text { per }}^{1}\left(\Omega ; \mathbb{R}^{2}\right), \quad \nabla u \in W_{2, \text { per }}^{1}\left(\Omega ; \mathbb{R}^{4}\right) .
\end{gathered}
$$

Отметим еще одно тождество:

$$
\begin{gathered}
\operatorname{curl}^{*}(\varphi \operatorname{curl} u)=-\varphi \Delta u+\operatorname{curl}^{*} \varphi \operatorname{curl} u \\
\forall \varphi \in C^{1}(\Omega), \quad \forall u \in W_{2}^{2}\left(\Omega ; \mathbb{R}^{2}\right),
\end{gathered}
$$

где $\operatorname{curl}^{*} \psi=(\psi, 2,-\psi, 1)$.

В дальнейшем мы будем часто использовать два неравенства: неравенство Пуанкаре

$$
\|u\| \leqslant C\|\nabla u\| \quad \forall u \in \stackrel{\circ}{W_{2, p e r}^{1}}\left(\Omega ; \mathbb{R}^{2}\right)
$$

и неравенство

$$
\|u\|_{4}^{2} \leqslant C\|u\|\|\nabla u\| \quad \forall u \in \stackrel{\circ}{W_{2, p e r}^{1}}\left(\Omega ; \mathbb{R}^{2}\right),
$$


по существу доказанное в [13] (см. также [14]-[16]).

И, наконец, еше одна пара тождеств устанавливается интегрированием по часTЯM:

$(\operatorname{div} \sigma, \varphi \Delta u)_{\omega}=\left(\sigma_{, k}, \varphi \varepsilon\left(u_{, k}\right)\right)_{\omega}+\left(\sigma_{, k}, u_{, k} \odot \nabla \varphi\right)_{\omega}-\left(\operatorname{div} \sigma, u_{, k} \varphi, k\right)_{\omega}$

и

$$
(\operatorname{div} \sigma, \varphi \Delta u)_{\omega}=\left(\sigma_{, k}, \varphi \varepsilon\left(u_{, k}\right)\right)_{\omega}+\left(\sigma, \varepsilon\left(u_{, k}\right) \varphi, k\right)_{\omega}-(\sigma \nabla \varphi, \Delta u) \omega .
$$

Они верны для всех $\varphi \in C_{0}^{1}(\omega), \sigma \in W_{2, \text { loc }}^{1}(\omega ; \mathbb{M})$ и $u \in W_{2, \operatorname{loc}}^{2}\left(\omega ; \mathbb{R}^{2}\right)$.

Сделаем еше несколько замечаний, касаюшихся пространств $H^{k}$. Во-первых, все собственные функции задачи (1.3) принадлежат множеству $\hat{J}^{\infty}$. Во-вторых, мы отождествляем элементы пространств $H^{k}$ с рядами Фурье по собственным функциям задачи (1.3), а именно

$$
\begin{gathered}
f \in H^{k} \Leftrightarrow f=\sum_{i=1}^{\infty}\left(f, \varphi^{i}\right) \varphi^{i}, \\
\|f\|_{(k)}=\left(\sum_{i=1}^{\infty}\left|\left(f, \varphi^{i}\right)\right|^{2} \lambda_{i}^{k}\right)^{\frac{1}{2}}, \quad k=-1,0,1,2, \quad H^{0} \equiv H .
\end{gathered}
$$

В-третьих, легко видеть, что справедливы следующие утверждения:

$$
H=L_{2}\left(\Omega ; \mathbb{R}^{2}\right) \cap H^{-1}, \quad H^{1}=W_{2, \text { per }}^{1}\left(\Omega ; \mathbb{R}^{2}\right) \cap H^{-1} .
$$

\section{§3. Доказательство теоремы 1.3}

Доказательство теоремы 1.3 есть прямое продолжение доказательства теоремы 1 из [5]. Действительно, рассмотрим галёркинские аппроксимации решения начально-краевой задачи $\left(1.1_{k}\right), k=1,2, \ldots, 6$. Они имеют вид

$$
v^{n}(x, t)=\sum_{k=1}^{n} a_{k}^{n}(t) \varphi^{k}(x),
$$

где $\left\{\varphi^{k}\right\}_{k=1}^{n}$ - первые $n$ собственных функций задачи (1.3), а коэффициенты $a_{k}^{n}(t)$ определяются в результате решения следующей системы обыкновенных дифференциальных уравнений:

$$
\begin{aligned}
& \left(\partial_{t} v^{k}(t), w^{n}\right)+\left(\nabla v^{n}(t) v^{k}(t), w^{n}\right) \\
& \quad+\left(\frac{\partial D}{\partial \varepsilon}\left(\varepsilon\left(v^{n}(t)\right)\right), \varepsilon\left(w^{n}\right)\right)=\left(g(t), w^{n}\right) \quad \forall w^{n} \in H_{n}^{1},
\end{aligned}
$$

дополненной начальным условием

$$
v^{n}(0)=P_{n} \varphi=\sum_{k=1}^{n}\left(\varphi, \varphi^{k}\right) \varphi^{k}
$$


Здесь $H_{n}^{1}=\operatorname{Span}\left\{\varphi^{1}, \varphi^{2}, \ldots, \varphi^{n}\right\}, P_{n}$ - ортопроектор из $H$ в $H_{n}^{1}$. Как показано в [5], для $v^{n}$ верны оценки (1.5), (1.7) теоремы 1.1.

Итак, пусть выполнено условие (1.9). Имеем для всякого $w \in H^{1}$

$$
\begin{aligned}
\left(\partial_{t^{2}} v^{n}(t), w\right)= & \left(\partial_{t^{2}} v^{n}, P_{n} w\right)=\left(\partial_{t} v_{i}^{n}(t) v^{n}(t)+v_{i}^{n}(t) \partial_{t} v^{n}(t),\left(P_{n} w\right)_{, i}\right) \\
& -\left(\partial_{t} \sigma^{n}(t), \varepsilon\left(P_{n} w\right)\right)+\left(\partial_{t} g(t), P_{n} w\right),
\end{aligned}
$$

где $\sigma^{n}(t)=\frac{\partial D}{\partial \varepsilon}\left(\varepsilon\left(v^{n}(t)\right)\right)$. Отметим следующие неравенства:

$$
\begin{aligned}
\left|\partial_{t} \sigma^{n}(t)\right|= & \left|\frac{\partial^{2} D}{\partial \varepsilon^{2}}\left(\varepsilon\left(v^{n}(t)\right)\right) \varepsilon\left(\partial_{t} v^{n}(t)\right)\right| \leqslant C\left|\nabla \partial_{t} v^{n}(t)\right|, \\
& \left\|P_{n} w\right\|_{(1)} \leqslant\|w\|_{(1)} \quad \forall w \in H^{1} .
\end{aligned}
$$

Таким образом, три последних соотношения, неравенство (2.6) и (1.5) дают оценку

$$
\begin{aligned}
\left|\left(\partial_{t^{2}} v^{n}(t), w\right)\right| \leqslant C_{1}\left(\left\|\nabla \partial_{t} v^{n}(t)\right\|+\left\|\partial_{t} g(t)\right\|_{(-1)}+\left\|v^{n}(t)\right\|_{4}\left\|\partial_{t} v^{n}(t)\right\|_{4}\right)\|\nabla w\| \\
\leqslant C_{2}\left(\left\|\nabla \partial_{t} v^{n}(t)\right\|+\left\|\partial_{t} g(t)\right\|_{(-1)}\right. \\
\left.\quad+\left\|v^{n}(t)\right\|^{\frac{1}{2}}\left\|\nabla v^{n}(t)\right\|^{\frac{1}{2}}\left\|\partial_{t} v^{n}(t)\right\|^{\frac{1}{2}}\left\|\nabla \partial_{t} v^{n}(t)\right\|^{\frac{1}{2}}\right)\|\nabla w\| \\
\leqslant C_{3}\left(\left\|\nabla \partial_{t} v^{n}(t)\right\|+\left\|\partial_{t} g(t)\right\|_{(-1)}^{2}+\Phi_{1}^{\frac{1}{2}}\left\|\nabla v^{n}(t)\right\|\left\|\partial_{t} v^{n}(t)\right\|\right)\|\nabla w\| .
\end{aligned}
$$

После умножения последнего неравенства на $t$ и учета (1.7) будем иметь

$$
t \|\left(\partial_{t^{2}} v^{n}(t) \|_{(-1)} \leqslant C\left(t\left\|\nabla \partial_{t} v^{n}(t)\right\|+t\left\|\partial_{t} g(t)\right\|_{(-1)}+\Phi_{1}^{\frac{1}{2}} \Phi_{2}^{\frac{1}{2}} \sqrt{t}\left\|\partial_{t} v^{n}(t)\right\|\right)\right.
$$

Отсюда и из (1.7) мы получаем оценку (1.10) для галёркинских приближений.

Перейдем к доказательству оценки (1.12) для галёркинских приближений, предполагая выполненным условие (1.11). Прежде всего отметим, что в случае периодических краевых условий мы имеем

$$
\Delta v^{n}(t) \in H_{n}^{1}
$$

для всех $t \in[0, T]$ и всех $n$. Это позволяет положить $w^{n}=\Delta v^{n}(t)$ в $\left(3.1_{1}\right)$ и получить

$$
\begin{aligned}
& -\left(\partial_{t} v^{n}(t), \Delta v^{n}(t)\right)+\left(\operatorname{div} \sigma^{n}(t), \Delta v^{n}(t)\right) \\
& \quad=-\left(g(t), \Delta v^{n}(t)\right)-\left(\nabla v^{n}(t) v^{n}(t), \Delta v^{n}(t)\right) .
\end{aligned}
$$


Принимая во внимание тождество (2.3), будем иметь

$$
\begin{aligned}
& \frac{1}{2} \partial_{t}\left\|\nabla v^{n}(t)\right\|^{2}+\left(\sigma_{, k}^{n}(t), \varepsilon\left(v_{, k}^{n}(t)\right)\right) \\
& \quad=\frac{1}{2} \partial_{t}\left\|\nabla v^{n}(t)\right\|^{2}+\left(\frac{\partial^{2} D}{\partial \varepsilon^{2}}\left(\varepsilon\left(v^{n}(t)\right) \varepsilon\left(v_{, k}^{n}(t)\right), \varepsilon\left(v_{, k}^{n}(t)\right)\right)\right. \\
& \leqslant\|g(t)\|\left\|\nabla^{2} v^{n}(t)\right\|+\left\|\nabla v^{n}(t)\right\|_{4}\left\|v^{n}(t)\right\|_{4}\left\|\nabla^{2} v^{n}(t)\right\| .
\end{aligned}
$$

Для оценки левой части последнего неравенства воспользуемся последовательно условием $\left(1.2_{3}\right)$ и тождеством $(2.1)$, полагая $u=v_{, k}^{n}(t)$. Правую часть оценим при помощи неравенства (2.6). В результате будем иметь

$$
\begin{aligned}
& \frac{1}{2} \partial_{t}\left\|\nabla v^{n}(t)\right\|^{2}+\nu\left\|\nabla^{2} v^{n}(t)\right\|^{2} \\
& \quad \leqslant\|g(t)\|\left\|\nabla^{2} v^{n}(t)\right\|+C\left\|v^{n}(t)\right\|^{\frac{1}{2}}\left\|\nabla v^{n}(t)\right\|\left\|\nabla^{2} v^{n}(t)\right\|^{\frac{3}{2}},
\end{aligned}
$$

а после применения неравенства Юнга и оценки (1.5) получим

$$
\partial_{t}\left\|\nabla v^{n}(t)\right\|^{2}+\nu\left\|\nabla^{2} v^{n}(t)\right\|^{2} \leqslant C\left[\|g(t)\|^{2}+\Phi_{1}\left\|\nabla v^{n}(t)\right\|^{4}\right] .
$$

Осталось умножить последнее неравенство на $t$ :

$$
\begin{aligned}
& \partial_{t}\left(t\left\|\nabla v^{n}(t)\right\|^{2}\right)+\nu t\left\|\nabla^{2} v^{n}(t)\right\|^{2} \\
& \quad \leqslant C\left(\left\|\nabla v^{n}(t)\right\|^{2}+t\|g(t)\|^{2}\right)+C \Phi_{1}\left\|\nabla v^{n}(t)\right\|^{2}\left(t\left\|\nabla v^{n}(t)\right\|^{2}\right)
\end{aligned}
$$

и установить

$$
\begin{array}{rl}
\sup _{0 \leqslant t \leqslant T} & t\left\|\nabla v^{n}(t)\right\|^{2} \leqslant C \int_{0}^{T}\left(\left\|\nabla v^{n}(t)\right\|^{2}+t\|g(t)\|^{2}\right) d x \\
& \times \exp \left[C \Phi_{1} \int_{0}^{T}\left\|\nabla^{2} v(t)\right\|^{2} d x\right] \leqslant C\left[\Phi_{1}+\|\sqrt{t} g\|_{Q_{T}}^{2}\right] \exp \left(C \Phi_{1}^{2}\right) .
\end{array}
$$

Из двух последних соотношений получаем оценку (1.12) для галёркинских приближений. Теорема 1.3 доказана.

\section{§4. Первый способ доказательства конечномерности аттрактора}

ДОКАЗАТЕЛЬСТВО ТЕОРЕМЫ 1.4. В силу выпуклости потенциала $D$ не существует двух неравных функций, удовлетворяющих тождеству (1.13) с одной и той же правой частью $f$. Рассмотрим функцию $n$ переменных

$$
F\left(b_{1}, \ldots, b_{n}\right)=\int_{\Omega} D\left(\varepsilon\left(w^{n}\right)\right) d x-\left(f, w^{n}\right),
$$


где

$$
w^{n}=\sum_{k=1}^{n} b_{k} \varphi^{k} \in H_{n}^{1}=\operatorname{Span}\left\{\varphi^{1}, \varphi^{2}, \ldots, \varphi^{n}\right\}
$$

а $\varphi^{k}$ - собственные функции задачи (1.3). В силу условий, наложенных на потенциал $D$, функция $F$ достигает своей нижней точной грани в единственной точке $\left(b_{1}^{n}, \ldots, b_{n}^{n}\right)$. Необходимые условия экстремума влекут тождество

$$
\left(\frac{\partial D}{\partial \varepsilon}\left(\varepsilon\left(u^{n}\right)\right), \varepsilon\left(w^{n}\right)\right)=\left(f, w^{n}\right), \quad w^{n} \in H_{n}^{1}
$$

где $u^{n}=\sum_{k=1}^{n} b_{k}^{n} \varphi^{k}$. Рассмотрим разность (1.13) с $w=w^{n}$ и (4.1), взяв в них $w^{n}=P_{n} u-u^{n}$, где $P_{n} u=\sum_{k=1}^{n}\left(u, \varphi_{k}\right) \varphi^{k}$, и результат запишем в виде

$$
\left(\frac{\partial D}{\partial \varepsilon}(\varepsilon(u))-\frac{\partial D}{\partial \varepsilon}\left(\varepsilon\left(u^{n}\right)\right), \varepsilon\left(u-u^{n}\right)\right)=\left(\frac{\partial D}{\partial \varepsilon}(\varepsilon(u))-\frac{\partial D}{\partial \varepsilon}\left(\varepsilon\left(u^{n}\right)\right), \varepsilon\left(u-P_{n} u\right)\right)
$$

Отсюда в силу $\left(1.2_{3}\right),\left(1.2_{4}\right)$ и $(2.1)$ выводим неравенство

$$
\begin{aligned}
\nu\left\|u^{n}-u\right\|_{(1)}^{2} & =2 \nu\left\|\varepsilon\left(u^{n}-u\right)\right\|^{2} \leqslant\left(\frac{\partial D}{\partial \varepsilon}(\varepsilon(u))-\frac{\partial D}{\partial \varepsilon}\left(\varepsilon\left(u^{n}\right)\right), \varepsilon\left(u-P_{n} u\right)\right) \\
& \leqslant C\left\|u-u^{n}\right\|_{(1)}\left\|u-P_{n} u\right\|_{(1)} .
\end{aligned}
$$

Так как $u \in H^{1}$, то $\left\|u-P_{n} u\right\|_{(1)} \rightarrow 0$ при $n \rightarrow+\infty$ и, следовательно,

$$
u^{n} \rightarrow u \quad \text { в } \quad H^{1}
$$

Пусть $f \in L_{2}\left(\Omega ; \mathbb{R}^{2}\right)$. Как мы уже отмечали, в случае периодических краевых условий $\Delta u^{n} \in H_{n}^{1}$. Полагая $w^{n}=\Delta u^{n}$ в (4.1) и учитывая тождества (2.1) и (2.3), получим

$$
\begin{aligned}
\nu\left\|\nabla^{2} u^{n}\right\|^{2} & =2 \nu\left(\varepsilon\left(u_{, k}^{n}\right), \varepsilon\left(u_{, k}^{n}\right)\right) \leqslant\left(\frac{\partial^{2} D}{\partial \varepsilon^{2}}\left(\varepsilon\left(u^{n}\right)\right) \varepsilon\left(u_{, k}^{n}\right), \varepsilon\left(u_{, k}^{n}\right)\right) \\
& =\left(\left(\frac{\partial D}{\partial \varepsilon}\left(\varepsilon\left(u^{n}\right)\right)\right)_{, k}, \varepsilon\left(u_{, k}^{n}\right)\right)=\left(\operatorname{div} \frac{\partial D}{\partial \varepsilon}\left(\varepsilon\left(u^{n}\right)\right), \Delta u^{n}\right)=-\left(f, \Delta u^{n}\right)
\end{aligned}
$$

и, таким образом,

$$
\left\|\nabla^{2} u^{n}\right\| \leqslant \frac{\|f\|}{\nu}
$$

Отсюда и из (4.2) вытекает оценка (1.14).

Предположим теперь, что выполнено условие (1.15). Продолжим функцию $u$ периодическим образом на все пространство $\mathbb{R}^{2}$. Согласно лемме 2.1 продолженная 
таким образом функция принадлежит классу $W_{2, \text { loc }}^{2}\left(\mathbb{R}^{2} ; \mathbb{R}^{2}\right)$. Для того чтобы использовать локальные пробные функции в тождестве (1.13), поступим следующим образом. Для произвольных $x_{0} \in \mathbb{R}^{2}$ и $\left.R \in\right] 0,1[$ введем следующие обозначения:

$$
\begin{gathered}
\omega\left(x_{0}, R\right)=\left\{x \in \mathbb{R}^{2}:\left|x_{k}-x_{0 k}\right|<R, \quad k=1,2\right\}, \\
\omega^{z}\left(x_{0}, R\right)=w\left(x_{0}, R\right)+2 z, \quad z \in \mathbb{Z}^{2}, \\
\Xi\left(x_{0}, R\right)=\bigcup_{z \in \mathbb{Z}^{2}} \omega^{z}\left(x_{0}, R\right), \\
\Omega\left(x_{0}, R\right)=\Omega \cap \Xi\left(x_{0}, R\right) .
\end{gathered}
$$

Пусть, далее, $\varphi \in C_{0}^{1}\left(\mathbb{R}^{2}\right)$ - срезающая функция, удовлетворяющая условиям

$$
\begin{gathered}
0 \leqslant \varphi \leqslant 1, \quad \text { spt } \varphi \subset \omega\left(x_{0}, R\right), \\
\varphi \equiv 1 \quad \text { в } \quad \omega\left(x_{0}, R / 2\right), \\
|\nabla \varphi| \leqslant \frac{C}{R} .
\end{gathered}
$$

Положим

$$
\bar{u}(x)= \begin{cases}u(x)-(\nabla u)_{x_{0}, R}\left(x-x_{0}-2 z\right), & x \in \omega^{z}\left(x_{0}, R\right), \quad z \in \mathbb{Z}^{2}, \\ 0, & x \in \mathbb{R}^{2} \backslash \Xi\left(x_{0}, R\right),\end{cases}
$$

где

$$
(\nabla u)_{x_{0}, R}=f_{\omega\left(x_{0}, R\right)} \nabla u d x
$$

- среднее значение функции $\nabla u$ на квадрате $\omega\left(x_{0}, R\right)$, и

$$
\widehat{\varphi}(x)= \begin{cases}\varphi\left(x-x_{0}-2 z\right), & x \in \omega^{z}\left(x_{0}, R\right), \quad z \in \mathbb{Z}^{2}, \\ 0, & x \in \mathbb{R}^{2} \backslash \Xi\left(x_{0}, R\right) .\end{cases}
$$

Легко видеть, что

$$
\widehat{w}=\operatorname{curl}^{*}\left(\widehat{\varphi}^{2} \operatorname{curl} \bar{u}\right) \in H
$$

С другой стороны, поскольку $u \in H^{2}$, то из (1.13) следует, что

$$
-(\operatorname{div} \sigma, w)=(f, w) \quad \forall w \in H, \quad \sigma=\frac{\partial D}{\partial \varepsilon}(\varepsilon(u))
$$

Полагая $w=\widehat{w}$ в последнем тождестве, получим (см. (2.4))

$$
\begin{aligned}
-(\operatorname{div} \sigma, \widehat{w}) & =\left(\operatorname{div} \sigma, \varphi^{2} \Delta u-\operatorname{curl}^{*} \varphi^{2} \operatorname{curl} \bar{u}\right)_{\omega\left(x_{0}, R\right)} \\
=(f, \widehat{w}) & =-\left(f, \varphi^{2} \Delta u-\operatorname{curl}^{*} \varphi^{2} \operatorname{curl} \bar{u}\right)_{\omega\left(x_{0}, R\right)} .
\end{aligned}
$$


Принимая во внимание тождество $\left(2.7_{2}\right)$ и свойства $\left(4.4_{1}\right)$, выводим из последнего равенства соотношение

$$
\begin{aligned}
& \int_{\omega\left(x_{0}, R\right)} \varphi^{2} \sigma_{, k}: \varepsilon(u, k) d x \leqslant C\left[\left\|\varphi \nabla^{2} u\right\|_{\omega\left(x_{0}, R\right)} \frac{1}{R}\|\nabla \bar{u}\|_{\omega\left(x_{0}, R\right)}\right. \\
& \left.+\|f\|_{\omega\left(x_{0}, R\right)}\left\|\varphi \nabla^{2} u\right\|_{\omega\left(x_{0}, R\right)}+\|f\|_{\omega\left(x_{0}, R\right)} \frac{1}{R}\|\nabla \bar{u}\|_{\omega\left(x_{0}, R\right)}\right] .
\end{aligned}
$$

$\mathrm{C}$ другой стороны, условие $\left(1.2_{3}\right)$ и интегрирование по частям дают

$$
\begin{aligned}
\int_{\omega\left(x_{0}, R\right)} \varphi^{2} \sigma_{, k}: \varepsilon(u, k) d x & \geqslant 2 \nu \int_{\omega\left(x_{0}, R\right)} \varphi^{2} \varepsilon\left(u_{, k}\right): \varepsilon\left(u_{, k}\right) d x \\
& \geqslant \nu\left\|\varphi \nabla^{2} u\right\|_{\omega\left(x_{0}, R\right)}^{2}-C\left\|\varphi \nabla^{2} u\right\|_{\omega\left(x_{0}, R\right)} \frac{1}{R}\|\nabla \bar{u}\|_{\omega\left(x_{0}, R\right)} .
\end{aligned}
$$

Итак, два последних неравенства влекут оценку

$$
\int_{\omega\left(x_{0}, R\right)} \varphi^{2}\left|\nabla^{2} u\right|^{2} d x \leqslant C\left[\int_{\omega\left(x_{0}, R\right)}|f|^{2} d x+\frac{1}{R^{2}} \int_{\omega\left(x_{0}, R\right)}|\nabla \bar{u}|^{2} d x\right]
$$

для всех $R \in] 0,1\left[\right.$ и всех $x_{0} \in \mathbb{R}^{2}$. Отсюда в силу неравенства Пуанкаре

$$
\left(f_{\omega\left(x_{0}, R\right)}|\nabla \bar{u}|^{2} d x\right)^{\frac{1}{2}} \leqslant C R f_{\omega\left(x_{0}, R\right)}\left|\nabla^{2} u\right| d x .
$$

Мы получаем окончательно соотношение

$$
\left(f_{\omega\left(x_{0}, R / 2\right)}\left|\nabla^{2} u\right|^{2} d x\right)^{\frac{1}{2}} \leqslant C\left[\left(f_{\omega\left(x_{0}, R\right)}|f|^{2} d x\right)^{\frac{1}{2}}+f_{\omega\left(x_{0}, R\right)}|\nabla u| d x\right]
$$

которое является локальным вариантом обратного неравенства Гёльдера. Как известно (см., например, [17]), сушествуют числа $\left.\alpha_{1} \in\right] 0, \alpha_{0}\left[\right.$ и $\left.R_{0} \in\right] 0,1[$, которые не зависят от $f$, такие, что $\nabla^{2} u \in L_{2+\alpha_{1}, \text { loc }}\left(\mathbb{R}^{2} ; \mathbb{R}^{2}\right)$ и имеет место оценка

$$
\begin{aligned}
& \left(f_{\omega\left(x_{0}, R / 2\right)}\left|\nabla^{2} u\right|^{2+\alpha_{1}} d x\right)^{\frac{1}{2+\alpha_{1}}} \\
& \quad \leqslant C\left[\left(f_{\omega\left(x_{0}, R\right)}|f|^{2+\alpha_{1}} d x\right)^{\frac{1}{2+\alpha_{1}}}+\left(f_{\omega\left(x_{0}, R\right)}\left|\nabla^{2} u\right|^{2} d x\right)^{\frac{1}{2}}\right]
\end{aligned}
$$

для всех $x_{0} \in \mathbb{R}^{2}$ и $\left.R \in\right] 0, R_{0}$ ]. Положим $R=R_{0} / 2$ в (4.5) и получим (см. (1.14))

$$
\begin{aligned}
& \left(\int_{\omega\left(x_{0}, R_{0} / 4\right)}\left|\nabla^{2} u\right|^{2+\alpha_{1}} d x\right)^{\frac{1}{2+\alpha_{1}}} \\
& \quad \leqslant C_{1}\left[\left(\int_{\Omega}|f|^{2+\alpha_{1}} d x\right)^{\frac{1}{2+\alpha_{1}}}+R_{0}^{\frac{2}{2+\alpha_{1}}-1}\left(\int_{\Omega}\left|\nabla^{2} u\right|^{2} d x\right)^{\frac{1}{2}}\right] \\
& \quad \leqslant C_{2}\left[\|f\|_{2+\alpha_{1}}+R_{0}^{\frac{\alpha_{1}}{2+\alpha}}\|f\|\right] \leqslant C_{3}\|f\|_{2+\alpha_{1}}
\end{aligned}
$$

для всех $x_{0} \in \mathbb{R}^{2}$. Покрывая квадрат $\Omega$ конечным числом квадратов $\omega\left(x_{0}, R_{0} / 4\right)$, устанавливаем оценку (1.16). Теорема 1.4 доказана.

Отметим полезное следствие теоремы 1.4, которое в дальнейшем будет использовано. 
ЛЕмма 4.1. Пусть выполнены все условия теоремы 1.3; тогда для п.в. $t \in[0, T]$ имеет место оценка

$$
\left\|\nabla^{2} v(t)\right\| \leqslant C\left[\|g(t)\|+\left\|\partial_{t} v(t)\right\|+\|v(t)\|\|\nabla v(t)\|^{2}\right] .
$$

ДокАЗАТЕЛЬСтво. Действительно, согласно теореме 1.4 и (2.6) верна оценка

$$
\begin{aligned}
\left\|\nabla^{2} v(t)\right\| & \leqslant C\left\|g(t)-\partial_{t} v(t)-\nabla v(t) v(t)\right\| \\
& \leqslant C\left[\|g(t)\|+\left\|\partial_{t} v(t)\right\|+\|\nabla v(t)\|_{4}\|v(t)\|_{4}\right] \\
& \leqslant C_{1}\left[\|g(t)\|+\left\|\partial_{t} v(t)\right\|+\|v(t)\|^{\frac{1}{2}}\|\nabla v(t)\|\left\|\nabla^{2} v(t)\right\|^{\frac{1}{2}}\right] .
\end{aligned}
$$

Отсюда и из неравенства Юнга получаем (4.6). Лемма 4.1 доказана.

Перейдем к доказательству конечномерности аттрактора.

ЛЕмма 4.2. Пусть выполнены условия $\left(1.2_{k}\right), k=1,2,3,(1.9),(1.8)$ u (1.15). Тогда существует $\left.\left.\alpha_{1} \in\right] 0, \alpha_{0}\right]$ такое, что

$$
M_{3}=\sup _{\varphi \in \mathfrak{M}} \sup _{t \in \mathbb{R}} \int_{t}^{t+1}\left\|\nabla^{2} V_{\theta}(\varphi)\right\|_{2+\alpha_{1}}^{2 \frac{2+\alpha_{1}}{\alpha_{1}}} d \theta<+\infty .
$$

ДоКАЗАТЕЛЬСТВО. В силу теоремы 1.2 и леммы 4.1 мы имеем

$$
\left\|\nabla^{2} v(t)\right\| \leqslant C\left[\|f\|+M_{1}^{\frac{1}{2}}+R_{0} M_{1}\right] \equiv M_{4} \quad \forall \varphi \in \mathfrak{M}, \quad \forall t \in \mathbb{R}^{1},
$$

где $v(t)=V_{t}(\varphi)$. Теперь оценим $\|\tilde{g}(t)\|_{2+\alpha}$, где

$$
\left.\left.\tilde{g}(t)=f-\partial_{t} v(t)-(\nabla v(t)) v(t), \quad \alpha \in\right] 0, \alpha_{0}\right] .
$$

Имеем

$$
\begin{aligned}
\|\tilde{g}(t)\|_{2+\alpha} & \leqslant\|f\|_{2+\alpha}+\left\|\partial_{t} v(t)\right\|_{2+\alpha}+\|v(t)\|_{\infty}\|\nabla v(t)\|_{2+\alpha} \\
& \leqslant\|f\|_{2+\alpha}+\left\|\partial_{t} v(t)\right\|_{2+\alpha}+C\left\|\nabla^{2} v(t)\right\|^{2} \\
& \leqslant\|f\|_{2+\alpha}+\left\|\partial_{t} v(t)\right\|_{2+\alpha}+C M_{4}^{2} .
\end{aligned}
$$

Далее воспользуемся мультипликативным неравенством (см.[15, гл. II])

$$
\left\|\partial_{t} v(t)\right\|_{2+\alpha} \leqslant C\left\|\partial_{t} v(t)\right\|^{\frac{2}{2+\alpha}}\left\|\nabla \partial_{t} v(t)\right\|^{\frac{\alpha}{2+\alpha}} \leqslant C M_{1}^{\frac{1}{2+\alpha}}\left\|\nabla \partial_{t} v(t)\right\|^{\frac{\alpha}{2+\alpha}} .
$$

Из двух последних неравенств выводим

$$
\left.\left.\|\tilde{g}(t)\|_{2+\alpha}^{2 \frac{\alpha+2}{\alpha}} \leqslant M_{5}\left(\left\|\nabla \partial_{t} v(t)\right\|^{2}+1\right) \quad \forall \alpha \in\right] 0, \alpha_{0}\right] .
$$

Отсюда, в частности, следует, что $\tilde{g}(t) \in L_{2+\alpha_{0}}\left(\Omega ; \mathbb{R}^{2}\right)$ при п.в. всех $t \in[0, T]$. Таким образом, мы можем применить теорему 1.4 и получить

$$
\left\|\nabla^{2} v(t)\right\|_{2+\alpha_{1}}^{2 \frac{\alpha_{1}+2}{\alpha_{1}}} \leqslant C M_{5}\left(\left\|\nabla \partial_{t} v(t)\right\|^{2}+1\right) \quad \text { для п.в. } \quad t \in[0, T] .
$$

Утверждение леммы следует из теоремы 1.2 , если положить $M_{3}=C M_{5}\left(M_{1}+1\right)$. Лемма доказана.

Прежде чем доказывать лемму 1.1, докажем для полноты изложения следующее известное утверждение. 
ЛЕмма 4.3. Пусть выполнены условия $\left(1.2_{k}\right), \quad k=1,2,3,4, u$ (1.8). Тогда имеет место оценка

$$
\left\|V_{t}\left(\varphi^{1}\right)-V_{t}(\varphi)\right\|^{2}+\int_{0}^{t}\left\|V_{\theta}\left(\varphi^{1}\right)-V_{\theta}(\varphi)\right\|_{(1)}^{2} d \theta \leqslant C\left\|\varphi^{1}-\varphi\right\|^{2} e^{C M_{1} t}
$$

для всех $t \geqslant 0, \varphi^{1}, \varphi \in \mathfrak{M}$.

ДокАЗАТЕЛЬСТво. Положим $v^{1}(t)=V_{t}\left(\varphi^{1}\right), v(t)=V_{t}(\varphi), \hat{u}(t)=v^{1}(t)-v(t)$. Имеем

$$
\begin{aligned}
& \frac{1}{2} \partial_{t}\|\hat{u}(t)\|^{2}+\left(v_{k}^{1}(t) v_{, k}^{1}(t)-v_{k}(t) v_{, k}(t), \hat{u}(t)\right) \\
& \quad+\left(\frac{\partial D}{\partial \varepsilon}\left(\varepsilon\left(v^{1}(t)\right)\right)-\frac{\partial D}{\partial \varepsilon}(\varepsilon(v(t))), \varepsilon(\hat{u}(t))\right)=0
\end{aligned}
$$

и, следовательно,

$$
\frac{1}{2} \partial_{t}\|\hat{u}(t)\|^{2}+2 \nu\|\varepsilon(\hat{u}(t))\|^{2} \leqslant\left|\left(\hat{u}_{k}(t) v_{, k}(t), \hat{u}(t)\right)\right|
$$

Воспользуемся тождеством (2.1) и неравенством (2.6). В результате получим

$$
\frac{1}{2} \partial_{t}\|\hat{u}(t)\|^{2}+\nu\|\nabla \hat{u}(t)\|^{2} \leqslant C\|\nabla v(t)\|\|\hat{u}(t)\|\|\nabla \hat{u}(t)\| \leqslant C M_{1}^{\frac{1}{2}}\|\hat{u}(t)\|\|\nabla \hat{u}(t)\|
$$

И

$$
\partial_{t}\|\hat{u}(t)\|^{2}+\nu\|\nabla \hat{u}(t)\|^{2} \leqslant C M_{1}\|\hat{u}(t)\|^{2} .
$$

Отсюда легко выводится (4.8). Лемма доказана.

ДоКАЗАТЕЛЬСТВо ЛЕммЫ 1.1. Используя обозначения леммы 4.2, получим из (4.1) соотношение

$$
\left(\partial_{t} \hat{u}+\left(\nabla v^{1}(t)\right) v^{1}(t)-(\nabla v(t)) v(t)-\operatorname{div}\left(\sigma^{1}(t)-\sigma(t)\right), w\right)=0 \quad \forall w \in H
$$

где

$$
\sigma^{1}(t)=\frac{\partial D}{\partial \varepsilon}\left(\varepsilon\left(v^{1}(t)\right)\right), \quad \sigma(t)=\frac{\partial D}{\partial \varepsilon} \varepsilon(v(t)) .
$$

Мы знаем, что $\hat{u} \in H^{2}$. Но тогда для периодических краевых условий $\Delta \hat{u} \in H$. Следовательно, мы можем положить $w=\Delta \hat{u}(t)$ в (4.9). Воспользуемся теперь тождеством (1.3), и в результате будем иметь

$$
\begin{aligned}
& \frac{1}{2} \partial_{t}\|\nabla \hat{u}(t)\|^{2}+\left(\sigma_{, k}^{1}(t)-\sigma_{, k}(t), \varepsilon\left(\hat{u}_{, k}(t)\right)\right) \leqslant\left|\left((\nabla \hat{u}(t)) v^{1}(t)+(\nabla v(t)) \hat{u}(t), \Delta \hat{u}(t)\right)\right| \\
& \quad \leqslant\left\|v^{1}(t)\right\|_{\infty}\|\nabla \hat{u}(t)\|\|\Delta \hat{u}(t)\|+\|\nabla v(t)\|_{4}\|\hat{u}(t)\|_{4}\|\Delta \hat{u}(t)\| \\
& \quad \leqslant C\left(\left\|\nabla^{2} v^{1}(t)\right\|+\left\|\nabla^{2} v(t)\right\|\right)\|\nabla \hat{u}(t)\|\|\Delta \hat{u}(t)\| \leqslant 2 C M_{4}\|\nabla \hat{u}(t)\|\left\|\nabla^{2} \hat{u}(t)\right\| .
\end{aligned}
$$


Остается оценить снизу второе слагаемое в левой части последнего неравенства. А именно

$$
\begin{aligned}
\left(\sigma_{, k}^{1}(t)\right. & \left.-\sigma_{, k}(t), \varepsilon\left(\hat{u}_{, k}(t)\right)\right)=\left(\frac{\partial^{2} D}{\partial \varepsilon^{2}}\left(\varepsilon\left(v^{1}(t)\right)\right) \varepsilon\left(v_{, k}^{1}(t)\right)\right. \\
& \left.-\frac{\partial^{2} D}{\partial \varepsilon^{2}}(\varepsilon(v(t))) \varepsilon\left(v_{, k}(t)\right), \varepsilon\left(\hat{u}_{, k}(t)\right)\right) \\
= & \left(\frac{\partial^{2} D}{\partial \varepsilon^{2}}\left(\varepsilon\left(v^{1}(t)\right)\right) \varepsilon\left(\hat{u}_{, k}(t)\right), \varepsilon\left(\hat{u}_{, k}(t)\right)\right) \\
& +\left(\left[\frac{\partial^{2} D}{\partial \varepsilon^{2}}\left(\varepsilon\left(v^{1}(t)\right)\right)-\frac{\partial^{2} D}{\partial \varepsilon^{2}}(\varepsilon(v(t)))\right] \varepsilon\left(v_{, k}(t)\right), \varepsilon\left(\hat{u}_{, k}(t)\right)\right) \\
\geqslant & 2 \nu\left(\varepsilon\left(\hat{u}_{, k}(t)\right), \varepsilon\left(\hat{u}_{, k}(t)\right)\right)-C_{1} \int_{\Omega}|\varepsilon(\hat{u}(t))||\nabla \varepsilon(v(t))||\nabla \varepsilon(\hat{u}(t))| \\
\geqslant & \nu\left\|\nabla^{2} \hat{u}(t)\right\|-C_{2} \int_{\Omega}|\nabla \hat{u}(t)|\left|\nabla^{2} v(t)\right|\left|\nabla^{2} \hat{u}(t)\right| d x .
\end{aligned}
$$

Итак, два последних соотношения дают

$$
\begin{aligned}
& \frac{1}{2} \partial_{t}\|\nabla \hat{u}(t)\|^{2}+\nu\left\|\nabla^{2} \hat{u}(t)\right\|^{2} \\
& \quad \leqslant C M_{4}\|\nabla \hat{u}(t)\|\left\|\nabla^{2} \hat{u}(t)\right\|+C\left\|\nabla^{2} \hat{u}(t)\right\|\left(\int_{\Omega}|\nabla \hat{u}(t)|^{2}\left|\nabla^{2} v(t)\right|^{2} d x\right)^{\frac{1}{2}}
\end{aligned}
$$

Для оценки последнего слагаемого в (4.10) воспользуемся числом $\alpha_{1}$ леммы 4.2 и неравенством Гёльдера:

$$
\left(\int_{\Omega}|\nabla \hat{u}(t)|^{2}\left|\nabla^{2} v(t)\right|^{2} d x\right)^{\frac{1}{2}} \leqslant\|\nabla \hat{u}(t)\|_{2 \frac{2+\alpha_{1}}{\alpha_{1}}}\left\|\nabla^{2} v(t)\right\|_{2+\alpha_{1}} .
$$

Первый сомножитель в правой части неравенства (4.11) оценим при помощи мультипликативного неравенства:

$$
\|\nabla \hat{u}(t)\|_{2 \frac{2+\alpha_{1}}{\alpha_{1}}} \leqslant C\|\nabla \hat{u}(t)\|^{\frac{\alpha_{1}}{2+\alpha_{1}}}\left\|\nabla^{2} \hat{u}(t)\right\|^{\frac{2}{2+\alpha_{1}}}
$$

Отсюда, а также из (4.10) и (4.11) вытекает оценка

$$
\begin{aligned}
& \frac{1}{2} \partial_{t}\|\nabla \hat{u}(t)\|^{2}+\nu\left\|\nabla^{2} \hat{u}(t)\right\|^{2} \\
& \quad \leqslant C M_{4}\|\nabla \hat{u}(t)\|\left\|\nabla^{2} \hat{u}(t)\right\|+C\left\|\nabla^{2} \hat{u}(t)\right\|^{1+\frac{2}{2+\alpha_{1}}}\|\nabla \hat{u}(t)\| \frac{\alpha_{1}}{2+\alpha_{1}}\left\|\nabla^{2} v(t)\right\|_{2+\alpha_{1}}
\end{aligned}
$$


Оценивая последнее слагаемое с помощью неравенства Юнга, сделаем такой вывод:

$\frac{1}{2} \partial_{t}\|\nabla \hat{u}(t)\|^{2}+\nu\left\|\nabla^{2} \hat{u}(t)\right\|^{2} \leqslant \frac{\nu}{2}\left\|\nabla^{2} \hat{u}(t)\right\|^{2}+M_{6}\|\nabla \hat{u}(t)\|^{2}\left(\left\|\nabla^{2} v(t)\right\|_{2+\alpha_{1}}^{\frac{2\left(2+\alpha_{1}\right)}{\alpha_{1}}}+1\right)$.

После умножения на $t$ выводим из (4.12) оценку

$$
\partial_{t}\left(t\|\nabla \hat{u}(t)\|^{2}\right) \leqslant\|\nabla \hat{u}(t)\|^{2}+2 M_{6}\left(t\|\nabla \hat{u}(t)\|^{2}\right)\left(\left\|\nabla^{2} v(t)\right\|_{2+\alpha_{1}}^{\frac{2\left(2+\alpha_{1}\right)}{\alpha_{1}}}+1\right)
$$

из которой следует, что

$$
t\|\nabla \hat{u}(t)\|^{2} \leqslant \int_{0}^{t}\|\nabla \hat{u}(\sigma)\|^{2} d \sigma \exp \left\{2 M_{6}\left(\int_{0}^{t}\left\|\nabla^{2} v(\theta)\right\|_{2+\alpha_{1}}^{\frac{2\left(2+\alpha_{1}\right)}{\alpha_{1}}} d \theta+t\right)\right\} .
$$

Принимая во внимание утверждения лемм 4.2 и 4.3 , получим окончательно

$$
\|\nabla \hat{u}(1)\|^{2} \leqslant C\left\|\varphi^{1}-\varphi\right\|^{2} \exp \left\{C M_{1}+2 M_{6}\left(M_{3}+1\right)\right\}
$$

Лемма 1.1 доказана.

ДоКАЗАТЕЛЬСТво ТЕОРЕмЫ 1.5. БЛагодаря (1.18)

$$
\begin{aligned}
\left\|Q_{N}(\hat{u}(1))\right\|^{2} & =\left\|\sum_{k=N+1}^{\infty}\left(\hat{u}(1), \varphi^{k}\right) \varphi^{k}\right\|^{2}=\sum_{k=N+1}^{\infty}\left|\left(\hat{u}(1), \varphi^{(k)}\right)\right|^{2} \\
& \leqslant \frac{1}{\lambda_{N+1}} \sum_{k=N+1}^{\infty} \lambda_{k}\left|\left(\hat{u}(1), \varphi^{k}\right)\right|^{2} \leqslant \frac{1}{\lambda_{N+1}}\|\nabla \hat{u}(1)\|^{2} \leqslant \frac{m^{2}}{\lambda_{N+1}}\|\hat{u}(0)\|^{2}
\end{aligned}
$$

и

$$
\left\|P_{N} \hat{u}(1)\right\|^{2}=\left\|\sum_{k=1}^{N}\left(\hat{u}(1), \varphi^{k}\right) \varphi^{k}\right\|^{2} \leqslant\|\hat{u}(1)\|^{2} \leqslant \frac{1}{\lambda_{1}}\|\nabla \hat{u}(1)\|^{2} \leqslant \frac{m^{2}}{\lambda_{1}}\|\hat{u}(0)\|^{2} .
$$

Применяя теперь теорему 1 из [9], получим оценку (1.19). 


\section{§5. Формальный вывод оценки (1.22) и доказательство следствия 1.1}

Начнем с формального вывода глобальной оценки (1.22), предполагая, что решение задачи $\left(1.1_{k}\right), k=1,2, \ldots, 6$, достаточно гладкое. Аккуратное доказательство всех утверждений теоремы 1.6 читатель найдет в $\S 9$.

Рассмотрим при $t>0$ следуюшую краевую задачу:

$$
\begin{gathered}
\Delta q=\operatorname{div}\left(\partial_{t} v(t) \ln ^{2}\left(2+\left|\partial_{t} v(t)\right|^{2}\right)\right)=4 \ln \left(2+\left|\partial_{t} v(t)\right|^{2}\right) \frac{\partial_{t} v(t) \cdot\left(\nabla \partial_{t} v(t)\right) \partial_{t} v(t)}{2+\left|\partial_{t} v(t)\right|^{2}} \\
\left.q\right|_{x_{k}=-1}=\left.q\right|_{x_{k}=1},\left.\quad \nabla q\right|_{x_{k}=-1}=\left.\nabla q\right|_{x_{k}=1}, \quad k=1,2 .
\end{gathered}
$$

Функция $q$ может быть найдена методом Фурье, и в силу $\left(1.21_{2}\right)$ имеем $q \in W_{2}^{2}(\Omega)$ и верна оценка

$$
\left\|\nabla^{2} q\right\| \leqslant C\left\|\nabla \partial_{t} v(t) \ln \left(2+\left|\partial_{t} v(t)\right|^{2}\right)\right\|, \quad t>0
$$

Но тогда первое уравнение в (5.1) и утверждения леммы 2.1 позволяют утверждать, что

$$
w=\partial_{t} v(t) \ln ^{2}\left(2+\left|\partial_{t} v(t)\right|^{2}\right)-c_{0}(t)-\nabla q \in H^{1}
$$

при п.в. $t \in[0, T]$, где

$$
c_{0}(t)=\frac{1}{|\Omega|} \int_{\Omega} \partial_{t} v(t) \ln ^{2}\left(2+\left|\partial_{t} v(t)\right|^{2}\right) d x
$$

Поэтому в силу уравнения $\left(1.1_{1}\right)$, продифференцированного по $t$, справедливо равенство

$$
\begin{aligned}
\left(\partial_{t^{2}} v(t), w\right)+\left(\partial_{t} \sigma(t), \varepsilon(w)\right)= & -\left(\nabla \partial_{t} v(t) v(t), w\right) \\
& -\left(\nabla v(t) \partial_{t} v(t), w\right)+\left(\partial_{t} g(t), w\right)
\end{aligned}
$$

c $w$ из (5.3). Его мы перепишем в виде

$$
I_{1}+I_{2}=I_{3}+I_{4}+I_{5}
$$

Для $I_{1}$ имеем

$$
\begin{aligned}
I_{1} & =\left(\partial_{t^{2}} v(t), \partial_{t} v(t) \ln ^{2}\left(2+\left|\partial_{t} v(t)\right|^{2}\right)-\left(\partial_{t^{2}} v(t), c_{0}(t)\right)-\left(\partial_{t^{2}} v(t), \nabla q\right)\right) \\
& =\left(\partial_{t^{2}} v(t), \partial_{t} v(t) \ln \left(2+\left|\partial_{t} v(t)\right|^{2}\right)\right)=\frac{1}{2} \int_{\Omega} \ln ^{2}\left(2+\left|\partial_{t} v(t)\right|^{2}\right) \partial_{t}\left(2+\left|\partial_{t} v(t)\right|^{2}\right) \\
& =\frac{1}{2} \partial_{t} \int_{\Omega} H_{\infty}\left(\left|\partial_{t} v(t)\right|^{2}\right) d x
\end{aligned}
$$


где

$$
H_{\infty}(z)=(z+2)\left[\ln ^{2}(z+2)-2 \ln (z+2)+2\right], \quad z \geqslant 0
$$

Мы используем неравенство

$$
H_{\infty}(z) \geqslant \frac{1}{2}(z+2) \ln ^{2}(z+2) \geqslant \frac{1}{2} z \ln ^{2}(z+2), \quad z \geqslant 0
$$

Что касается $I_{2}$, то

$$
I_{2}=\left(\partial_{t} \sigma(t), \varepsilon\left(\partial_{t} v(t) \ln ^{2}\left(2+\left|\partial_{t} v(t)\right|^{2}\right)\right)\right)-\left(\partial_{t} \sigma(t), \nabla^{2} q\right)=I_{2}^{\prime}+I_{2}^{\prime \prime}
$$

причем $I_{2}^{\prime \prime}$ оценивается при помощи (1.9) и (5.2) следующим образом:

$$
\begin{aligned}
\left|I_{2}^{\prime \prime}\right| & \leqslant\left\|\partial_{t} \sigma(t)\right\|\left\|\nabla^{2} q\right\| \leqslant\left\|\frac{\partial^{2} D}{\partial \varepsilon^{2}}(\varepsilon(v(t))) \varepsilon\left(\partial_{t} v(t)\right)\right\|\left\|\nabla^{2} q\right\| \\
& \leqslant C\left\|\nabla \partial_{t} v(t)\right\|\left\|\nabla \partial_{t} v(t) \ln \left(2+\left|\partial_{t} v(t)\right|^{2}\right)\right\| .
\end{aligned}
$$

Для краткости обозначим

$$
\mathscr{L} \equiv \frac{\partial^{2} D}{\partial \varepsilon^{2}}(\varepsilon(v(t))) \Rightarrow \partial_{t} \sigma(t)=\mathscr{L} \varepsilon\left(\partial_{t} v(t)\right)
$$

и заметим еще, что

$$
\nabla(\varphi u)=\varphi \nabla u+u \otimes \nabla \varphi, \quad \varepsilon(\varphi u)=\varphi \varepsilon(u)+u \odot \nabla \varphi
$$

Таким образом,

$$
\begin{aligned}
I_{2}^{\prime}= & \left(\mathscr{L} \varepsilon\left(\partial_{t} v(t)\right), \ln \left(2+\left|\partial_{t} v(t)\right|^{2}\right) \varepsilon\left(\partial_{t} v(t) \ln \left(2+\left|\partial_{t} v(t)\right|^{2}\right)\right)\right. \\
& \left.+\partial_{t} v(t) \ln \left(2+\left|\partial_{t} v(t)\right|^{2}\right) \odot \nabla \ln \left(2+\left|\partial_{t} v(t)\right|^{2}\right)\right) \\
= & \left(\mathscr{L}\left(\ln \left(2+\left|\partial_{t} v(t)\right|^{2}\right) \varepsilon\left(\partial_{t} v(t)\right)\right), \varepsilon\left(\partial_{t} v(t) \ln \left(2+\left|\partial_{t} v(t)\right|^{2}\right)\right)\right. \\
& \left.+\partial_{t} v(t) \odot \nabla \ln \left(2+\left|\partial_{t} v(t)\right|^{2}\right)\right) \\
= & \left(\mathscr{L}\left(\varepsilon\left(\partial_{t} v(t) \ln \left(2+\left|\partial_{t} v(t)\right|^{2}\right)\right)-\partial_{t} v(t) \odot \nabla \ln \left(2+\left|\partial_{t} v(t)\right|^{2}\right)\right)\right. \\
& \left.\quad \varepsilon\left(\partial_{t} v(t) \ln \left(2+\left|\partial_{t} v(t)\right|^{2}\right)\right)+\partial_{t} v(t) \odot \nabla \ln \left(2+\left|\partial_{t} v(t)\right|^{2}\right)\right) \\
= & \left(\mathscr{L} \varepsilon\left(\partial_{t} v(t) \ln \left(2+\left|\partial_{t} v(t)\right|^{2}\right)\right), \varepsilon\left(\partial_{t} v(t) \ln \left(2+\left|\partial_{t} v(t)\right|^{2}\right)\right)\right) \\
& -\left(\mathscr{L} \partial_{t} v(t) \odot \nabla \ln \left(2+\left|\partial_{t} v(t)\right|^{2}\right), \partial_{t} v(t) \odot \nabla \ln \left(2+\left|\partial_{t} v(t)\right|^{2}\right)\right) .
\end{aligned}
$$


Далее, последовательно воспользуемся условиями $\left(1.2_{3}\right),(1.9)$ и неравенством Корна, вытекающим из тождества $(2.1)$, и получим

$$
\begin{aligned}
I_{2}^{\prime} & \geqslant 2 \nu\left\|\varepsilon\left(\partial_{t} v(t) \ln \left(2+\left|\partial_{t} v(t)\right|^{2}\right)\right)\right\|^{2}-C_{1}\left\|\partial_{t} v(t) \odot \nabla \ln \left(2+\left|\partial_{t} v(t)\right|^{2}\right)\right\|^{2} \\
& \geqslant \nu\left\|\nabla\left(\partial_{t} v(t) \ln \left(2+\left|\partial_{t} v(t)\right|^{2}\right)\right)\right\|^{2}-C_{2}\left\|\nabla \partial_{t} v(t)\right\|^{2} \\
& =\nu\left\|\nabla \partial_{t} v(t) \ln \left(2+\left|\partial_{t} v(t)\right|^{2}\right)+\partial_{t} v(t) \otimes \nabla \ln \left(2+\left|\partial_{t} v(t)\right|^{2}\right)\right\|^{2}-C_{2}\left\|\nabla \partial_{t} v(t)\right\|^{2} \\
& \geqslant \frac{\nu}{2}\left\|\nabla \partial_{t} v(t) \ln \left(2+\left|\partial_{t} v(t)\right|^{2}\right)\right\|^{2}-C_{3}\left\|\nabla \partial_{t} v(t)\right\|^{2} .
\end{aligned}
$$

Отсюда и из (5.8), (5.9) выводим

$$
I_{2} \geqslant \frac{\nu}{4}\left\|\nabla \partial_{t} v(t) \ln \left(2+\left|\partial_{t} v(t)\right|^{2}\right)\right\|^{2}-C\left\|\nabla \partial_{t} v(t)\right\|^{2} .
$$

Прежде чем оценивать правую часть в (5.5), заметим, что в силу $(2.6),(1.7)$ и (1.12) мы имеем

$$
\begin{aligned}
& \left\|t \partial_{t} v(t)\right\|_{4, Q_{T}}^{2}+\|\sqrt{t} \nabla v\|_{4, Q_{T}}^{2} \\
& \quad \leqslant C\left[\sup _{0 \leqslant t \leqslant T}\left\|t \partial_{t} v(t)\right\|\left\|t \nabla \partial_{t} v(t)\right\|_{Q_{T}}+\sup _{0 \leqslant t \leqslant T}\|\sqrt{t} \nabla v(t)\|\left\|\sqrt{t} \nabla^{2} v\right\|_{Q_{T}}\right] \\
& \quad \leqslant \Phi_{2}^{\prime}\left(T,\|\varphi\|,\|g\|_{L_{2}\left(I ; H^{-1}\right)},\left\|t \partial_{t} g\right\|_{L_{2}\left(I ; H^{-1}\right)}\right)
\end{aligned}
$$

и, следовательно,

$$
\|t v\|_{\infty, Q_{T}} \leqslant \Phi_{2}^{\prime \prime}\left(T,\|\varphi\|,\|g\|_{L_{2}\left(I ; H^{-1}\right)},\left\|t \partial_{t} g\right\|_{L_{2}\left(I ; H^{-1}\right)}\right) .
$$

Далее, $\left|I_{5}\right| \leqslant\left\|\partial_{t} g(t)\right\|\|w\|$, а поскольку

$$
\begin{aligned}
\|w\|^{2} & \leqslant\|w\|^{2}+\|\nabla q\|^{2}=\left\|\partial_{t} v(t) \ln ^{2}\left(2+\left|\partial_{t} v(t)\right|^{2}\right)-c_{0}(t)\right\|^{2} \\
& \leqslant\left\|\partial_{t} v(t) \ln ^{2}\left(2+\left|\partial_{t} v(t)\right|^{2}\right)\right\|^{2} \leqslant C_{1}\left\|\left|\partial_{t} v(t)\right|\left(1+\left|\partial_{t} v(t)\right|\right)\right\|^{2} \\
& \leqslant C_{2}\left[\left\|\partial_{t} v(t)\right\|^{2}+\left\|\partial_{t} v(t)\right\|_{4}^{4}\right],
\end{aligned}
$$

TO

$$
\left|I_{5}\right| \leqslant C\left\|\partial_{t} g(t)\right\|\left[\left\|\partial_{t} v(t)\right\|^{2}+\left\|\partial_{t} v(t)\right\|_{4}^{4}\right]^{\frac{1}{2}} .
$$

Для двух других интегралов правой части (5.5), используя (5.14), мы получаем

$$
\begin{aligned}
\left|I_{3}\right| & +\left|I_{4}\right| \leqslant\|v(t)\|_{\infty}\left\|\nabla \partial_{t} v(t)\right\|\|w\|+\|w\|\left\|\partial_{t} v(t)\right\|_{4}\|\nabla v(t)\|_{4} \\
& \leqslant C_{1}\left(\left\|\partial_{t} v(t)\right\|^{2}+\left\|\partial_{t} v(t)\right\|_{4}^{4}\right)^{\frac{1}{2}}\left[\left\|\nabla \partial_{t} v(t)\right\|\|v(t)\|_{\infty}+\left\|\partial_{t} v(t)\right\|_{4}\|\nabla v(t)\|_{4}\right] \\
& \leqslant C_{1}\left[\left\|\partial_{t} v(t)\right\|^{2}+\left\|\partial_{t} v(t)\right\|_{4}^{4}+\left\|\nabla \partial_{t} v(t)\right\|^{2}\|v(t)\|_{\infty}^{2}+\left\|\partial_{t} v(t)\right\|_{4}^{2}\|\nabla v(t)\|_{4}^{2}\right] \\
& \leqslant C_{2}\left[\left\|\partial_{t} v(t)\right\|^{2}+\left\|\partial_{t} v(t)\right\|_{4}^{4}+\|\nabla v(t)\|_{4}^{4}+\left\|\nabla \partial_{t} v(t)\right\|^{2}\|v(t)\|_{\infty}^{2}\right] .
\end{aligned}
$$


Собирая оценки (5.16), (5.15), (5.11) и (5.6), выводим из (5.4) и (5.5) неравенство

$$
\begin{aligned}
& \partial_{t} \int_{\Omega} H_{\infty}\left(\left|\partial_{t} v(t)\right|^{2}\right) d x+\frac{\nu}{2}\left\|\nabla \partial_{t} v(t) \ln \left(2+\left|\partial_{t} v(t)\right|^{2}\right)\right\|^{2} \\
& \quad \leqslant C\left[\left\|\partial_{t} g(t)\right\|^{2}+\left(1+\|v(t)\|_{\infty}^{2}\right)\left\|\nabla \partial_{t} v(t)\right\|^{2}+\left\|\partial_{t} v(t)\right\|_{4}^{4}+\|\nabla v(t)\|_{4}^{4}\right] .
\end{aligned}
$$

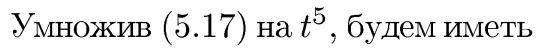

$$
\begin{aligned}
& \partial_{t}\left(t^{5} \int_{\Omega} H_{\infty}\left(\left|\partial_{t} v(t)\right|^{2}\right) d x+\frac{\nu}{2}\left\|t^{\frac{5}{2}} \nabla \partial_{t} v(t) \ln \left(2+\left|\partial_{t} v(t)\right|^{2}\right)\right\|^{2}\right) \\
& \leqslant 5 t^{4} \int_{\Omega} H_{\infty}\left(\left|\partial_{t} v(t)\right|^{2}\right) d x+C\left[\left\|t^{\frac{5}{2}} \partial_{t} g(t)\right\|^{2}+T\left(T^{2}+\|t v\|_{\infty, Q_{T}}^{2}\right)\left\|t \nabla \partial_{t} v(t)\right\|^{2}\right. \\
& \left.\quad+T\left(\left\|t \partial_{t} v(t)\right\|_{4}^{4}+T^{2}\left\|t^{\frac{1}{2}} \nabla v(t)\right\|_{4}^{4}\right)\right] .
\end{aligned}
$$

Принимая во внимание неравенство

$$
H_{\infty}(z) \leqslant C\left(1+z^{2}\right)
$$

и оценки (5.12), (5.13) и (1.12), получаем из (5.18)

$$
\begin{aligned}
& \underset{0 \leqslant t \leqslant T}{\operatorname{ess} \sup _{0}} t^{5} \int_{\Omega} H_{\infty}\left(\left|\partial_{t} v(t)\right|^{2}\right) d x+\frac{\nu}{2}\left\|t^{\frac{5}{2}} \nabla \partial_{t} v(t) \ln \left(2+\left|\partial_{t} v(t)\right|^{2}\right)\right\|_{Q_{T}}^{2} \\
& \leqslant C\left[T^{4}+\left(\Phi_{2}^{\prime}\right)^{2}+\left\|t^{\frac{5}{2}} \partial_{t} g\right\|_{Q_{T}}^{2}+T\left(T^{2}+\left(\Phi_{2}^{\prime \prime}\right)^{2}\right) \Phi_{2}+T\left(1+T^{2}\right)\left(\Phi_{2}^{\prime}\right)^{2}\right] .
\end{aligned}
$$

Отсюда и из (5.7) вытекает оценка (1.22).

ДОКАЗАТЕЛЬСТВО СЛЕДСТВИЯ 1.1. Воспользуемся обозначениями (4.3). В силу неравенства Йенсена мы имеем

$$
\begin{aligned}
& f_{\Omega\left(x_{0}, R\right)}\left|\partial_{t} v(t)\right|^{2} d x \ln ^{2}\left(2+f_{\Omega\left(x_{0}, R\right)}\left|\partial_{t} v(t)\right|^{2} d x\right) \\
& \quad \leqslant f_{\Omega\left(x_{0}, R\right)}\left|\partial_{t} v(t)\right|^{2} \ln ^{2}\left(2+\left|\partial_{t} v(t)\right|^{2}\right) d x \equiv \frac{H(t)}{\left|\Omega\left(x_{0}, R\right)\right|}=\frac{H(t)}{4 R^{2}}
\end{aligned}
$$

где

$$
H(t)=\left\|\partial_{t} v(t) \ln \left(2+\left|\partial_{t} v(t)\right|^{2}\right)\right\|^{2},
$$

а символом

$$
f_{\Omega\left(x_{0}, R\right)} f d x
$$


обозначается среднее значение функции $f$ на множестве $\Omega\left(x_{0}, R\right)$. В силу периодичности получаем из (5.20)

$$
\int_{\omega\left(x_{0}, R\right)}\left|\partial_{t} v(t)\right|^{2} d x \leqslant \frac{H(t)}{\ln ^{2}\left(2+f_{\omega\left(x_{0}, R\right)}\left|\partial_{t} v(t)\right|^{2} d x\right)} .
$$

Возможны два случая:

$$
f_{\omega\left(x_{0}, R\right)}\left|\partial_{t} v(t)\right|^{2} d x \leqslant \frac{2}{R} \Rightarrow \int_{\omega\left(x_{0}, R\right)}\left|\partial_{t} v(t)\right|^{2} d x \leqslant 8 R
$$

И

$$
\begin{aligned}
f_{\omega\left(x_{0}, R\right)}\left|\partial_{t} v(t)\right|^{2} d x & >\frac{2}{R} \stackrel{(5.21)}{\Rightarrow} \int_{\omega\left(x_{0}, R\right)}\left|\partial_{t} v(t)\right|^{2} d x \\
& \leqslant \frac{H(t)}{\ln ^{2}\left(2+\frac{2}{R}\right)}=\frac{H(t)}{\left[\ln \frac{1}{R}+\ln (2+2 R)\right]^{2}} \leqslant \frac{H(t)}{\ln ^{2} \frac{1}{R}}
\end{aligned}
$$

Поэтому верна оценка

$$
\left.\left.\int_{\omega\left(x_{0}, R\right)}\left|\partial_{t} v(t)\right|^{2} d x \leqslant \max \left\{8 R, \frac{H(t)}{\ln ^{2} \frac{1}{R}}\right\} \leqslant C_{*} \max \{1, H(t)\} \frac{1}{\ln ^{2} \frac{1}{R}}, \quad R \in\right] 0, \frac{1}{2}\right],
$$

где $C_{*}=\sup _{0<R \leqslant 1 / 2} 8 R \ln ^{2} \frac{1}{R}$. Если положить $\Phi_{6}=C_{*} \max \left\{T^{5}, \Phi_{5}\right\}$, то из $(1.22)$ вытекает локальная оценка (1.23). Следствие 1.1 доказано.

\section{§6. Доказательство теоремы 1.7}

Утверждение (1.12) теоремы 1.3 позволяет переписать вариационное тождество (1.4) в виде

$$
-(\operatorname{div} \sigma(t), w)=(\bar{g}(t), w) \quad \forall w \in H \quad \text { и п.в. } \quad t \in[0, T],
$$

где

$$
\bar{g}(t)=g(t)-\partial_{t} v(t)-v_{k}(t) v_{, k}(t), \quad \sigma(t)=\frac{\partial D}{\partial \varepsilon}(\varepsilon(v(t))) .
$$

Согласно оценке 4.6 леммы 4.1 мы имеем

$$
\begin{aligned}
\underset{0 \leqslant t \leqslant T}{\operatorname{esssup}}\left\|t^{\frac{5}{2}} \nabla^{2} v(t)\right\|^{2} \leqslant & C\left[\left\|t^{\frac{5}{2}} g\right\|_{L_{\infty}\left(I ; L_{2}\left(\Omega ; \mathbb{R}^{2}\right)\right)}^{2}+T^{3} \sup _{0 \leqslant t \leqslant T}\left\|t \partial_{t} v(t)\right\|^{2}\right. \\
& \left.+T^{4} \sup _{0 \leqslant t \leqslant T}\|v(t)\|^{2} \sup _{0 \leqslant t \leqslant T}\left\|t^{\frac{1}{2}} \nabla v(t)\right\|^{2}\right] .
\end{aligned}
$$


Вспоминая утверждение теоремы 1.1 , получаем

$$
\begin{aligned}
& \operatorname{ess} \sup _{0 \leqslant t \leqslant T}\left\|t^{\frac{5}{2}} \nabla^{2} v(t)\right\|^{2} \leqslant C\left[\left\|t^{\frac{5}{2}} g\right\|_{L_{\infty}\left(I ; L_{2}\left(\Omega ; \mathbb{R}^{2}\right)\right)}^{2}+T^{3} \Phi_{2}+T^{4} \Phi_{1} \Phi_{2}\right] \\
& \quad \leqslant \Phi_{7}^{\prime}\left(T,\|\varphi\|,\|g\|_{L_{2}\left(I ; H^{-1}\right)},\left\|t \partial_{t} g\right\|_{L_{2}\left(I ; H^{-1}\right)},\left\|t^{\frac{1}{2}} g\right\|_{Q_{T}},\left\|t^{\frac{5}{2}} g\right\|_{L_{\infty}\left(I ; L_{2+\gamma_{0}}\left(\Omega ; \mathbb{R}^{2}\right)\right)}\right) .
\end{aligned}
$$

Для построения локальной пробной функции воспользуемся обозначениями (4.3), срезающей функцией $\varphi$, удовлетворяющей условиям $\left(4.4_{1}\right)$, и ее периодическим продолжением $\widehat{\varphi}$, определяемым равенством $\left(4.4_{2}\right)$. Положим

$$
\bar{v}(x, t)= \begin{cases}v(x, t)-[\nabla v(t)]_{x_{0}, R}\left(x-x_{0}-2 z\right), & x \in w^{z}\left(x_{0}, R\right), \quad z \in \mathbb{Z}^{2}, \\ 0, & x \in \mathbb{R}^{2} \backslash \Xi\left(x_{0}, R\right),\end{cases}
$$

где

$$
[\nabla v(t)]_{x_{0}, R}=f_{T\left(x_{0}, R\right)} \nabla v(t) d x
$$

- среднее значение функции $\nabla v(t)$ на множестве

$$
T\left(x_{0}, R\right)=\omega\left(x_{0}, R\right) \backslash \bar{\omega}\left(x_{0}, \frac{R}{2}\right)
$$

Справедливо

$$
\widehat{w}(t)=\operatorname{curl}^{*}\left(\widehat{\varphi}^{2} \operatorname{curl} \bar{v}(t)\right) \in H
$$

при п.в. $t \in[0, T]$. Поэтому мы можем положить $w=\widehat{w}(t)$ в $(6.1)$ и получить

$$
-(\operatorname{div} \sigma(t), \widehat{w}(t))=(\bar{g}(t), \widehat{w}(t)) \quad \text { для п.в. } \quad t \in[0, T] .
$$

Далее, в силу периодичности и тождества (2.4) имеем

$$
\begin{aligned}
-(\operatorname{div} \sigma(t), \widehat{w}(t)) & =\left(\operatorname{div} \sigma(t), \varphi^{2} \Delta v(t)\right)_{\omega\left(x_{0}, R\right)}-\left(\operatorname{div} \sigma(t), \operatorname{curl}^{*} \varphi^{2} \operatorname{curl} \bar{v}(t)\right)_{\omega\left(x_{0}, R\right)} \\
& \geqslant\left(\operatorname{div} \sigma(t), \varphi^{2} \Delta v(t)\right)-\frac{C}{R}\left\|\nabla^{2} v(t)\right\|_{T\left(x_{0}, R\right)}\|\nabla \bar{v}(t)\|_{T\left(x_{0}, R\right)} .
\end{aligned}
$$

Тождество $\left(2.7_{1}\right)$ влечет

$$
\begin{aligned}
-(\operatorname{div} \sigma(t), \widehat{w}(t)) \geqslant & \left(\sigma_{, k}(t), \varphi^{2} \varepsilon\left(v_{, k}(t)\right)\right)_{\omega\left(x_{0}, R\right)} \\
& -\frac{C}{R}\left\|\nabla^{2} v(t)\right\|_{T\left(x_{0}, R\right)}\|\nabla \bar{v}(t)\|_{T\left(x_{0}, R\right)}
\end{aligned}
$$

С другой стороны, условие $\left(1.2_{3}\right)$ и интегрирование по частям дают

$$
\begin{aligned}
& \left(\sigma_{, k}(t), \varphi^{2} \varepsilon\left(v_{, k}(t)\right)\right)_{\omega\left(x_{0}, R\right)} \geqslant 2 \nu\|\varphi \nabla \varepsilon(v(t))\|_{\omega\left(x_{0}, R\right)}^{2} \\
& \geqslant \frac{\nu}{4}\left\|\varphi \nabla^{2} v(t)\right\|_{\omega\left(x_{0}, R\right)}^{2}-\frac{C}{R}\left\|\nabla^{2} v(t)\right\|_{T\left(x_{0}, R\right)}\|\nabla \bar{v}(t)\|_{T\left(x_{0}, R\right)}
\end{aligned}
$$


Из трех последних соотношений вьводим

$$
-(\operatorname{div} \sigma(t), \widehat{w}(t)) \geqslant \frac{\nu}{4}\left\|\varphi \nabla^{2} v(t)\right\|_{\omega\left(x_{0}, R\right)}^{2}-\frac{C}{R}\left\|\nabla^{2} v(t)\right\|_{T\left(x_{0}, R\right)}\|\nabla \bar{v}(t)\|_{T\left(x_{0}, R\right)} .
$$

Для правой части равенства (6.3) имеем

$$
\begin{aligned}
&(\bar{g}(t), \widehat{w}(t))=\left(\bar{g}(t),-\varphi^{2} \Delta v(t)+\operatorname{curl}^{*} \varphi^{2} \operatorname{curl} \bar{v}(t)\right)_{\omega\left(x_{0}, R\right)} \\
& \leqslant\left|\int_{\omega\left(x_{0}, R\right)} v_{k}(t) v_{, k}(t) \cdot \operatorname{curl}^{*}\left(\varphi^{2} \operatorname{curl} \bar{v}(t)\right) d x\right| \\
&+C\left[\|g(t)\|_{\omega\left(x_{0}, R\right)}+\left\|\partial_{t} v(t)\right\|_{\omega\left(x_{0}, R\right)}\right] \\
& \times\left[\left\|\varphi \nabla^{2} v(t)\right\|_{\omega\left(x_{0}, R\right)}+\frac{1}{R}\|\nabla \bar{v}(t)\|_{\omega\left(x_{0}, R\right)}\right] .
\end{aligned}
$$

Заметим еще, что

$$
\begin{aligned}
& \left|\int_{\omega\left(x_{0}, R\right)} v_{k}(t) v_{, k}(t) \cdot \operatorname{curl}^{*}\left(\varphi^{2} \operatorname{curl} v(t)\right) d x\right| \\
& \quad=\left|\int_{\omega\left(x_{0}, R\right)} v_{k}(t) \operatorname{curl} v_{, k}(t) \varphi^{2} \operatorname{curl} v(t) d x\right| \\
& \quad=\left.\left|\int_{T\left(x_{0}, R\right)} v_{k}(t) \varphi \varphi_{, k}\right| \operatorname{curl} \bar{v}(t)\right|^{2} d x \mid \leqslant \frac{C}{R}\|v(t)\|_{T\left(x_{0}, R\right)}\|\nabla \bar{v}(t)\|_{4, T\left(x_{0}, R\right)}^{2} \\
& \quad \leqslant \frac{C}{R}\|v(t)\|\|\nabla \bar{v}(t)\|_{4, T\left(x_{0}, R\right)}^{2} \stackrel{(1.5)}{\leqslant} \frac{C}{R} \Phi_{1}^{\frac{1}{2}}\|\nabla \bar{v}(t)\|_{4, T\left(x_{0}, R\right)}^{2} .
\end{aligned}
$$

Собирая оценки (6.4), (6.5) и применяя неравенство Юнга, выводим из (6.3) оценку

$$
\begin{aligned}
& \left\|\nabla^{2} v(t)\right\|_{\omega\left(x_{0}, R / 2\right)} \leqslant\left\|\varphi \nabla^{2} v(t)\right\|_{\omega\left(x_{0}, R\right)}^{2} \\
& \leqslant C\left[\left\|\nabla^{2} v(t)\right\|_{T\left(x_{0}, R\right)}^{2}+\frac{1}{R^{2}}\|\nabla \bar{v}(t)\|_{T\left(x_{0}, R\right)}^{2}\right. \\
& \left.\quad+\Phi_{1}^{\frac{1}{2}} \frac{1}{R}\|\nabla \bar{v}(t)\|_{4, T\left(x_{0}, R\right)}^{2}+\|g(t)\|_{\omega\left(x_{0}, R\right)}^{2}+\left\|\partial_{t} v(t)\right\|_{\omega\left(x_{0}, R\right)}\right] .
\end{aligned}
$$

Из теорем вложения вытекают два неравенства:

$$
\begin{gathered}
\|\nabla \bar{v}(t)\|_{T\left(x_{0}, R\right)}^{2} \leqslant C R^{2}\left\|\nabla^{2} v(t)\right\|_{T\left(x_{0}, R\right)}^{2}, \\
\|\nabla v(t)\|_{4, T\left(x_{0}, R\right)}^{2} \leqslant C R\left\|\nabla^{2} v(t)\right\|_{T\left(x_{0}, R\right)}^{2}
\end{gathered}
$$

и, следовательно,

$$
\begin{aligned}
\left\|\nabla^{2} v(t)\right\|_{\omega\left(x_{0}, R / 2\right)}^{2} \leqslant & C\left(1+\Phi_{1}^{\frac{1}{2}}\right)\left\|\nabla^{2} v(t)\right\|_{T\left(x_{0}, R\right)}^{2} \\
& +C\left[\|g(t)\|_{\omega\left(x_{0}, R\right)}^{2}+\left\|\partial_{t} v(t)\right\|_{\omega\left(x_{0}, R\right)}^{2}\right] .
\end{aligned}
$$


Поскольку

$$
\left\|\nabla^{2} v(t)\right\|_{T\left(x_{0}, R\right)}^{2}=\left\|\nabla^{2} v(t)\right\|_{\omega\left(x_{0}, R\right)}^{2}-\left\|\nabla^{2} v(t)\right\|_{\omega\left(x_{0}, R / 2\right)}^{2}
$$

то из последнего неравенства можно вывести оценку

$$
\left\|\nabla^{2} v(t)\right\|_{\omega\left(x_{0}, R / 2\right)}^{2} \leqslant \Theta\left\|\nabla^{2} v(t)\right\|_{\omega\left(x_{0}, R\right)}^{2}+C\left[\|g(t)\|_{\omega\left(x_{0}, R\right)}^{2}+\left\|\partial_{t} v(t)\right\|_{\omega\left(x_{0}, R\right)}^{2}\right]
$$

где

$$
0<\frac{C}{C+1} \leqslant \Theta \equiv \frac{C\left(1+\Phi_{1}^{\frac{1}{2}}\right)}{1+C\left(1+\Phi_{1}^{\frac{1}{2}}\right)}<1 .
$$

И, наконец, полагая,

$$
G(R) \equiv \underset{0 \leqslant t \leqslant T}{\operatorname{ess} \sup }\left\|t^{\frac{5}{2}} \nabla^{2} v(t)\right\|_{\omega\left(x_{0}, R\right)}^{2}
$$

получим

$$
G(R / 2) \leqslant \Theta G(R)+C\left[\underset{\substack{\operatorname{ess} \sup \\ 0 \leqslant t \leqslant T}}{5} t^{5}\|g(t)\|_{\omega\left(x_{0}, R\right)}^{2}+\underset{0 \leqslant t \leqslant T}{\operatorname{ess} \sup }\left\|t^{\frac{5}{2}} \partial_{t} v(t)\right\|_{\omega\left(x_{0}, R\right)}^{2}\right] .
$$

Далее, принимая во внимание условие (1.24), будем иметь

$$
\begin{aligned}
t^{5}\|g(t)\|_{\omega\left(x_{0}, R\right)}^{2} & \leqslant t^{5}\left|\omega\left(x_{0}, R\right)\right|^{\frac{2 \gamma_{0}}{2+\gamma_{0}}}\|g(t)\|_{2+\gamma_{0}, \omega\left(x_{0}, R\right)}^{2} \\
& \leqslant C_{1} R^{\frac{4 \gamma_{0}}{2+\gamma_{0}}}\left\|t^{\frac{5}{2}} g(t)\right\|_{2+\gamma_{0}}^{2} \leqslant C_{2} \frac{1}{\ln ^{2} \frac{1}{R}}\left\|t^{\frac{5}{2}} g(t)\right\|_{2+\gamma_{0}}^{2}
\end{aligned}
$$

для $0<R \leqslant 1 / 4$. Последнее слагаемое в правой части (6.8) оценивается при помощи следствия 1.1. В результате получаем неравенство

$$
G(R / 2) \leqslant \Theta G(R)+\frac{1}{\ln ^{2} \frac{1}{R}} \Phi_{7}^{\prime \prime}, \quad 0<R \leqslant \frac{1}{4},
$$

в котором мажоранта $\Phi_{7}^{\prime \prime}$ зависит от тех же аргументов, что и $\Phi_{7}$ в (1.25).

Неравенство (6.9) можно проитерировать:

$$
G\left(\frac{R}{2^{k}}\right) \leqslant \Theta^{k} G(R)+\Phi_{7}^{\prime \prime} \sum_{i=0}^{k-1} \frac{\Theta^{k-1-i}}{\ln ^{2}\left(\frac{2^{i}}{R}\right)}, \quad k=1,2, \ldots
$$

Для того чтобы оценить последнее слагаемое в правой части (6.10), рассмотрим сумму

$$
I(k, \Theta, R) \equiv \Theta^{k} \sum_{i=1}^{k} \frac{\Theta^{-i}}{\left(\ln \frac{1}{R}+i \ln 2\right)^{2}} .
$$


Имеем неравенство

$$
\frac{\Theta^{-i}}{\left(\ln \frac{1}{R}+x \ln 2\right)^{2}} \leqslant \int_{i-1}^{i} \frac{\Theta^{-x-1}}{\left(\ln \frac{1}{R}+x \ln 2\right)^{2}} d x
$$

и, следовательно,

$$
\begin{aligned}
I(k, \Theta, R) & \leqslant \Theta^{k-1} \int_{0}^{k} \frac{\Theta^{-x}}{\left(\ln \frac{1}{R}+x \ln 2\right)^{2}} d x=\Theta^{-1}\left[\Theta^{k} \int_{k / 2}^{k} \cdots+\Theta^{k} \int_{0}^{k / 2} \cdots\right] \\
& \leqslant \Theta^{-1}\left[\int_{k / 2}^{k} \frac{d x}{\left(\ln \frac{1}{R}+x \ln 2\right)^{2}}+\Theta^{\frac{k}{2}} \int_{0}^{k / 2} \frac{d x}{\left(\ln \frac{1}{R}+x \ln 2\right)^{2}}\right] \\
& \leqslant \frac{\Theta^{-1}}{\ln 2}\left[\frac{1}{\ln \frac{1}{R}+\frac{k}{2} \ln 2}-\frac{1}{\ln \frac{1}{R}+k \ln 2}+\frac{\Theta^{\frac{k}{2}}}{\ln \frac{1}{R}}\right] \\
& \leqslant \frac{1}{\Theta \ln 2}\left[\frac{\frac{k}{2} \ln 2}{\left(\ln \frac{1}{R}+\frac{k}{2} \ln 2\right)} \cdot \frac{1}{\left(\ln \frac{1}{R}+k \ln 2\right)}+\frac{\Theta^{\frac{k}{2}}}{\ln \frac{1}{R}}\right] \\
& \leqslant \frac{1}{\Theta \ln 2}\left[\frac{1}{\ln \frac{1}{R}+k \ln 2}+\frac{\Theta^{\frac{k}{2}}}{\ln \frac{1}{R}}\right] \leqslant \frac{1}{\Theta \ln 2}\left[\frac{1}{\ln \frac{1}{R}+k \ln 2}+\frac{\Theta^{\frac{k}{2}}}{\ln \frac{1}{R}}\right]
\end{aligned}
$$

Теперь мы можем оценить сумму в (6.10) следуюшим образом:

$$
\begin{aligned}
\sum_{i=0}^{k-1} \frac{\Theta^{k-1-i}}{\ln ^{2}\left(\frac{2^{i}}{R}\right)} & =\frac{1}{\Theta}\left[\frac{1}{\ln ^{2} \frac{1}{R}}-\frac{1}{\left(\ln \frac{1}{R}+k \ln 2\right)^{2}}\right]+\frac{1}{\Theta} I(k, \Theta, R) \\
& \leqslant \frac{1}{\Theta}\left[\frac{\Theta^{k}}{\ln ^{2} \frac{1}{R}}+\frac{1}{\Theta \ln 2}\left(\frac{1}{\ln \left(\frac{2^{k}}{R}\right)}+\frac{\Theta^{\frac{k}{2}}}{\ln \frac{1}{R}}\right)\right]
\end{aligned}
$$

Отсюда и из (6.10) выводим

$$
\begin{aligned}
G\left(\frac{R}{2^{k}}\right) & \leqslant \Theta^{\frac{k}{2}}\left[\Theta^{\frac{k}{2}} G(R)+\Phi_{7}^{\prime \prime}\left(\frac{\Theta^{\frac{k}{2}}}{\Theta^{2} \frac{1}{R}}+\frac{1}{\Theta^{2} \ln 2 \ln \frac{1}{R}}\right)\right]+\Phi_{7}^{\prime \prime} \frac{1}{\Theta^{2} \ln 2} \cdot \frac{1}{\ln \left(\frac{2^{k}}{R}\right)} \\
& \leqslant \Theta^{\frac{k}{2}}\left[G(R)+\frac{\Phi_{7}^{\prime \prime}}{\Theta \ln \frac{1}{R}}\left(\frac{1}{\ln \frac{1}{R}}+\frac{1}{\Theta \ln 2}\right)\right]+\frac{\Phi_{7}^{\prime \prime}}{\Theta^{2} \ln 2} \frac{1}{\ln \left(\frac{2^{k}}{R}\right)} .
\end{aligned}
$$

Пусть $0<\rho \leqslant R \leqslant 1 / 4$. Выберем число $k$ таким, чтобы

$$
\frac{1}{2^{k+1}}<\frac{\rho}{R} \leqslant \frac{1}{2^{k}}
$$


Тогда

$$
\begin{gathered}
\rho \leqslant \frac{R}{2^{k}}, \quad \frac{1}{2 \rho} \leqslant \frac{2^{k}}{R}, \quad k \leqslant \frac{\ln \frac{R}{\rho}}{\ln 2}, \\
\frac{\ln \frac{R}{2 \rho}}{\ln 2}=\frac{\ln \frac{R}{\rho}}{\ln 2}-1 \leqslant k, \\
\Theta^{\frac{k}{2}} \leqslant \exp \left\{-\frac{1}{2} \ln \frac{1}{\Theta} \cdot \frac{\ln \frac{R}{2 \rho}}{\ln 2}\right\}=\left(\frac{2 \rho}{R}\right)^{\frac{1}{2} \frac{\ln \frac{1}{\Theta}}{\ln 2}}
\end{gathered}
$$

и, следовательно,

$$
\begin{gathered}
G(\rho) \leqslant\left(\frac{2 \rho}{R}\right)^{\frac{1}{2} \frac{\ln \frac{1}{\Theta}}{\ln 2}}\left[G(R)+\frac{\Phi_{7}^{\prime \prime}}{\Theta \ln \frac{1}{R}}\left(\frac{1}{\ln \frac{1}{R}}+\frac{1}{\Theta \ln 2}\right)\right]+\frac{\Phi_{7}^{\prime \prime}}{\Theta^{2} \ln 2} \frac{1}{\ln \frac{1}{2 \rho}}, \\
0<\rho \leqslant R \leqslant \frac{1}{4} .
\end{gathered}
$$

Осталось положить $R=1 / 4$, учесть (6.7) и оценку $G(R) \leqslant \Phi_{7}^{\prime}$, вытекающую из (6.2), и получить требуемое неравенство (1.25). Теорема 1.7 доказана.

\section{§ 7. Формальный вывод оценок (1.28), (1.29) \\ и доказательство следствия 1.2}

Мы предполагаем, что утверждения (1.27) уже доказаны и, более того, что

$$
v_{, j}(t), \partial_{t} v_{, i}(t) \in H^{1}, \quad i, j=1,2,
$$

для п.в. $t \in[0, T]$. Этих предположений достаточно, чтобы вывести оценки (1.28), (1.29). Полное доказательство теоремы 1.8 будет дано в $§ 11$.

Начнем с локального варианта оценки (1.28). В силу сделанных выше предположений из (1.4) вытекает тождество

$$
\begin{gathered}
\left(\partial_{t} v_{, i}(t), w\right)+\left(\left(v_{k}(t) v_{, k}(t)\right)_{, i}, w\right)-\left(\operatorname{div} \sigma_{, i}(t), w\right)=\left(g_{, i}(t), w\right), \\
w \in H, \quad i=1,2,
\end{gathered}
$$

для п.в. $t \in[0, T]$. Для произвольных $x_{0} \in \mathbb{R}^{2}$ и $\left.\left.R \in\right] 0,1 / 4\right]$ воспользуемся обозначениями (4.3). Относительно срезающей функции $\varphi$ предположим, что

$$
\begin{gathered}
\varphi \in C_{0}^{2}\left(\mathbb{R}^{2}\right), \quad 0 \leqslant \varphi \leqslant 1, \\
\varphi \equiv 1 \quad \text { в } \omega\left(x_{0}, r\right), \quad \varphi \equiv 0 \text { вне } w\left(x_{0}, q\right), \\
|\nabla \varphi| \leqslant \frac{C}{q-r}, \quad\left|\nabla^{2} \varphi\right| \leqslant \frac{C}{(q-r)^{2}}, \quad \frac{R}{2} \leqslant r<q \leqslant R .
\end{gathered}
$$


Пусть $\widehat{\varphi}$ - периодическое продолжение $\varphi$, определяемое соотношениями $\left(4.4_{2}\right)$. Напоминаем, что функция $v$ считается продолженной на все $\mathbb{R}^{2}$ периодическим образом. Предположения (7.1) позволяют утверждать, что

$$
\widehat{w}(t)=\operatorname{curl}^{*}\left(\widehat{\varphi}^{4} \operatorname{curl} v_{, i}(t)\right)=-\widehat{\varphi}^{4} \Delta v_{, i}(t)+\operatorname{curl}^{*} \widehat{\varphi}^{4} \operatorname{curl} v_{, i}(t) \in H
$$

при п.в. $t \in[0, T]$. Полагая $w=\widehat{w}(t)$ в $(7.2)$, получим

$$
I_{1}+I_{2}=I_{3}+I_{4}
$$

где

$$
\begin{gathered}
I_{1} \equiv\left(\partial_{t} v_{, i}(t), \widehat{w}(t)\right), \quad I_{2} \equiv-\left(\operatorname{div} \sigma_{, i}(t), \widehat{w}(t)\right), \\
I_{3}=-\left(\left(v_{k}(t) v_{, k}(t)\right)_{, i}, \widehat{w}(t)\right), \quad I_{4}=\left(g_{, i}(t), \widehat{w}(t)\right) .
\end{gathered}
$$

Оцениваем слагаемые в (7.4) по очереди:

$$
\begin{gathered}
I_{1}=\frac{1}{2} \partial_{t}\left\|\varphi^{2} \nabla \operatorname{curl} v(t)\right\|_{\omega\left(x_{0}, R\right)}^{2}, \\
I_{2}=\int_{\omega\left(x_{0}, R\right)} \varphi^{4} \operatorname{div} \sigma_{, i}(t) \cdot \Delta v_{, i}(t) d x \\
+\int_{\omega\left(x_{0}, R\right)} \sigma_{, i}(t): \varepsilon\left(\operatorname{curl}^{*} \varphi^{4} \operatorname{curl} v_{, i}(t)\right) d x=I_{2}^{\prime}+I_{2}^{\prime \prime} .
\end{gathered}
$$

Для $I_{2}^{\prime \prime}$ с учетом (7.3) получаем

$$
\left|I_{2}^{\prime \prime}\right| \leqslant \frac{C}{q-r}\left[\left\|\varphi^{2} \nabla^{3} v(t)\right\|_{\omega\left(x_{0}, R\right)}+\frac{1}{q-r}\left\|\nabla^{2} v(t)\right\|_{\omega\left(x_{0}, R\right)}\right]\left\|\nabla^{2} v(t)\right\|_{\omega\left(x_{0}, R\right)} .
$$

Для того чтобы оценить $I_{2}^{\prime}$, воспользуемся тождеством $\left(2.7_{2}\right)$ :

$$
\begin{aligned}
I_{2}^{\prime}= & \left(\sigma_{, i k}(t), \varphi^{4} \varepsilon\left(v_{, i k}(t)\right)\right)_{\omega\left(x_{0}, R\right)} \\
& +\left(\sigma_{, i}(t), \varepsilon\left(v_{, i k}(t)\right) \varphi_{, k}^{4}\right)_{\omega\left(x_{0}, R\right)}-\left(\sigma_{, i} \nabla \varphi^{4}, \Delta v_{, i}(t)\right)_{\omega\left(x_{0}, R\right)} \\
\geqslant & \left(\sigma_{, i k}, \varphi^{4} \varepsilon\left(v_{, i k}(t)\right)\right)_{\omega\left(x_{0}, R\right)}-\frac{C}{q-r}\left\|\varphi^{2} \nabla^{3} v(t)\right\|_{\omega\left(x_{0}, R\right)}\left\|\nabla^{2} v(t)\right\|_{\omega\left(x_{0}, R\right)}
\end{aligned}
$$

Для вторых производных от $\sigma$ мы имеем

$$
\sigma_{, i k}=\left(\frac{\partial^{2} D}{\partial \varepsilon^{2}}(\varepsilon(v)) \varepsilon\left(v_{, i}\right)\right)_{, k}=\frac{\partial^{2} D}{\partial \varepsilon^{2}}(\varepsilon(v)) \varepsilon\left(v_{, i k}\right)+\left(\frac{\partial^{3} D}{\partial \varepsilon^{3}}(\varepsilon(v)) \varepsilon\left(v_{, k}\right)\right) \varepsilon\left(v_{, i}\right) .
$$


Принимая во внимание условие (1.22) и (1.17), выводим из (7.8) оценку

$$
\begin{aligned}
I_{2}^{\prime} \geqslant & 2 \nu\left\|\varphi^{2} \nabla^{2} \varepsilon(v(t))\right\|_{\omega\left(x_{0}, R\right)}^{2}-C\left\|\varphi \nabla^{2} v(t)\right\|_{4, \omega\left(x_{0}, R\right)}^{2}\left\|\varphi^{2} \nabla^{3} v(t)\right\|_{\omega\left(x_{0}, R\right)}^{2} \\
& -\frac{C}{q-r}\left\|\varphi^{2} \nabla^{3} v(t)\right\|_{\omega\left(x_{0}, R\right)}\left\|\nabla^{2} v(t)\right\|_{\omega\left(x_{0}, R\right)}
\end{aligned}
$$

Интегрирование по частям приводит к неравенству

$$
\begin{aligned}
2\left\|\varphi^{2} \nabla^{2} \varepsilon(v(t))\right\|_{\omega\left(x_{0}, R\right)} \geqslant & \left\|\varphi^{2} \nabla^{3} v(t)\right\|_{\omega\left(x_{0}, R\right)}^{2} \\
& -\frac{C}{q-r}\left\|\varphi^{2} \nabla^{3} v(t)\right\|_{\omega\left(x_{0}, R\right)}\left\|\nabla^{2} v(t)\right\|_{\omega\left(x_{0}, R\right)} .
\end{aligned}
$$

Применяя неравенство Коши-Буняковского и объединяя две последние оценки с (7.7), (7.6), устанавливаем

$$
I_{2} \geqslant \frac{\nu}{4}\left\|\varphi^{2} \nabla^{3} v(t)\right\|_{\omega\left(x_{0}, r\right)}^{2}-\frac{C}{(q-r)^{2}}\left\|\nabla^{2} v(t)\right\|_{\omega\left(x_{0}, R\right)}^{2}-C\left\|\varphi \nabla^{2} v(t)\right\|_{4, \omega\left(x_{0}, R\right)}^{4} .
$$

Осталось воспользоваться неравенством типа (2.6):

$$
\begin{aligned}
& \left\|\varphi \nabla^{2} v(t)\right\|_{4, \omega\left(x_{0}, R\right)}^{4} \leqslant C_{1}\left\|\varphi \nabla^{2} v(t)\right\|_{\omega\left(x_{0}, R\right)}^{2}\left\|\nabla\left(\varphi \nabla^{2} v(t)\right)\right\|_{\omega\left(x_{0}, R\right)}^{2} \\
& \quad \leqslant C_{2}\left\|\nabla^{2} v(t)\right\|_{\omega\left(x_{0}, R\right)}^{2}\left(\left\|\nabla^{3} v(t)\right\|_{\omega\left(x_{0}, q\right)}^{2}+\frac{1}{(q-r)^{2}}\left\|\nabla^{2} v(t)\right\|_{\omega\left(x_{0}, R\right)}^{2}\right),
\end{aligned}
$$

и получить окончательную оценку для $I_{2}$ :

$$
\begin{aligned}
I_{2} \geqslant & \frac{\nu}{4}\left\|\varphi^{2} \nabla^{3} v(t)\right\|_{\omega\left(x_{0}, R\right)}^{2}-\frac{C}{(q-r)^{2}}\left\|\nabla^{2} v(t)\right\|_{\omega\left(x_{0}, R\right)}^{2} \\
& -C\left\|\nabla^{2} v(t)\right\|_{\omega\left(x_{0}, R\right)}^{2}\left[\left\|\nabla^{3} v(t)\right\|_{\omega\left(x_{0}, q\right)}^{2}+\frac{1}{(q-r)^{2}}\left\|\nabla^{2} v(t)\right\|_{\omega\left(x_{0}, R\right)}^{2}\right] .
\end{aligned}
$$

Перейдем к оценке правой части в (7.4):

$$
\begin{gathered}
\left|I_{3}\right|=\mid \int_{\omega\left(x_{0}, R\right)} v_{k}(t) \operatorname{curl} v_{, k}(t)\left(\varphi^{4} \operatorname{curl}\left(v_{, i}(t)\right)_{, i} d x \mid\right. \\
\leqslant C\|v(t)\|_{\infty, \omega}\left[\left\|\varphi^{2} \nabla^{3} v(t)\right\|_{\omega\left(x_{0}, R\right)}\left\|\nabla^{2} v(t)\right\|_{\omega\left(x_{0}, R\right)}\right. \\
\left.\quad+\frac{1}{q-r}\left\|\nabla^{2} v(t)\right\|_{\omega\left(x_{0}, R\right)}^{2}\right]
\end{gathered}
$$

и

$$
\begin{aligned}
\left|I_{4}\right| & =\left|\int_{\omega\left(x_{0}, R\right)} \operatorname{curl} g\left(\varphi^{4} \operatorname{curl} v_{, i}(t)\right)_{, i} d x\right| \\
& \leqslant C\|\operatorname{curl} g\|_{\omega\left(x_{0}, R\right)}\left(\left\|\varphi^{2} \nabla^{3} v(t)\right\|_{\omega\left(x_{0}, R\right)}^{2}+\frac{1}{q-r}\left\|\nabla^{2} v(t)\right\|_{\omega\left(x_{0}, R\right)}^{2}\right)^{\frac{1}{2}}
\end{aligned}
$$


Собирая оценки (7.5), (7.9)-(7.11) и применяя неравенство Коши-Буняковского, выводим из (7.4)

$$
\begin{aligned}
& \partial_{t}\left\|\varphi^{2} \nabla \operatorname{curl} v(t)\right\|_{\omega\left(x_{0}, R\right)}^{2}+\frac{\nu}{4}\left\|\nabla^{3} v(t)\right\|_{\omega\left(x_{0}, r\right)}^{2} \\
& \quad \leqslant C\left\{\left\|\nabla^{2} v(t)\right\|_{\omega\left(x_{0}, R\right)}^{2}\left[\left\|\nabla^{3} v(t)\right\|_{\omega\left(x_{0}, q\right)}^{2}+\frac{1}{(q-r)^{2}}\left\|\nabla^{2} v(t)\right\|_{\omega\left(x_{0}, R\right)}^{2}\right]\right. \\
& \left.\quad+\|\operatorname{curl} g(t)\|_{\omega\left(x_{0}, R\right)}^{2}+\left(\|v(t)\|_{\infty, \omega\left(x_{0}, R\right)}^{2}+\frac{1}{(q-r)^{2}}\right)\left\|\nabla^{2} v(t)\right\|_{\omega\left(x_{0}, R\right)}^{2}\right\} .
\end{aligned}
$$

В силу оценки (1.25) теоремы 1.7 для любого $\delta>0$ мы имеем

$$
\sup _{\delta \leqslant t \leqslant T}\left\|\nabla^{2} v(t)\right\|_{\omega\left(x_{0}, R\right)}^{2} \leqslant \frac{1}{\delta^{5}} \sup _{\delta \leqslant t \leqslant T} t^{5}\left\|\nabla^{2} v(t)\right\|_{\omega\left(x_{0}, R\right)}^{2} \leqslant \frac{1}{\delta^{5}} \Phi_{7} \frac{1}{\ln \frac{1}{R}}, \quad 0<R \leqslant \frac{1}{4} .
$$

Таким образом, сушествует мажоранта

$$
\begin{gathered}
\Phi_{8}^{\prime}=\Phi_{8}^{\prime}\left(T,\|\varphi\|,\|g\|_{L_{2}\left(I ; H^{-1}\right)},\left\|t \partial_{t} g\right\|_{L_{2}\left(I ; H^{-1}\right)},\left\|t^{\frac{1}{2}} g\right\|_{Q_{T}},\right. \\
\left.\left\|t^{\frac{5}{2}} \partial_{t} g\right\|_{Q_{T}},\left\|t^{\frac{5}{2}} g\right\|_{L_{\infty}\left(I ; L_{2+\gamma_{0}}\left(\Omega ; \mathbb{R}^{2}\right)\right)}, \frac{1}{\delta}\right) \geqslant 4
\end{gathered}
$$

такая, что

$$
\left.\left.C \frac{1}{\delta^{5}} \Phi_{7} \frac{1}{\ln \frac{1}{R}} \leqslant \frac{\nu}{8} \quad \forall R \in\right] 0, \frac{1}{\Phi_{8}^{\prime}}\right]
$$

с постоянной $C$ из неравенства (7.12). Итак, из (7.12)-(7.14) следует оценка

$$
\begin{aligned}
\partial_{t}\left\|\varphi^{2} \nabla \operatorname{curl} v(t)\right\|_{\omega\left(x_{0}, R\right)}^{2}+\frac{\nu}{4}\left\|\nabla^{3} v(t)\right\|_{\omega\left(x_{0}, r\right)}^{2} & \\
\leqslant & \frac{\nu}{8}\left\|\nabla^{3} v(t)\right\|_{\omega\left(x_{0}, q\right)}^{2}+C\left[\|\operatorname{curl} g(t)\|_{\omega\left(x_{0}, R\right)}^{2}\right. \\
& \left.+\left(\frac{1}{(q-r)^{2}}+\|v(t)\|_{\infty, \omega\left(x_{0}, R\right)}\right)\left\|\nabla^{2} v(t)\right\|_{\omega\left(x_{0}, R\right)}^{2}\right]
\end{aligned}
$$

для всех $R / 2 \leqslant r<q \leqslant R, 0<R \leqslant 1 / \Phi_{8}^{\prime}$.

Пусть $t_{0} \geqslant \delta+R^{2}$, а $\chi$ - срезаюшая функция, удовлетворяюшая условиям

$$
\chi(t)= \begin{cases}0, & t \leqslant t_{0}-q^{2} \\ \frac{t-t_{0}+q^{2}}{q^{2}-r^{2}}, & t_{0}-q^{2}<t<t_{0}-r^{2} \\ 1, & t \geqslant t_{0}-r^{2}\end{cases}
$$

Положим

$$
\left.Q\left(x_{0}, t_{0}, R\right) \equiv \omega\left(x_{0}, R\right) \times\right] t_{0}-R^{2}, t_{0}[.
$$




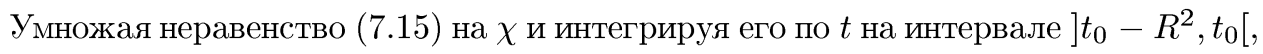
мы получим

$$
\begin{aligned}
G(r) \leqslant & \frac{1}{2} G(q)+C\left[\frac{1}{(q-r)^{2}}+\|v\|_{\infty, Q\left(x_{0}, t_{0}, R\right)}^{2}\right]\left\|\nabla^{2} v\right\|_{Q\left(x_{0}, t_{0}, R\right)}^{2} \\
& +C\|\operatorname{curl} g\|_{Q\left(x_{0}, t_{0}, R\right)}^{2}, \quad \frac{R}{2} \leqslant r<q \leqslant R,
\end{aligned}
$$

где

$$
G(r)=\underset{t_{0}-r^{2} \leqslant t \leqslant t_{0}}{\operatorname{ess} \sup }\|\nabla \operatorname{curl} v(t)\|_{\omega\left(x_{0}, r\right)}^{2}+\frac{\nu}{4}\left\|\nabla^{3} v\right\|_{Q\left(x_{0}, t_{0}, r\right)}^{2} .
$$

Отсюда (см. [17, гл. 5, лемма 3.1]) вытекает

$$
G\left(\frac{R}{2}\right) \leqslant C\left[\frac{1}{R^{2}}+\|v\|_{\infty, Q\left(x_{0}, t_{0}, R\right)}^{2}\right]\left\|\nabla^{2} v\right\|_{Q\left(x_{0}, t_{0}, R\right)}^{2}+C\|\operatorname{curl} g\|_{Q\left(x_{0}, t_{0}, R\right)}^{2}
$$

для всех $x_{0} \in \mathbb{R}^{2}, t_{0} \geqslant R^{2}+\delta, 0<R \leqslant 1 / \Phi_{8}^{\prime}$.

Перейдем к выводу глобальной оценки (1.28). В силу периодичности и оценок (1.12), (5.13) мы имеем

$$
\begin{aligned}
\left\|\nabla^{2} v\right\|_{Q\left(x_{0}, t_{0}, R\right)}^{2} & =\int_{t_{0}-R^{2}}^{t_{0}} \int_{\omega\left(x_{0}, R\right)}\left|\nabla^{2} v(x, t)\right|^{2} d x d t \\
& \leqslant \int_{t_{0}-R^{2}}^{t_{0}} \int_{\Omega}\left|\nabla^{2} v(x, t)\right|^{2} d x d t \\
& \leqslant \int_{\delta}^{T} d t\left\|\nabla^{2} v(t)\right\|^{2} \leqslant \frac{1}{\delta}\left\|\sqrt{t} \nabla^{2} v\right\|_{Q_{T}}^{2} \leqslant \frac{1}{\delta} \Phi_{4}
\end{aligned}
$$

и

$$
\begin{aligned}
\|v\|_{\infty, Q\left(x_{0}, t_{0}, R\right)}^{2} & =\sup _{t_{0}-R^{2} \leqslant t \leqslant t_{0}}\|v(t)\|_{\infty, \omega\left(x_{0}, R\right)}^{2} \\
& \leqslant \sup _{t_{0}-R^{2} \leqslant t \leqslant t_{0}}\|v(t)\|_{\infty}^{2} \leqslant \sup _{\delta \leqslant t \leqslant T}\|v(t)\|_{\infty}^{2} \\
& \leqslant \frac{1}{\delta^{2}} \sup _{\delta \leqslant t \leqslant T}\|t v(t)\|_{\infty}^{2} \leqslant \frac{1}{\delta^{2}}\|t v\|_{\infty, Q_{T}} \leqslant \frac{1}{\delta^{2}}\left(\Phi_{2}^{\prime \prime}\right)^{2} .
\end{aligned}
$$

Таким образом, из (7.18) можно вывести оценку

$$
\begin{aligned}
& \underset{t_{0}-\left(\frac{R}{2}\right)^{2} \leqslant t \leqslant t_{0}}{\operatorname{ess} \sup }\|\nabla \operatorname{curl} v(t)\|_{\omega\left(x_{0}, \frac{R}{2}\right)}^{2}+\frac{\nu}{4}\left\|\nabla^{3} v\right\|_{Q\left(x_{0}, t_{0}, \frac{R}{2}\right)}^{2} \\
& \leqslant C\left[\frac{1}{R^{2}}+\frac{1}{\delta^{2}}\left(\Phi_{2}^{\prime \prime}\right)^{2}\right] \frac{1}{\delta} \Phi_{4}+C\|\operatorname{curl} g\|_{Q_{\delta, T}}^{2}
\end{aligned}
$$

для всех $x_{0} \in \mathbb{R}^{2}, t_{0} \geqslant R^{2}+\delta, 0<R \leqslant 1 / \Phi_{8}$. 
Пусть $\delta_{1}>\delta$, положим

$$
R_{1}=\min \left\{\sqrt{\delta_{1}-\delta}, \frac{1}{\Phi_{8}^{\prime}}\right\}
$$

Затем разобьем отрезки $[-1,1]$ и $\bar{I}_{\delta_{1}}=\left[\delta_{1}, T\right]$ на $N_{1}$ и $N_{2}$ одинаковых частей так, что:

$$
\begin{array}{r}
-1=a_{0}<a_{1}<\cdots<a_{N_{1}}=1, \quad a_{i}-a_{i-1}<\frac{R_{1}}{4}, \quad i=1,2, \ldots, N_{1}, \\
\delta_{1}=t_{0}<t_{1}<\cdots<t_{N_{2}}=T, \quad t_{k}-t_{k-1}<\frac{R_{1}^{2}}{16}, \quad k=1,2, \ldots, N_{2} .
\end{array}
$$

Введем обозначения:

$$
\begin{gathered}
x^{i, j}=\left(a_{i}, a_{j}\right) \in \mathbb{R}^{2}, \quad \omega_{i, j}=\omega\left(x^{i, j}, R_{1}\right), \\
\left.I_{k}=\right] t_{k}-\frac{R_{1}^{2}}{4}, t_{k}\left[, \quad Q_{i, j}^{k}=\omega_{i, j} \times I_{k}, \quad i, j=0,1,2, \ldots, N_{1}, \quad k=0,1,2, \ldots, N_{2} .\right.
\end{gathered}
$$

В силу построения мы имеем

$$
t_{0} \geqslant R_{1}^{2}+\delta, \quad \Omega \subset \bigcup_{i, j=0}^{N_{1}} \omega_{i, j}, \quad I_{\delta_{1}} \subset \bigcup_{k=0}^{N_{2}} I_{k}
$$

и, следовательно,

$$
Q_{\delta_{1}, T} \subset \bigcup_{i, j=0}^{N_{1}} \bigcup_{k=0}^{N_{2}} Q_{i, j}^{k} .
$$

Далее, в силу (7.19) мы имеем

$$
\begin{aligned}
& \operatorname{essup}_{\delta_{1} \leqslant t \leqslant T}\|\nabla \operatorname{curl} v(t)\|_{\Omega}^{2}+\frac{\nu}{4}\left\|\nabla^{3} v\right\|_{Q_{\delta_{1}}, T}^{2} \\
& \leqslant \operatorname{esssup}_{\delta_{1} \leqslant t \leqslant T} \sum_{i, j=0}^{N_{1}}\|\nabla \operatorname{curl} v(t)\|_{\omega_{i, j}}^{2}+\frac{\nu}{4} \sum_{i, j=0}^{N_{1}} \sum_{k=0}^{N_{2}}\left\|\nabla^{3} v\right\|_{Q_{i, j}^{k}}^{2} \\
& \leqslant \sum_{i, j=0}^{N}\left(\underset{\delta_{1} \leqslant t \leqslant T}{\operatorname{ess} \sup }\|\nabla \operatorname{curl} v(t)\|_{\omega_{i, j}}^{2}+\frac{\nu}{4} \sum_{k=0}^{N_{2}}\left\|\nabla^{3} v\right\|_{Q_{i, j}^{k}}^{2}\right) \\
& \leqslant \sum_{i, j=0}^{N_{1}} \sum_{k=0}^{N_{2}}\left[\underset{t \in I_{k}}{\operatorname{ess} \sup }\|\nabla \operatorname{curl} v(t)\|_{\omega_{i, j}}^{2}+\frac{\nu}{4}\left\|\nabla^{2} v\right\|_{Q_{i, j}^{k}}^{2}\right] \\
& \leqslant C N_{1}^{2} N_{2}\left[\frac{1}{\delta} \Phi_{4}\left(\frac{1}{R_{1}^{2}}+\frac{1}{\delta^{2}}\left(\Phi_{2}^{\prime \prime}\right)^{2}\right)+\|\operatorname{curl} g\|_{Q_{\delta, T}}^{2}\right]
\end{aligned}
$$


Осталось заметить, что $\|\nabla \operatorname{curl} v(t)\|=\left\|\nabla^{2} v(t)\right\|$ (см. (2.2)) и что $N_{1}^{2} N_{2} \leqslant \Phi_{8}^{\prime \prime}$ с мажорантой $\Phi_{8}^{\prime \prime}$, зависяшей от тех же переменных, что и $\Phi_{8}$. Итак, формальное доказательство (1.28) закончено.

Перейдем к обсуждению оценки (1.29). Пользуясь предположениями (7.1), мы можем записать тождество

$$
\left(\partial_{t^{2}} v(t), w\right)-\left(\operatorname{div} \partial_{t} \sigma(t), w\right)=\left(\partial_{t} g(t), w\right)-\left(\partial_{t}\left(v_{k}(t) v_{, k}(t)\right), w\right)
$$

для всех $w \in H$ и п.в. всех $t \in[0, T]$. Полагаем

$$
w=\widehat{w}(t)=-\Delta \partial_{t} v(t)
$$

в (7.21), а затем будем оценивать каждое слагаемое в (7.21). Имеем

$$
\left(\partial_{t^{2}} v(t), \widehat{w}(t)\right)=\frac{1}{2}\left\|\nabla \partial_{t} v(t)\right\|^{2}
$$

Далее используем тождество (2.3) и получаем

$$
-\left(\operatorname{div} \partial_{t} \sigma(t), \widehat{w}(t)\right)=\left(\partial_{t} \sigma_{, k}, \varepsilon\left(\partial_{t} v_{, k}(t)\right)\right)
$$

где

$\partial_{t} \sigma_{, k}=\partial_{t}\left(\frac{\partial^{2} D}{\partial \varepsilon^{2}}(\varepsilon(v)) \varepsilon\left(v_{, k}\right)\right)=\frac{\partial^{2} D}{\partial \varepsilon^{2}}(\varepsilon(v)) \varepsilon\left(\partial_{t} v_{, k}\right)+\left(\frac{\partial^{3} D}{\partial \varepsilon^{3}}(\varepsilon(v)) \varepsilon\left(\partial_{t} v\right)\right) \varepsilon\left(v_{, k}\right)$,

и, следовательно, в силу условий $\left(1.2_{3}\right),(1.17)$

$$
\begin{aligned}
-\left(\operatorname{div} \partial_{t} \sigma(t), \widehat{w}(t)\right) & \geqslant 2 \nu\left\|\nabla \varepsilon\left(\partial_{t} v(t)\right)\right\|^{2}-C_{1}\left\|\nabla \varepsilon\left(\partial_{t} v(t)\right)\right\|\left\|\left|\nabla \partial_{t} v\right|\left|\nabla^{2} v\right|\right\| \\
& \geqslant \nu\left\|\nabla \varepsilon\left(\partial_{t} v(t)\right)\right\|^{2}-C_{2}\left\|\nabla \partial_{t} v(t)\right\|_{4}^{2}\left\|\nabla^{2} v(t)\right\|_{4}^{2} \\
& \geqslant \frac{\nu}{2}\left\|\nabla^{2} \partial_{t} v(t)\right\|^{2}-C_{3}\left\|\nabla \partial_{t} v(t)\right\|\left\|\nabla^{2} \partial_{t} v(t)\right\|\left\|\nabla^{2} v(t)\right\|_{4}^{2} \\
& \geqslant \frac{\nu}{4}\left\|\nabla^{2} \partial_{t} v(t)\right\|^{2}-C_{4}\left\|\nabla \partial_{t} v(t)\right\|^{2}\left\|\nabla^{2} v(t)\right\|_{4}^{4}
\end{aligned}
$$

Теперь будем оценивать слагаемые в правой части (7.21). Имеем

$$
\left|\left(\partial_{t} g(t), \widehat{w}(t)\right)\right| \leqslant\left\|\partial_{t} g(t)\right\|\left\|\nabla^{2} \partial_{t} v(t)\right\|
$$

Далее,

$$
\begin{aligned}
& \left|\left(\partial_{t}\left(v_{k}(t) v_{, k}(t)\right), \widehat{w}(t)\right)\right|=\left|\left(\partial_{t}\left(v_{k}(t) v_{, k}(t)\right), \operatorname{curl}{ }^{*}\left(\operatorname{curl} \partial_{t} v(t)\right)\right)\right| \\
& \quad=\left|\left(\partial_{t}\left(v_{k}(t) \operatorname{curl} v_{, k}(t)\right), \operatorname{curl} \partial_{t} v(t)\right)\right|=\left|\left(\partial_{t} v_{k}(t) \operatorname{curl} v_{, k}(t), \operatorname{curl} \partial_{t} v(t)\right)\right| \\
& \quad \leqslant C_{1}\left\|\nabla \partial_{t} v(t)\right\|\left\|\partial_{t} v(t)\right\|_{4}\left\|\nabla^{2} v(t)\right\|_{4} \stackrel{(2.6)}{\leqslant} C_{2}\left\|\nabla \partial_{t} v(t)\right\|^{\frac{3}{2}}\left\|\partial_{t} v(t)\right\|^{\frac{1}{2}}\left\|\nabla^{2} v(t)\right\|_{4} \\
& \quad \stackrel{(2.5)}{\leqslant} C_{3}\left\|\nabla \partial_{t} v(t)\right\|^{2}\left(\left\|\nabla^{2} v(t)\right\|_{4}^{4}+1\right) .
\end{aligned}
$$


Собирая оценки (7.23)-(7.26), выводим из (7.21), (7.22)

$$
\begin{gathered}
\partial_{t}\left\|\nabla \partial_{t} v(t)\right\|^{2}+\frac{\nu}{2}\left\|\nabla^{2} \partial_{t} v(t)\right\|^{2} \leqslant C_{1}\left\{\left\|\nabla \partial_{t} v(t)\right\|^{2}\left(\left\|\nabla^{2} v(t)\right\|_{4}^{4}+1\right)+\left\|\partial_{t} g(t)\right\|^{2}\right\} \\
\leqslant C_{2}\left\{\left\|\nabla \partial_{t} v(t)\right\|^{2}\left(\left\|\nabla^{2} v(t)\right\|^{2}\left\|\nabla^{3} v(t)\right\|^{2}+1\right)+\left\|\partial_{t} g(t)\right\|^{2}\right\} .
\end{gathered}
$$

Пусть срезающая функция $\chi$ выбрана так, чтобы

$$
\chi(t)= \begin{cases}0, & t \leqslant \frac{\delta_{1}+\delta}{2}, \\ \frac{2 t-\delta_{1}-\delta}{\delta_{1}-\delta}, & \frac{\delta_{1}+\delta}{2}<t<\delta_{1}, \\ 1, & t \geqslant \delta_{1} .\end{cases}
$$

Тогда умножение (7.27) на функцию $\chi$, удовлетворяющую условию (7.28), дает

$$
\begin{aligned}
& \partial_{t}\left(\chi(t)\left\|\nabla \partial_{t} v(t)\right\|^{2}\right)+\frac{\nu}{2}\left(\chi(t)\left\|\nabla^{2} \partial_{t} v(t)\right\|^{2}\right) \\
& \leqslant C\left(\chi(t)\left\|\nabla \partial_{t} v(t)\right\|^{2}\right)\left[\left\|\nabla^{2} v(t)\right\|_{2}^{2}\left\|\nabla^{3} v(t)\right\|_{2}^{2}+1\right] \\
& \quad+\chi^{\prime}(t)\left\|\nabla \partial_{t} v(t)\right\|^{2}+C \chi(t)\left\|\partial_{t} g(t)\right\|^{2} .
\end{aligned}
$$

Теперь из (7.29) можно вывести оценку

$$
\begin{aligned}
& \underset{0 \leqslant t \leqslant T}{\operatorname{esssup}}\left(\chi(t)\left\|\nabla \partial_{t} v(t)\right\|^{2}\right) \leqslant C \int_{0}^{T}\left[\chi^{\prime}(t)\left\|\nabla \partial_{t} v(t)\right\|^{2}+\chi(t)\left\|\partial_{t} g(t)\right\|^{2}\right] d x \\
& \quad \times \exp \left\{C\left[\underset{\frac{\delta_{1}+\delta}{2} \leqslant t \leqslant T}{\operatorname{ess} \sup }\left\|\nabla^{2} v(t)\right\|^{2} \int_{\frac{\delta_{1}+\delta}{2}}^{T}\left|\nabla^{3} v(t)\right|^{2} d t+T\right]\right\} .
\end{aligned}
$$

В силу оценки (1.7) и условия (1.20) мы имеем

$$
\begin{gathered}
\int_{0}^{T}\left[\chi^{\prime}(t)\left\|\nabla \partial_{t} v(t)\right\|^{2}+\chi(t)\left\|\partial_{t} g(t)\right\|^{2}\right] d x \leqslant \frac{2}{\left(\delta_{1}-\delta\right) \delta^{2}} \int_{0}^{T}\left\|t \nabla \partial_{t} v(t)\right\|^{2} d t \\
\quad+\frac{1}{\delta^{5}} \int_{0}^{T} t^{5}\left\|\partial_{t} g(t)\right\|^{2} d t \leqslant \frac{2}{\delta^{2}\left(\delta_{1}-\delta\right)} \Phi_{2}+\frac{1}{\delta^{5}}\left\|t^{\frac{5}{2}} \partial_{t} g\right\|_{Q_{T}}^{2} .
\end{gathered}
$$

Таким образом, если воспользоваться оценкой (1.28), в которой $\delta_{1}$ заменено на $\frac{\delta_{1}+\delta}{2}$, мы получим из $(7.30)$ и $(7.31)$

$$
\underset{0 \leqslant t \leqslant T}{\operatorname{ess} \sup }\left(\chi(t)\left\|\partial_{t} v(t)\right\|^{2}\right) \leqslant \Phi_{9}^{\prime},
$$

где мажоранта $\Phi_{9}^{\prime}$ зависит от тех же аргументов, что и $\Phi_{8}$. Но тогда из $(7.29),(7.31)$ и (7.32) следует

$$
\begin{aligned}
\frac{\nu}{2}\left\|\nabla^{2} \partial_{t} v(t)\right\|_{Q_{\delta_{1}, T}}^{2} \leqslant & C \Phi_{9}^{\prime}\left[\operatorname{essip}_{\frac{\delta_{1}+\delta}{2} \leqslant t \leqslant T}\left\|\nabla^{2} v(t)\right\|^{2} \int_{\frac{\delta_{1}+\delta}{2}}^{T}\left\|\nabla^{3} v(t)\right\|^{2} d t+T\right] \\
& +C\left[\frac{2}{\delta^{2}\left(\delta_{1}-\delta\right)} \Phi_{2}+\frac{1}{\delta^{5}}\left\|t^{\frac{5}{2}} \partial_{t} g(t)\right\|_{Q_{T}}^{2}\right] .
\end{aligned}
$$


Повторяя рассуждения, использованные для вывода (7.32), заключаем, что две последние оценки влекут (1.29).

ДоКАЗАТЕЛЬСТво СЛЕДСТвИЯ 1.2. Утверждение (1.30) вытекает из конечности нормы $\left\|\nabla^{2} \partial_{t} v\right\|_{Q_{\delta_{1}}, T}$. Далее в силу неравенства (2.6) имеем

$$
\left\|\nabla^{2} v\right\|_{4, Q_{\delta_{1}, T}}^{2} \leqslant C \sup _{\delta_{1} \leqslant t \leqslant T}\left\|\nabla^{2} v(t)\right\|\left\|\nabla^{3} v\right\|_{Q_{\delta_{1}, T}} \leqslant C \Phi_{8}
$$

и

$$
\left\|\nabla \partial_{t} v\right\|_{4, Q_{\delta_{1}, T}}^{2} \leqslant C \underset{\delta_{1} \leqslant t \leqslant T}{\operatorname{ess} \sup }\left\|\nabla \partial_{t} v(t)\right\|\left\|\nabla^{2} \partial_{t} v\right\|_{Q_{\delta_{1}, T}} \leqslant C \Phi_{9} .
$$

Отсюда вытекает (1.31). Утверждение (1.32) получаем из непрерывности вложения $W_{4}^{1}\left(Q_{\delta_{1}, T} ; \mathbb{R}^{4}\right)$ в $C^{\frac{1}{4}}\left(\bar{Q}_{\delta_{1}, T} ; \mathbb{R}^{4}\right)$ (градиент $\nabla v$ непрерывен по Гёльдеру в $\bar{Q}_{\delta_{1}, T}$ с показателем $1 / 4)$.

\section{§8. Второй способ доказательства конечномерности аттрактора $\mathfrak{M}$}

ДОКАЗАТЕЛЬСТВО ЛЕМмы 1.2. Полагая $w=u(t)$ в (1.36), мы получим

$$
\begin{aligned}
& \frac{1}{2} \partial_{t}\|u(t)\|^{2}+\left(\frac{\partial^{2} D}{\partial \varepsilon^{2}}(\varepsilon(v(t))) \varepsilon(u(t)), \varepsilon(u(t))\right) \\
& \quad=\left(v_{k}(t) u, k(t), u(t)\right)+\left(u_{k}(t) v_{, k}(t), u(t)\right) \leqslant\|\nabla v(t)\|\|u(t)\|_{4}^{2} \\
& \quad \stackrel{(2.6)}{\leqslant} C\|\nabla v(t)\|\|\nabla u(t)\|\|u(t)\| .
\end{aligned}
$$

В силу условия $\left(1.2_{3}\right)$ и тождества (2.1) имеем

$$
\left(\frac{\partial^{2} D}{\partial \varepsilon^{2}}(\varepsilon(v(t))) \varepsilon(u(t)), \varepsilon(u(t))\right) \geqslant 2 \nu\|\varepsilon u(t)\|^{2}=\nu\|\nabla u(t)\|^{2}
$$

Отсюда и из (8.1) выводим

$$
\partial_{t}\|u(t)\|^{2}+\nu\|\nabla u(t)\|^{2} \leqslant C\|\nabla v(t)\|^{2}\|u(t)\|^{2} .
$$

Поскольку $v(t) \in \mathfrak{M}, t \in \mathbb{R}^{1}$, то согласно теореме 1.2 мы получаем из (8.2) оценку

$$
\partial_{t}\|u(t)\|^{2}+\nu\|\nabla u(t)\|^{2} \leqslant C M_{1}\|u(t)\|^{2}
$$

и, следовательно,

$$
\|u(1)\|^{2} \leqslant\|u(0)\|^{2} e^{C M_{1}} \Leftrightarrow\left\|V_{1}^{\prime}(\varphi) \psi\right\|^{2} \leqslant\|\psi\|^{2} e^{C M_{1}}
$$


для всех $\psi \in H$ и $\varphi \in \mathfrak{M}$. Отсюда вытекает утверждение (1.37).

Перейдем к доказательству второго утверждения леммы. Положим

$$
\begin{gathered}
v^{1}(t)=V_{t}\left(\varphi^{1}\right), \quad v(t)=V_{t}(\varphi), \quad \tilde{u}(t)=v^{1}(t)-v(t), \\
u(t)=V_{t}^{\prime}(\varphi)\left(\varphi^{1}-\varphi\right), \quad \hat{u}(t)=\tilde{u}(t)-u(t) .
\end{gathered}
$$

Из тождеств (1.4), (1.36) вытекает

$$
\begin{aligned}
& \left(\partial_{t} \hat{u}(t), w\right)+\left(\frac{\partial D}{\partial \varepsilon}\left(\varepsilon\left(v^{1}(t)\right)\right)-\frac{\partial D}{\partial \varepsilon}(\varepsilon(v(t)))-\frac{\partial^{2} D}{\partial \varepsilon^{2}}(\varepsilon(v(t))) \varepsilon(u(t)), \varepsilon(w)\right) \\
& \quad=-\left(v_{k}^{1}(t) v_{, k}^{1}(t)-v_{k}(t) v_{, k}(t)-v_{k}(t) u_{, k}(t)-u_{k}(t) v_{, k}(t), w\right) .
\end{aligned}
$$

Подставим $w=\hat{u}(t)$ в последнее тождество и применим формулу конечных приращений:

$$
\begin{aligned}
\frac{1}{2} \partial_{t} & \|\hat{u}(t)\|^{2}+\left(\int_{0}^{1}\left(\frac{\partial^{2} D}{\partial \varepsilon^{2}}(\varepsilon(v(t))+\theta \varepsilon(\tilde{u}(t)))-\frac{\partial^{2} D}{\partial \varepsilon^{2}}(\varepsilon(v(t)))\right) d \theta \varepsilon(\tilde{u}(t)), \varepsilon(\hat{u}(t))\right) \\
& +\left(\frac{\partial^{2} D}{\partial \varepsilon^{2}}(\varepsilon(v(t))) \varepsilon(\hat{u}(t)), \varepsilon(\hat{u}(t))\right) \\
= & -\left(v_{k}^{1}(t) \tilde{u}_{, k}(t)+\tilde{u}_{k}(t) v_{, k}(t)-v_{k}(t) u_{, k}(t)-u_{k}(t) v_{, k}(t), \hat{u}(t)\right) \\
= & -\left(\hat{u}_{k}(t) v_{, k}(t), \hat{u}_{k}(t)\right)-\left(v_{k}^{1}(t) \hat{u}_{, k}, \hat{u}(t)\right)-\left(\tilde{u}_{k}(t) u_{, k}(t), \hat{u}(t)\right) \\
\leqslant & \|\nabla v(t)\|\|\hat{u}(t)\|_{4}^{2}+\left(\tilde{u}_{k}(t) \tilde{u}, k(t), \hat{u}(t)\right) \\
\leqslant & \|\nabla v(t)\|\|\hat{u}(t)\|_{4}^{2}+\|\nabla \hat{u}(t)\|\|\tilde{u}(t)\|_{4}^{2} .
\end{aligned}
$$

При оценке второго и третьего слагаемых в левой части (8.4) воспользуемся соответственно условиями (1.17) и (1.23). Правая часть будет оцениваться при помощи неравенства (2.6):

$$
\begin{aligned}
& \frac{1}{2} \partial_{t}\|\hat{u}(t)\|^{2}+2 \nu\|\varepsilon(\hat{u}(t))\|^{2} \leqslant C\|\varepsilon(\tilde{u}(t))\|_{4}^{2}\|\varepsilon(\hat{u}(t))\| \\
& \quad+C\|\nabla v(t)\|\|\hat{u}(t)\|\|\nabla \hat{u}(t)\|+C\|\nabla \hat{u}(t)\|\|\tilde{u}(t)\|\|\nabla \tilde{u}(t)\| .
\end{aligned}
$$

Затем воспользуемся тождеством 2.1, неравенством Коши-Буняковского и теоремой 1.2. В результате будем иметь

$$
\partial_{t}\|\hat{u}(t)\|^{2}+\frac{\nu}{2}\|\nabla \hat{u}(t)\|^{2} \leqslant C\|\varepsilon(\tilde{u}(t))\|_{4}^{4}+C M_{1}\|\hat{u}(t)\|^{2}+C\|\nabla \tilde{u}(t)\|^{2}\|\tilde{u}(t)\|^{2} .
$$

Отсюда выводится оценка

$$
\begin{aligned}
\sup _{0 \leqslant t \leqslant 1}\|\hat{u}(t)\|^{2} \leqslant & C e^{C M_{1}}\left[\int_{0}^{1}\|\varepsilon(\tilde{u}(t))\|_{4}^{4} d t\right. \\
& \left.+\sup _{0 \leqslant t \leqslant 1}\|\tilde{u}(t)\|^{2} \int_{0}^{1}\|\nabla \tilde{u}(t)\|^{2} d t\right] .
\end{aligned}
$$


Принимая во внимание утверждение леммы 4.3, имеем

$$
\|\hat{u}(1)\|^{2} \leqslant C e^{C M_{1}}\left[\int_{0}^{1}\|\varepsilon(\tilde{u}(t))\|_{4}^{4} d t+\left\|\varphi^{1}-\varphi\right\|^{4}\right] .
$$

Для оценки первого слагаемого в правой части (8.5) воспользуемся тем фактом, что

$$
\int_{Q_{1}} \varepsilon(\tilde{u}) d x d t=0
$$

и мультипликативным неравенством (см. [15, гл. ІІ $])$

$$
\begin{aligned}
\|\varepsilon(\tilde{u})\|_{4, Q_{1}} & \leqslant C_{1}\left(\|\nabla \varepsilon(\tilde{u})\|_{4, Q_{1}}+\left\|\partial_{t} \varepsilon(\tilde{u})\right\|_{4, Q_{1}}\right)^{\frac{3}{7}}\|\varepsilon(\tilde{u})\|_{Q_{1}}^{\frac{4}{7}} \\
& \leqslant C_{2}\|\nabla \tilde{u}\|_{W_{4}^{1}\left(Q_{1} ; \mathbb{R}^{4}\right)}^{\frac{3}{7}}\|\nabla \tilde{u}\|_{Q_{1}}^{\frac{4}{7}} .
\end{aligned}
$$

Таким образом, отсюда, а также из (8.5) и леммы 4.3 выводим

$$
\begin{aligned}
\|\hat{u}(1)\|^{2} & \leqslant C e^{C M_{1}}\left\{\|\nabla \tilde{u}\|_{W_{4}^{1}\left(Q_{1} ; \mathbb{R}^{4}\right)}^{\frac{12}{7}}\left\|\varphi^{1}-\varphi\right\|^{\frac{16}{7}}+\left\|\varphi^{1}-\varphi\right\|^{4}\right\} \\
& \leqslant C e^{C M_{1}}\left\|\varphi^{1}-\varphi\right\|^{2 \frac{2}{7}}\left\{\|\nabla \tilde{u}\|_{W_{4}^{1}\left(Q_{1} ; \mathbb{R}^{4}\right)}^{\frac{12}{7}}+\left\|\varphi^{1}-\varphi\right\|^{\frac{12}{7}}\right\} .
\end{aligned}
$$

С другой стороны, в силу оценки (1.34) теоремы 1.9 и согласно теореме 1.2 имеем

$$
\begin{aligned}
\|\nabla \tilde{u}\|_{W_{4}^{1}\left(Q_{1} ; \mathbb{R}^{4}\right)}= & \left(\int _ { 0 } ^ { 1 } \left(\left\|\nabla^{2}\left(V_{t}\left(\varphi^{1}\right)-V_{t}(\varphi)\right)\right\|_{4}^{4}\right.\right. \\
& \left.\left.+\left\|\nabla \partial_{t}\left(V_{t}\left(\varphi^{1}\right)-V_{t}(\varphi)\right)\right\|_{4}^{4}\right) d t\right)^{\frac{1}{4}} \leqslant 2 M_{2}^{\frac{1}{4}}
\end{aligned}
$$

и

$$
\left\|\varphi^{1}-\varphi\right\| \leqslant 2 R_{0}
$$

Собирая три последних оценки, получим

$$
\|\hat{u}(1)\|^{2} \leqslant C e^{C M_{1}}\left\|\varphi^{1}-\varphi\right\|^{2 \frac{2}{7}}\left\{M_{2}^{\frac{3}{7}}+R_{0}^{\frac{12}{7}}\right\}
$$

для всех $\varphi^{1}, \varphi \in \mathfrak{M}$. Осталось положить

$$
r \equiv 1, \quad h(s)=s^{\frac{1}{7}} C e^{C M_{1}}\left\{M_{2}^{\frac{3}{7}}+R_{0}^{\frac{12}{7}}\right\}^{\frac{1}{2}}, \quad s \in[0,1],
$$

вспомнить определение $\hat{u}(1)=V_{1}\left(\varphi^{1}\right)-V_{1}(\varphi)-V_{1}^{\prime}(\varphi)\left(\varphi^{1}-\varphi\right)$ и вывести из (8.6) требуемое неравенство (1.38). Лемма 1.2 доказана. 
ДОКАЗАТЕЛЬСТВО ТЕОРЕМЫ 1.10. Как Уже объяснялось в $§ 1$, мы можем применить схему оценки размерности аттракторов, изложенную в [6]. В частности, мы будем использовать теорему 4.9 из $[6]$ при $t=1 / \nu$. Еше раз подчеркнем, что в силу полугрупповых свойств оператор $V_{t}$ обладает свойством равномерной квазидифференцируемости на $\mathfrak{M}$ при всяком конечном $t$. Для того чтобы использовать теорему 4.9 из [6], мы должны определить мажоранту для

$$
\begin{aligned}
\left(L^{c}(t) w, w\right) & \equiv-((\nabla w) v(t)+(\nabla v(t)) w, w)-\left(\frac{\partial^{2} D}{\partial \varepsilon^{2}}(\varepsilon(v(t))) \varepsilon(w), \varepsilon(w)\right) \\
& \leqslant-((\nabla v(t)) w, w)-2 \nu\|\varepsilon(w)\|^{2} \leqslant\|\nabla v(t)\|\|w\|_{4}^{2}-\nu\|\nabla w\|^{2} \\
& \leqslant \bar{c}\|\nabla v(t)\|\|w\|\|\nabla w\|-\nu\|\nabla w\|^{2} \leqslant-\frac{\nu}{2}\|\nabla w\|^{2}+\frac{\bar{c}^{2}}{2 \nu}\|\nabla v(t)\|^{2}\|w\|^{2},
\end{aligned}
$$

где $\bar{c}$ - абсолютная постоянная из неравенства (2.6). Полагаем

$$
\begin{gathered}
h_{1}(t)=\frac{\nu}{2}, \quad h_{0}(t)=\frac{\bar{c}^{2}}{2 \nu}\|\nabla v(t)\|^{2}, \quad \bar{h}_{1}(t)=\frac{\nu}{2}, \\
\bar{h}_{0}(t)=\frac{\bar{c}^{2}}{2 \nu t} \int_{0}^{t}\|\nabla v(\theta)\|^{2} d \theta=\frac{\bar{c}^{2}}{2} \int_{0}^{t}\|\nabla v(\theta)\|^{2} d \theta, \quad t=\frac{1}{\nu} .
\end{gathered}
$$

С другой стороны, так как $v(t) \in \mathfrak{M}$, то мы можем оценить $\bar{h}_{0}(1 / \nu)$ (см. $[5$, теорема 1.2, оценки 2.4]):

$$
\begin{aligned}
\frac{\nu}{2} \int_{0}^{t}\|\nabla v(\theta)\|^{2} d \theta & \leqslant R_{0}^{2}+\frac{2}{\nu} t\|f\|_{(-1)}^{2}=\left[t=\frac{1}{\nu}\right] \\
& =\frac{4\|f\|_{(-1)}^{2}}{\nu^{2} \lambda_{1}}+\frac{2}{\nu^{2}}\|f\|_{(-1)}^{2}=\frac{2}{\nu^{2}}\|f\|_{(-1)}^{2}\left[\frac{2}{\lambda_{1}}+1\right]
\end{aligned}
$$

и, следовательно,

$$
\bar{h}_{0}(t) \leqslant \frac{2 \bar{c}^{2}}{\nu^{3}}\|f\|_{(-1)}^{2}\left[\frac{2}{\lambda_{1}}+1\right], \quad t=\frac{1}{\nu} .
$$

Известно, что $\lambda_{k}=O(k)$, и потому сушествует абсолютная положительная постоянная $c_{1}$ такая, что

$$
\lambda_{1}+\lambda_{2}+\cdots+\lambda_{n} \geqslant c_{1} n^{2}, \quad n=1,2, \ldots
$$

Здесь $\left\{\lambda_{i}\right\}_{i=1}^{\infty}-$ собственные значения оператора задачи (1.3). Тогда согласно теореме 4.9 из [6] мы имеем оценки

$$
\operatorname{dim}_{H} \mathfrak{M} \leqslant \operatorname{dim}_{f} \mathfrak{M} \leqslant N,
$$

где $N$ удовлетворяет неравенству

$$
-\bar{h}_{1}(t) c_{1} N+\bar{h}_{0}(t)<0, \quad t=\frac{1}{\nu} .
$$

Таким образом,

$$
N<\frac{\bar{h}_{0}(t)}{c_{1} \bar{h}_{1}(t)} \leqslant \frac{4 \bar{c}^{2}}{c_{1}}\left[\frac{2}{\lambda_{1}}+1\right] \frac{1}{\nu^{4}}\|f\|_{(-1)}^{2} .
$$

Теорема 1.10 доказана. 


\section{§9. Доказательство теоремы 1.6}

ДОКАЗАТЕЛЬСТВО ТЕОРЕМЫ 1.6. Идея доказательства теоремы 1.6 изложена в $\S 5$. Но для того чтобы все операции были законными, мы вынуждены заменить одну производную по $t$ конечными разностями. Пусть $t \in] 0, T[, h>0$, причем $t+h \in] 0, T\left[\right.$. Полагая $\Delta_{h} f(t)=(f(t+h)-f(t)) / h$, выводим из вариационного тождества (1.4) следуюшее соотношение:

$$
\begin{gathered}
\left(\Delta_{h} \partial_{t} v(t), w\right)+\left(\Delta_{h} \frac{\partial D}{\partial \varepsilon}(\varepsilon(v(t))), \varepsilon(w)\right)=-\left(\Delta_{h}(\nabla v(t) v(t)), w\right)+\left(\Delta_{h} g(t), w\right) \\
\forall w \in H^{1}
\end{gathered}
$$

Применяя формулу конечных приращений, введем обозначения

$$
\Delta_{h} \frac{\partial^{2} D}{\partial \varepsilon^{2}}(\varepsilon(v(t)))=\mathscr{L}_{h}(t) \varepsilon\left(\Delta_{h} v(t)\right)
$$

где

$$
\mathscr{L}_{h}(t)=\int_{0}^{1} \frac{\partial^{2} D}{\partial \varepsilon^{2}}\left(\varepsilon(v(t))+\theta h \Delta_{h} \varepsilon(v(t))\right) d \theta
$$

причем в силу условий $\left(1.2_{3}\right)$ и $(1.9)$

$$
\begin{gathered}
\left(\mathscr{L}_{h}(t) \varkappa, \varkappa\right) \geqslant 2 \nu|\varkappa|^{2} \quad \forall \varkappa \in \mathbb{M}, \\
\left|\mathscr{L}_{h}(t)\right| \leqslant L .
\end{gathered}
$$

Для произвольного положительного $k$ полагаем

$$
\begin{aligned}
z_{k}(t) & =\min \left\{k,\left|\Delta_{h} v(t)\right|\right\}, \\
w^{k}(t) & =\Delta_{h} v(t) \ln ^{2}\left(2+z_{k}^{2}(t)\right) .
\end{aligned}
$$

В силу теоремы $1.1 w^{k}(t) \in W_{2, \text { per }}^{1}\left(\Omega ; \mathbb{R}^{2}\right)$ для п.в. $t \in[0, T]$ и всех $k$, поскольку

$$
\begin{aligned}
\left|\nabla w^{k}(t)\right| & \leqslant\left|\Delta_{h} \nabla v(t)\right| \ln \left(2+z_{k}^{2}(t)\right)\left[\ln \left(2+z_{k}^{2}(t)\right)+\frac{4\left|\Delta_{h} v(t)\right|^{2}}{2+\left|\Delta_{h} v(t)\right|^{2}}\right] \\
& \leqslant\left|\nabla_{h} \nabla v(t)\right| \ln \left(2+k^{2}\right)\left[\ln \left(2+k^{2}\right)+4\right] .
\end{aligned}
$$

Введем $\bar{w}^{k}(t) \equiv w^{k}(t)-\frac{1}{4} \int_{\Omega} w^{k}(t) d t$; тогда

$$
\left.\bar{w}^{k}(t) \in \stackrel{\circ}{W}=1\left(\Omega ; \mathbb{R}^{2}\right), \quad t \in\right] 0, T[, \quad k>0 .
$$


Теперь вместо задачи (5.1) мы рассматриваем краевую задачу

$$
\left\{\begin{array}{l}
\Delta q=\operatorname{div} w^{k}(t) \text { в } \quad \Omega, \\
\left.q\right|_{x_{i}=-1}=\left.q\right|_{x_{i}=1},\left.\quad \nabla q\right|_{x_{i}=-1}=\left.\nabla q\right|_{x_{i}=1}, \quad i=1,2
\end{array}\right.
$$

решение которой принадлежит $W_{2}^{2}(\Omega)$. Для решения верна оценка

$$
\left\|\nabla^{2} q\right\| \leqslant C_{1}\left\|\operatorname{div} w^{k}(t)\right\| \leqslant C_{2}\left\|\Delta_{h} \nabla v(t) \ln \left(2+z_{k}^{2}(t)\right)\right\| .
$$

Таким образом, мы можем утверждать, что

$$
\widetilde{w}^{k}(t)=\bar{w}^{k}(t)-\nabla q \in H^{1} \quad \text { для п.в. } \quad t \in[0, T] .
$$

Но тогда можно положить $w=\widetilde{w}^{k}(t)$ в (9.1) и получить равенство

$$
I_{1}+I_{2}=I_{3}+I_{4}+I_{5},
$$

в котором

$$
\begin{gathered}
I_{1} \equiv\left(\partial_{t} \Delta_{h} v(t), \widetilde{w}^{k}(t)\right), \quad I_{2} \equiv\left(\mathscr{L}_{h}(t) \varepsilon\left(\Delta_{h} v(t)\right), \varepsilon\left(\widetilde{w}^{k}(t)\right)\right), \\
I_{3} \equiv\left(\left(\nabla \Delta_{h} v(t)\right) v(t+h), \widetilde{w}^{k}(t)\right), \quad I_{4} \equiv\left((\nabla v(t)) \Delta_{h} v(t), \widetilde{w}^{k}(t)\right), \\
I_{5}=\left(\Delta_{h} g(t), \widetilde{w}^{k}(t)\right) .
\end{gathered}
$$

В силу теоремы $1.1 \partial_{t} \Delta_{h} v(T) \in H^{1}$ для п.в. $\left.t \in\right] 0, T[$ таких, что $t+h \in] 0, T[$. По этой причине следующая цепочка равенств законна:

$$
I_{1}=\left(\partial_{t} \Delta_{h} v(t), w^{k}(t)\right)=\frac{1}{2} \partial_{t} \int_{\Omega} H_{k}\left(\left|\partial_{h} v(t)\right|^{2}\right) d x
$$

где (см. (5.6))

$$
H_{k}(y)=\left\{\begin{array}{l}
H_{\infty}(y), \quad 0 \leqslant y \leqslant k^{2}, \\
(y+2) \ln ^{2}\left(2+k^{2}\right)-2\left(2+k^{2}\right)\left[\ln \left(2+k^{2}\right)-1\right], \quad y \geqslant k^{2} .
\end{array}\right.
$$

Для функции $H_{k}$ справедливы соотношения

$$
\begin{gathered}
H_{k}(y) \geqslant H_{\infty}\left(\min \left\{y, k^{2}\right\}\right), \\
H_{k}(y) \rightarrow H_{\infty}(y) \quad \text { при } \quad k \rightarrow+\infty \\
H_{k}(y) \leqslant(y+2) \ln ^{2}(2+y) \leqslant C\left(y^{2}+1\right)
\end{gathered}
$$

для всех $y \geqslant 0$. 
Второе слагаемое в левой части равенства (9.8) оценивается при помощи (9.2), $(9.3),(9.5)-(9.7)$ совершенно так же, как и соответствуюшее слагаемое в (5.5). Выпишем окончательный результат (аналог (5.11)):

$$
I_{2} \geqslant \frac{\nu}{4}\left\|\nabla \Delta_{h} v(t) \ln \left(2+z_{k}^{2}\right)\right\|^{2}-C\left\|\nabla \Delta_{h}(t)\right\|^{2} .
$$

Слагаемые $I_{3}, I_{4}$ оцениваются следующим образом:

$$
\left|I_{3}\right|+\left|I_{4}\right| \leqslant\|v(t+h)\|_{\infty}\left\|\nabla \Delta_{h} v(t)\right\|\left\|\widetilde{w}^{k}(t)\right\|+\left\|\widetilde{w}^{k}(t)\right\|\left\|\Delta_{h} v(t)\right\|_{4}\|\nabla v(t)\|_{4} .
$$

С другой стороны,

$$
\begin{aligned}
\left\|\widetilde{w}^{k}(t)\right\|^{2} & \leqslant\left\|w^{k}(t)\right\|^{2} \leqslant \int_{\Omega}\left|\Delta_{h} v(t)\right|^{2} \ln ^{4}\left(2+z_{k}^{2}(t)\right) \\
& \leqslant \int_{\Omega}\left|\Delta_{h} v(t)\right|^{2} \ln ^{4}\left(2+\left|\Delta_{h} v(t)\right|^{2}\right) d x \\
& \leqslant C\left[\left\|\Delta_{h} v(t)\right\|^{2}+\left\|\Delta_{h} v(t)\right\|_{4}^{4}\right] .
\end{aligned}
$$

Из (9.12) и (9.13) выводим

$$
\begin{aligned}
\left|I_{3}\right|+\left|I_{4}\right| \leqslant & C\left[\left\|\Delta_{h} v(t)\right\|^{2}+\left\|\Delta_{h} v(t)\right\|_{4}^{4}+\|\nabla v(t)\|_{4}^{2}\right. \\
& \left.+\|v(t+h)\|_{\infty}^{2}\left\|\nabla \Delta_{h} v(t)\right\|^{2}\right] .
\end{aligned}
$$

И, наконец, при помощи (9.13) получаем

$$
\left|I_{5}\right| \leqslant C\left\|\Delta_{h} g(t)\right\|\left(\left\|\Delta_{h} v(t)\right\|^{2}+\left\|\Delta_{h} v(t)\right\|_{4}^{4}\right)^{\frac{1}{2}} .
$$

Собирая оценки $(9.9),(9.11),(9.14)$ и (9.15), приходим к неравенству

$$
\begin{gathered}
\partial_{t} \int_{\Omega} H_{k}\left(\left|\Delta_{h} v(t)\right|^{2}\right) d x+\frac{\nu}{2}\left\|\nabla \Delta_{h} v(t) \ln \left(2+z_{k}^{2}(t)\right)\right\|^{2} \\
\leqslant C\left[\left\|\Delta_{h} g(t)\right\|^{2}+\left(1+\|v(t+h)\|_{\infty}^{2}\right)\left\|\nabla \Delta_{h} v(t)\right\|^{2}\right. \\
\left.\quad+\left\|\Delta_{h} v(t)\right\|_{4}^{4}+\|\nabla v(t)\|_{4}^{4}\right]
\end{gathered}
$$

для п.в. $t \in[0, T]$ таких, что $t+h \in] 0, T[$. Пусть теперь $\delta$ - произвольное число из интервала $] 0, T\left[\right.$, и пусть $|h| \leqslant \delta$. Умножая $(9.16)$ на $(t-\delta)^{5}$, мы можем получить 
следующую оценку:

$$
\begin{aligned}
& \operatorname{essip}_{\delta \leqslant t \leqslant T-\delta}(t-\delta)^{5} \int_{\Omega} H_{k}\left(\left|\Delta_{h} v(t)\right|\right) d x \\
& \quad+\frac{\nu}{2} \int_{\delta}^{T-\delta}(t-\delta)^{5}\left\|\nabla \Delta_{h} v(t) \ln \left(2+z_{k}^{2}(t)\right)\right\|^{2} d t \\
& \leqslant C\left[\int_{\delta}^{T-\delta}(t-\delta)^{4} \int_{\Omega} H_{k}\left(\left|\Delta_{h} v(t)\right|^{2}\right) d x d t+\int_{\delta}^{T-\delta}(t-\delta)^{5}\left\|\Delta_{h} g(t)\right\|^{2} d t\right. \\
& +T\left(T^{2}+\sup _{\delta \leqslant t \leqslant t-\delta}(t-\delta)^{2}\|v(t+h)\|_{\infty}^{2}\right) \int_{\delta}^{T-\delta}(t-\delta)^{2}\left\|\nabla \Delta_{h} v(t)\right\|^{2} d t \\
& \left.+T \int_{\delta}^{T-\delta}(t-\delta)^{4}\left\|\Delta_{h} v(t)\right\|_{4}^{4} d t+T^{3} \int_{\delta}^{t-\delta}(t-\delta)^{2}\|\nabla v(t)\|_{4}^{4} d t\right] .
\end{aligned}
$$

В силу (5.12) и (5.13) мы имеем

$$
\sup _{\delta \leqslant t \leqslant(T-\delta)}(t-\delta)\|v(t+h)\|_{\infty} \leqslant \sup _{\delta \leqslant t \leqslant T-\delta}(t+h)\|v(t+h)\|_{\infty} \leqslant\|t v\|_{\infty, Q_{T}} \leqslant \Phi_{2}^{\prime \prime}
$$

И

$$
\int_{\delta}^{T-\delta}(t-\delta)^{2}\|\nabla v(t)\|_{4}^{4} d t \leqslant\|\sqrt{t} \nabla v\|_{4, Q_{T}}^{4} \leqslant\left(\Phi_{2}^{\prime}\right)^{2} .
$$

Принимая во внимание $(9.4),\left(9.10_{1}\right),\left(9.10_{3}\right)$, вьводим из $(9.17)-(9.19)$ следующее соотношение:

$$
\begin{gathered}
\operatorname{ess~sup}_{\delta \leqslant t \leqslant T-\delta}(t-\delta)^{5} \int_{\Omega} H_{\infty}\left(z_{k}^{2}(t)\right)+\frac{\nu}{2} \int_{\delta}^{T-\delta}(t-\delta)^{5}\left\|\nabla \Delta_{h} v(t) \ln \left(2+z_{k}^{2}(t)\right)\right\|^{2} d t \\
\leqslant C\left[T^{4}+(1+T) \int_{\delta}^{T-\delta}(t-\delta)^{4}\left\|\Delta_{h} v(t)\right\|_{4}^{4} d t+\int_{\delta}^{T-\delta}(t-\delta)^{5}\left\|\Delta_{h} g(t)\right\|^{2} d t\right. \\
\left.\quad+T\left(T^{2}+\left(\Phi_{2}^{\prime \prime}\right)^{2}\right) \int_{\delta}^{T-\delta}(t-\delta)^{2}\left\|\nabla \Delta_{h} v(t)\right\|^{2} d t+T^{3}\left(\Phi_{2}^{\prime}\right)^{2}\right]
\end{gathered}
$$

С другой стороны, известно, что

$$
\begin{gathered}
\int_{\delta}^{T-\delta}(t-\delta)^{4}\left\|\Delta_{h} v(t)\right\|_{4}^{4} d t \leqslant\left\|t \partial_{t} v\right\|_{4, Q_{T}}^{4} \stackrel{(5.12)}{\leqslant}\left(\Phi_{2}^{\prime}\right)^{2}, \\
\int_{\delta}^{T-\delta}(t-\delta)^{5}\left\|\Delta_{h} g(t)\right\|^{2} d t \leqslant\left\|t^{\frac{5}{2}} \partial_{t} g\right\|_{Q_{T}}^{2} \\
\int_{\delta}^{T-\delta}(t-\delta)^{2}\left\|\nabla \Delta_{h} v(t)\right\|^{2} d t \leqslant\left\|t \nabla \partial_{t} v\right\|_{Q_{T}}^{2} \stackrel{(1.7)}{\leqslant} \Phi_{2} .
\end{gathered}
$$

Таким образом, три последних неравенства вместе с оценкой (9.20) дают

$$
\begin{aligned}
& \underset{\delta \leqslant t \leqslant T-\delta}{\operatorname{ess} \sup _{\delta}}(t-\delta)^{5} \int_{\Omega} H_{\infty}\left(z_{k}^{2}(t)\right) d x+\frac{\nu}{2} \int_{\delta}^{T-\delta}(t-\delta)^{5}\left\|\nabla \Delta_{h} v(t) \ln \left(2+z_{k}^{2}(t)\right)\right\|^{2} d t \\
& \quad \leqslant C\left[T^{4}+(1+T)\left(\Phi_{2}^{\prime}\right)^{2}+\left\|t^{\frac{5}{2}} \partial_{t} g\right\|_{Q_{T}}^{2}+T^{3}\left(\Phi_{2}^{\prime}\right)^{2}+T\left(T^{2}+\left(\Phi_{2}^{\prime \prime}\right)^{2}\right) \Phi_{2}\right]
\end{aligned}
$$

для всех положительных $k$ и $\delta$. Устремляя $k \mathrm{k}+\infty$, а затем $\delta$ к 0 , заканчиваем доказательство теоремы 1.6. 


\section{§10. Регуляризованная задача}

Используя неравенство

$$
\left\|\nabla^{2} u\right\| \leqslant C\|\Delta u\| \quad \forall u \in H^{2}
$$

введем на $H^{2}$ эквивалентную норму $\|u\|_{(2)}=\|\Delta u\|$.

Для произвольного числа $\mu \in] 0,1]$ рассмотрим следующую задачу. Найти функции $v^{\mu}$ такую, что

$$
\begin{gathered}
v^{\mu} \in C([0, T] ; H) \cap L_{2}\left(0, T ; H^{2}\right), \quad \partial_{t} v^{\mu} \in L_{2}\left(0, T ; H^{-2}\right), \\
\left(\partial_{t} v^{\mu}(t), w\right)+\mu\left(\Delta v^{\mu}(t), \Delta w\right)+\left(\nabla v^{\mu}(t) v^{\mu}(t), w\right)-\left(\operatorname{div} \sigma^{\mu}(t), w\right)=(g(t), w) \\
\forall w \in H^{2}, \quad \sigma^{\mu} \equiv \frac{\partial D}{\partial \varepsilon}\left(\varepsilon\left(v^{\mu}\right)\right),
\end{gathered}
$$

для п.в. $t \in[0, T]$,

$$
v^{\mu}(0)=\varphi
$$

Совершенно так же, как и теорема 1.1, доказывается

Теорема 10.1. Для любьх $T>0, g \in L_{2}\left(0, T ; H^{-1}\right)$ u $\varphi \in H$ существует единственное решение $v^{\mu}$ задачи $\left(10.2_{k}\right), \quad k=1,2,3$. При этом верна оценка

$$
\sup _{0 \leqslant t \leqslant T}\left\|v^{\mu}(t)\right\|^{2}+\mu\left\|\Delta v^{\mu}\right\|_{Q_{T}}^{2}+\left\|\nabla v^{\mu}\right\|_{Q_{T}}^{2}+\left\|\partial_{t} v^{\mu}\right\|_{L_{2}\left(I ; H^{-2}\right)}^{2} \leqslant \Phi_{1}^{\prime} .
$$

Более того, если выполнено условие (1.6), то

$$
\begin{gathered}
\sqrt{t} v^{\mu} \in L_{\infty}\left(I ; H^{1}\right), \quad \sqrt{t} \partial_{t} v^{\mu} \in L_{2}(I ; H), \\
t \partial_{t} v^{\mu} \in L_{\infty}(I ; H) \cap L_{2}\left(I ; H^{1}\right), \\
\sqrt{t} \Delta v^{\mu} \in L_{\infty}(I ; H), \quad t \Delta \partial_{t} v^{\mu} \in L_{2}\left(Q_{T} ; \mathbb{R}^{2}\right)
\end{gathered}
$$

$u$

$$
\begin{aligned}
& \underset{0 \leqslant t \leqslant T}{\operatorname{ess} \sup }\left[t\left\|\nabla v^{\mu}(t)\right\|^{2}+\mu t\left\|\Delta v^{\mu}(t)\right\|^{2}+t^{2}\left\|\partial_{t} v^{\mu}(t)\right\|^{2}\right] \\
& \quad+\int_{0}^{T}\left[t^{2}\left(\mu\left\|\Delta \partial_{t} v^{\mu}(t)\right\|^{2}+\left\|\nabla \partial_{t} v^{\mu}(t)\right\|^{2}\right)+t\left\|\partial_{t} v^{\mu}(t)\right\|^{2}\right] d t \leqslant \Phi_{2}^{\prime}
\end{aligned}
$$

И наконеи, при условии (1.9) $t \partial_{t^{2}} v^{\mu} \in L_{2}\left(I ; H^{-2}\right) u$

$$
\left\|t \partial_{t^{2}} v^{\mu}\right\|_{L_{2}\left(I ; H^{-2}\right)} \leqslant \Phi_{3}^{\prime}
$$

Мажсоранты $\Phi_{1}^{\prime}, \Phi_{2}^{\prime}$ и $\Phi_{3}^{\prime}$ зависят от тех жсе аргументов, что и $\Phi_{1}, \Phi_{2} u \Phi_{3}$ соответственно. 
СЛЕДСТВИЕ 10.1. В условиях теоремы 10.1 мы имеем

$$
\begin{gathered}
t \nabla v^{\mu} \in C\left(\bar{I} ; L_{2}\left(\Omega ; \mathbb{R}^{4}\right)\right), \quad t \Delta v^{\mu} \in C(\bar{I} ; H), \\
t \partial_{t} v^{\mu} \in C(I ; H) .
\end{gathered}
$$

Функции $v^{\mu}$ приближают решение $v$ задачи $\left(1.1_{k}\right), k=1,2, \ldots, 6$, в следующем смысле.

ЛЕмма 10.1. Пусть выполнены все условия теоремы 10.1. Тогда существует подпоследовательность последовательности $v^{\mu}$ (здесь и всюду в дальнейшем все подпоследовательности последовательности $v^{\mu}$ будут снова обозначаться через $\left.v^{\mu}\right)$ такая, что

$$
\begin{array}{rll}
v^{\mu} \rightarrow v & \text { в } & L_{2}\left(I ; H^{1}\right), \\
v^{\mu} \rightarrow v & 8 & L_{2}\left(Q_{T} ; \mathbb{R}^{2}\right), \\
\left(v^{\mu}(t), w\right) \rightarrow(v(t), w) & 8 & C(\bar{I}) \quad \forall w \in H, \\
\partial_{t} v^{\mu} \rightarrow \partial_{t} v & 8 & L_{2}\left(I ; H^{-2}\right), \\
t \partial_{t} v^{\mu} \rightarrow t \partial_{t} v & 8 & L_{2}(I ; H), \\
t \partial_{t} v^{\mu} \rightarrow t \partial_{t} v & 8 & L_{2}\left(I ; H^{1}\right) .
\end{array}
$$

При формулировке леммы 10.1 были использованы символы $\rightarrow$ и $\rightarrow$, обозначаюшие соответственно сильную и слабую сходимости. Лемма 10.1 доказьвается совершенно так же, как и лемма 4.1 в [8].

Используя галёркинские приближения, нетрудно установить аналог теоремы 1.3.

ТЕОРЕМа 10.2. Пусть выполнены все условия теоремы 10.1, а также условие (1.11). Тогда

$$
\left.\left.\sqrt{t} v_{, i j}^{\mu} \in L_{2}\left(I ; H^{2}\right), \quad i, j=1,2, \quad \mu \in\right] 0,1\right],
$$

и имеет место следующая равномерная по $\mu$ оценка:

$$
\left\|\sqrt{t} \nabla^{2} v^{\mu}\right\|_{Q_{T}}+\sqrt{\mu}\left\|\sqrt{t} \nabla \Delta v^{\mu}\right\|_{Q_{T}} \leqslant \Phi_{4}^{\prime}
$$

с мажсорантой $\Phi_{4}^{\prime}$, зависящей от тех же аргументов, что и $\Phi_{4}$.

Теоремы вложения, лемма 10.1, теоремы 10.1 и 10.2 влекут следующие дополнительные утверждения:

$$
\begin{array}{rcc}
\sqrt{t} \nabla^{2} v^{\mu} \rightarrow \sqrt{t} \nabla^{2} v & \text { в } & L_{2}\left(Q_{T} ; \mathbb{R}^{8}\right), \\
t \nabla^{2} v^{\mu} \rightarrow t \nabla^{2} v & \text { в } & L_{2}\left(Q_{T} ; \mathbb{R}^{8}\right), \\
\partial_{t}\left(t \nabla v^{\mu}\right) \rightarrow \partial_{t}(t \nabla v) & \text { в } & L_{2}\left(Q_{T} ; \mathbb{R}^{4}\right), \\
t \nabla v^{\mu} \rightarrow t \nabla v & \text { в } & L_{2}\left(Q_{T} ; \mathbb{R}^{4}\right), \\
\nabla v^{\mu} \rightarrow \nabla v & \text { п.в. в } \quad Q_{T}, \\
\left\|t v^{\mu}\right\|_{W_{4}^{1}\left(Q_{T} ; \mathbb{R}^{2}\right)} \leqslant \Phi_{12}, \\
\left\|t v^{\mu}\right\|_{\infty, Q_{T}} \leqslant \Phi_{13}, \\
t v^{\mu} \rightarrow t v \quad \text { в } \quad C\left(\bar{Q}_{T} ; \mathbb{R}^{2}\right) .
\end{array}
$$


Мажоранты $\Phi_{12}, \Phi_{13}$ зависят от тех же аргументов, что и $\Phi_{4}$.

Следующая лемма является глобальным вариантом леммы 7.1 из [8] для случая периодических краевых условий и доказывается по той же схеме.

ЛЕмма 10.2. Пусть выполнены все условия теоремы 10.2. Тогда

$$
\begin{aligned}
& \rho_{1}(\mu) \equiv \sup _{0 \leqslant t \leqslant T}\left\|t \nabla u^{\mu}(t)\right\|^{2}+\mu\left\|t \nabla \Delta v^{\mu}\right\|_{Q_{T}}^{2}+\left\|t \nabla^{2} u^{\mu}\right\|_{Q_{T}}^{2} \rightarrow 0, \\
& \rho_{2}(\mu) \equiv \sup _{0 \leqslant t \leqslant T}\left\|t^{\frac{3}{2}} \partial_{t} u^{\mu}(t)\right\|^{2}+\mu\left\|t^{\frac{3}{2}} \Delta \partial_{t} v^{\mu}\right\|_{Q_{T}}^{2}+\left\|t^{\frac{3}{2}} \nabla \partial_{t} u^{\mu}\right\|_{Q}^{2} \rightarrow 0
\end{aligned}
$$

прu $\mu \rightarrow 0$. Здесь $u^{\mu}=v^{\mu}-v$.

ДокАЗАТЕЛьСтво. Отметим, что утверждение (10.12) может быть слегка улучшено, а именно:

$$
\sqrt{t} \nabla u^{\mu} \rightarrow 0 \quad \text { в } \quad L_{2}\left(Q ; \mathbb{R}^{4}\right)
$$

Действительно,

$$
\left\|\sqrt{t} \nabla u^{\mu}\right\|_{Q_{T}}^{2} \leqslant\left\|t \nabla u^{\mu}\right\|_{Q_{T}}\left\|\nabla u^{\mu}\right\|_{Q_{T}} \rightarrow 0 \quad \text { при } \quad \mu \rightarrow 0
$$

так как первый сомножитель в правой части последнего неравенства стремится к нулю, а второй ограничен в силу теоремы 10.1 .

Вычтем из тождества $\left(10.2_{2}\right)$ тождество 6.1 , возьмем пробную функцию $w=$ $\Delta v^{\mu}(t)$ и умножим полученное равенство на $t^{2}$. В результате будем иметь

$$
\begin{aligned}
& \mu t^{2}\left\|\nabla \Delta v^{\mu}(t)\right\|^{2}-t^{2}\left(\partial_{t} u^{\mu}(t), \Delta v^{\mu}(t)\right)+t^{2}\left(\operatorname{div}\left(\sigma^{\mu}(t)-\sigma(t)\right), \Delta v^{\mu}(t)\right) \\
& =t^{2}\left(v_{k}^{\mu}(t) v_{, k}^{\mu}(t)-v_{k}(t) v_{, k}^{\mu}(t), \Delta v^{\mu}(t)\right)
\end{aligned}
$$

Перепишем последнее равенство в виде

$$
\begin{aligned}
\frac{1}{2} t^{2} \partial_{t} \| & \nabla u^{\mu}(t)\left\|^{2}+\mu t^{2}\right\| \nabla \Delta v^{\mu}(t) \|^{2}+t^{2}\left(\operatorname{div}\left(\sigma^{\mu}(t)-\sigma(t)\right), \Delta u^{\mu}(t)\right) \\
= & t^{2}\left(\partial_{t} u^{\mu}(t), \Delta v(t)\right)-t^{2}\left(\operatorname{div}\left(\sigma^{\mu}(t)-\sigma(t)\right), \Delta v(t)\right) \\
& \quad+t^{2}\left(v_{k}^{\mu}(t) v_{, k}^{\mu}(t)-v_{k}(t) v_{, k}(t), \Delta v^{\mu}(t)\right) \\
\equiv & t^{2}\left(A_{1}^{\mu}(t)+A_{2}^{\mu}(t)+A_{3}^{\mu}(t)\right)
\end{aligned}
$$


В силу тождества (2.3), условия $\left(1.2_{3}\right)$ получаем

$$
\begin{aligned}
&\left(\operatorname{div}\left(\sigma^{\mu}(t)-\sigma(t)\right), \Delta u^{\mu}(t)\right)=\left(\left(\sigma^{\mu}(t)-\sigma(t)\right)_{, k}, \varepsilon\left(u_{, k}^{\mu}(t)\right)\right) \\
&=\left(\frac{\partial^{2} D}{\partial \varepsilon^{2}}\left(\varepsilon\left(v^{\mu}(t)\right)\right) \varepsilon\left(v_{, k}^{\mu}(t)\right)-\frac{\partial^{2} D}{\partial \varepsilon^{2}}(\varepsilon(v(t))) \varepsilon\left(v_{, k}(t)\right), \varepsilon\left(u_{, k}^{\mu}(t)\right)\right) \\
&=\left(\frac{\partial^{2} D}{\partial \varepsilon^{2}}\left(\varepsilon\left(v^{\mu}(t)\right)\right) \varepsilon\left(u_{, k}^{\mu}(t)\right), \varepsilon\left(u_{, k}^{\mu}(t)\right)\right) \\
& \quad+\left(\left(\frac{\partial^{2} D}{\partial \varepsilon^{2}}\left(\varepsilon\left(v^{\mu}(t)\right)\right)-\frac{\partial^{2} D}{\partial \varepsilon^{2}}(\varepsilon(v(t)))\right) \varepsilon\left(v_{, k}(t)\right), \varepsilon\left(u_{, k}^{\mu}(t)\right)\right) \\
& \geqslant 2 \nu\left\|\nabla \varepsilon\left(u^{\mu}(t)\right)\right\|^{2}-\left\|\nabla \varepsilon\left(u^{\mu}(t)\right)\right\| \\
& \quad \times\left\|\left|\frac{\partial^{2} D}{\partial \varepsilon^{2}}\left(\varepsilon\left(v^{\mu}(t)\right)\right)-\frac{\partial^{2} D}{\partial \varepsilon^{2}}(\varepsilon(v(t)))\right||\nabla \varepsilon(v(t))|\right\| \\
& \geqslant \nu\left\|\nabla \varepsilon\left(u^{\mu}(t)\right)\right\|^{2}-A_{4}^{\mu}(t) \stackrel{(2.1)}{=} \frac{1}{2} \nu\left\|\nabla^{2} u^{\mu}(t)\right\|^{2}-A_{4}^{\mu}(t),
\end{aligned}
$$

где $A_{4}^{\mu} \geqslant 0$ и оценивается следуюшим образом:

$$
\int_{0}^{T} t^{2} A_{4}^{\mu}(t) d t \leqslant C \int_{Q_{T}} t^{2}\left|\frac{\partial^{2} D}{\partial \varepsilon^{2}}\left(\varepsilon\left(v^{\mu}\right)\right)-\frac{\partial^{2} D}{\partial \varepsilon^{2}}(\varepsilon(v))\right|^{2}|\nabla \varepsilon(v)|^{2} d x d t .
$$

Используя (1.12), (10.13), условие (1.9) и теорему Лебега, заключаем, что

$$
\int_{0}^{T} t^{2} A_{4}^{\mu}(t) d t=\int_{0}^{T} t^{2}\left|A_{4}^{\mu}(t)\right| d t \underset{\mu \rightarrow 0}{\rightarrow} 0 .
$$

Теперь из (10.20) и (10.21) выводим оценку

$$
\frac{1}{2} t^{2} \partial_{t}\left\|\nabla u^{\mu}(t)\right\|^{2}+\mu t^{2}\left\|\nabla \Delta v^{\mu}(t)\right\|^{2}+\frac{\nu}{2} t^{2}\left\|\nabla^{2} u^{\mu}(t)\right\|^{2} \leqslant t^{2} \sum_{i=1}^{4} A_{i}^{\mu}(t) .
$$

Далее, согласно (1.12) и (10.75) мы имеем

$$
\int_{0}^{T} t^{2}\left|A_{1}^{\mu}(t)\right| d t \leqslant\left\|t \partial_{t} u^{\mu}\right\|_{Q_{T}}\|t \Delta v\|_{Q_{T} \underset{\mu \rightarrow 0}{\rightarrow} 0 .} 0 .
$$

Оценку $A_{2}^{\mu}$ проведем при помощи тождества (2.3) следующим образом:

$$
\begin{aligned}
\left|\int_{0}^{T} t^{2} A_{2}^{\mu}(t) d t\right|=\left|\int_{0}^{T} t^{2}\left(\sigma_{, k}^{\mu}(t)-\sigma_{, k}(t), \varepsilon\left(v_{, k}(t)\right)\right) d t\right| \\
=\left|\int_{0}^{T} t^{2}\left(\frac{\partial^{2} D}{\partial \varepsilon^{2}} \varepsilon\left(v^{\mu}(t)\right) \varepsilon\left(v_{, k}^{\mu}(t)\right)-\frac{\partial^{2} D}{\partial \varepsilon^{2}}(\varepsilon(v(t))) \varepsilon\left(v_{, k}(t)\right), \varepsilon\left(v_{, k}(t)\right)\right) d t\right| \\
\leqslant\left|\int_{0}^{T} t^{2}\left(\frac{\partial^{2} D}{\partial \varepsilon^{2}}(\varepsilon(v(t))) \varepsilon\left(u_{, k}^{\mu}(t)\right), \varepsilon\left(v_{, k}(t)\right)\right) d t\right| \\
+\mid \int_{0}^{T} t^{2}\left(\left(\frac{\partial^{2} D}{\partial \varepsilon^{2}}\left(\varepsilon\left(v^{\mu}(t)\right)-\frac{\partial^{2} D}{\partial \varepsilon^{2}}(\varepsilon(v(t)))\right) \varepsilon\left(v_{, k}^{\mu}(t)\right), \varepsilon\left(v_{, k}(t)\right)\right) d t \mid\right. \\
\leqslant\left|\int_{0}^{T}\left(\frac{\partial^{2} D}{\partial \varepsilon^{2}}(\varepsilon(v(t))) \varepsilon\left(v_{, k}(t)\right), \varepsilon\left(u_{, k}^{\mu}(t)\right)\right) d t\right| \\
+C\left\|t \nabla^{2} v^{\mu}\right\|_{Q_{T}}\left(\int_{0}^{T} t^{2} A_{4}^{\mu}(t) d t\right)^{\frac{1}{2}} .
\end{aligned}
$$


Первое слагаемое в правой части (10.25) стремится к нулю в силу $\left(10.10_{2}\right)$, а втоpoе - в силу оценки (10.9) и (10.22). Итак,

$$
\int_{0}^{T} t^{2} A_{2}^{\mu}(t) d t \rightarrow 0 \quad \text { при } \quad \mu \rightarrow 0 .
$$

Однако мы не можем пока утверждать, что

$$
\int_{0}^{T} t^{2}\left|A_{2}^{\mu}(t)\right| d t \rightarrow 0 \quad \text { при } \quad \mu \rightarrow 0
$$

Осталось оценить $A_{3}^{\mu}$. Имеем

$$
\begin{aligned}
\int_{0}^{t} t^{2}\left|A_{3}^{\mu}(t)\right| d t \leqslant & \int_{0}^{T} t^{2}\left|\left(\Delta v^{\mu}(t), u_{k}^{\mu}(t) v_{, k}^{\mu}(t)+v_{k}(t) u_{, k}^{\mu}(t)\right)\right| d t \\
\leqslant & \left\|t u^{\mu}\right\|_{\infty, Q_{T}}\left\|\sqrt{t} \nabla^{2} v^{\mu}\right\|_{Q_{T}} \sqrt{T}\left\|\nabla v^{\mu}\right\|_{Q_{T}} \\
& +\|t v\|_{\infty, Q_{T}}\left\|\sqrt{t} \nabla^{2} v^{\mu}\right\|_{Q_{T}}\left\|\sqrt{t} \nabla u^{\mu}\right\|_{Q_{T}} \rightarrow 0
\end{aligned}
$$

при $\mu \rightarrow 0$ в силу $(10.16),(10.9),(10.3)$ и (5.13), (10.9), (10.19) для первого и второго слагаемых в правой части (10.28) соответственно.

Интегрируем неравенство (10.23) по $t$ на $[0, T]$ и получаем

$$
\frac{\nu}{2}\left\|t \nabla^{2} u^{\mu}\right\|_{Q_{T}}^{2} \leqslant \sum_{i=1}^{4} \int_{0}^{T} t^{2} A_{i}^{\mu}(t) d t+\int_{0}^{T} t\left\|\nabla u^{\mu}(t)\right\|^{2} d t \rightarrow 0
$$

при $\mu \rightarrow 0$. Это вытекает из (10.19), (10.22), (10.24), (10.26) и (10.28). Следовательно, $t \nabla^{2} v^{\mu} \rightarrow t \nabla^{2} v$ в $L_{2}\left(Q_{T} ; \mathbb{R}^{2}\right)$. Но тогда из $(10.25)$ вытекает $(10.27)$. Снова интегрируем неравенство (10.23) и приходим к оценке

$$
\begin{aligned}
& \sup _{0 \leqslant t \leqslant T}\left\|t \nabla u^{\mu}(t)\right\|^{2}+\mu\left\|t \nabla \Delta v^{\mu}\right\|_{Q_{T}}^{2}+\nu\left\|t \nabla^{2} u^{\mu}\right\|_{Q_{T}} \\
& \quad \leqslant \sum_{i=1} \int_{0}^{T} t^{2}\left|A_{i}^{\mu}(t)\right| d t+\int_{0}^{T} t\left\|\nabla u^{\mu}(t)\right\|^{2} d t \rightarrow 0 \quad \text { при } \quad \mu \rightarrow 0 .
\end{aligned}
$$

Итак, утверждение (10.17) доказано.

Доказательство (10.18) начнем со следующего замечания. Мы знаем, что $\left\{t \partial_{t} v^{\mu}\right\}$ ограничена в $L_{\infty}(I ; H)$ и $\partial_{t}\left(t \partial_{t} v^{\mu}\right) \rightarrow \partial_{t}\left(t \partial_{t} v\right)$ в $L_{2}\left(I ; H^{-2}\right)$, и, следовательно, $\left(t \partial_{t} v^{\mu}(t), w\right) \rightarrow\left(t \partial_{t} v(t), w\right)$ в $C(\bar{I})$ для любого $w \in H$. Но тогда (см. [8], вывод (7.14))

$$
\left(t \partial_{t} v^{\mu}(t), w(t)\right) \rightarrow\left(t \partial_{t} v(t), w(t)\right) \quad \text { в } \quad C(\bar{I})
$$

для всех $w \in C(I ; H)$. 
Комбинируя тождества для $v^{\mu}$ и $v$, получим

$$
\begin{gathered}
\left(\partial_{t^{2}} u^{\mu}(t), w\right)+\mu\left(\Delta \partial_{t} v^{\mu}(t), \Delta w\right)+\left(\partial_{t} \sigma^{\mu}(t)-\partial_{t} \sigma(t), \varepsilon(w)\right) \\
=\left(\partial_{t}\left(v_{k}^{\mu}(t) u^{\mu}(t)+u_{k}^{\mu}(t) v(t)\right), w_{, k}\right) \quad \forall w \in H^{2} .
\end{gathered}
$$

В силу теоремы 10.2 мы можем положить в последнем тождестве $w=\partial_{t} v^{\mu}(t)$ :

$$
\begin{aligned}
& t^{3}\left(\partial_{t^{2}} u^{\mu}(t), \partial_{t} v^{\mu}(t)\right)+\mu t^{3}\left\|\Delta \partial_{t} v^{\mu}(t)\right\|^{2}+t^{3}\left(\partial_{t} \sigma^{\mu}(t)-\partial_{t} \sigma(t), \varepsilon\left(\partial_{t} v^{\mu}(t)\right)\right) \\
& \quad=t^{3} B_{1}^{\mu}(t) \equiv t^{3}\left(\partial_{t}\left(v_{k}^{\mu}(t) u^{\mu}(t)+u_{k}^{\mu}(t) v(t)\right), \partial_{t} v_{, k}^{\mu}(t)\right) .
\end{aligned}
$$

$B_{1}^{\mu}$ будем оценивать при помощи неравенства (2.6):

$$
\begin{aligned}
\int_{0}^{T} t^{3} \mid & B_{1}^{\mu}(t) \mid d t \leqslant 2 \int_{0}^{T} t^{3}\left\|\nabla \partial_{t} v^{\mu}(t)\right\|\left(\left\|\partial_{t} v^{\mu}(t)\right\|_{4}\left\|u^{\mu}(t)\right\|_{4}+\left\|\partial_{t} u^{\mu}(t)\right\|_{4}\|v(t)\|_{4}\right) \\
\leqslant & C_{1} \int_{0}^{T}\left\|t \nabla \partial_{t} v^{\mu}(t)\right\| t^{2}\left(\left\|\partial_{t} v^{\mu}(t)\right\|^{\frac{1}{2}}\left\|\nabla \partial_{t} v^{\mu}(t)\right\|^{\frac{1}{2}}\left\|u^{\mu}(t)\right\|^{\frac{1}{2}}\left\|\nabla u^{\mu}(t)\right\|^{\frac{1}{2}}\right. \\
& \left.+\left\|\partial_{t} u^{\mu}(t)\right\|^{\frac{1}{2}}\left\|\nabla \partial_{t} u^{\mu}(t)\right\|^{\frac{1}{2}}\|v(t)\|^{\frac{1}{2}}\|\nabla v(t)\|^{\frac{1}{2}}\right) d t \leqslant C_{2} \int_{0}^{T}\left\|t \nabla \partial_{t} v^{\mu}(t)\right\| d t \\
& \times\left\{\sqrt{T}\left\|t \partial_{t} v^{\mu}\right\|_{\infty, Q_{T}}^{\frac{1}{2}}\left(\left\|v^{\mu}\right\|_{\infty, Q_{T}}+\|v\|_{\infty, Q_{T}}\right)^{\frac{1}{2}}\left\|t \nabla \partial_{t} v^{\mu}(t)\right\|^{\frac{1}{2}}\left\|t \nabla u^{\mu}(t)\right\|^{\frac{1}{2}}\right. \\
& \left.+T^{\frac{3}{4}}\|v\|_{\infty, Q_{T}}^{\frac{1}{2}}\left\|t^{\frac{1}{2}} \nabla v\right\|_{\infty, Q_{T}}^{\frac{1}{2}}\left\|t \partial_{t} u^{\mu}(t)\right\|^{\frac{1}{2}}\left\|t \nabla \partial_{t} u^{\mu}(t)\right\|^{\frac{1}{2}}\right\} \\
\leqslant & C_{3} \int_{0}^{T}\left[\sqrt{T}\left\|t \nabla \partial_{t} v^{\mu}(t)\right\|^{\frac{3}{2}}\left\|t \nabla u^{\mu}(t)\right\|^{\frac{1}{2}}\left(\Phi_{2}^{\prime}\right)^{\frac{1}{4}}\left(\left(\Phi_{1}^{\prime}\right)^{\frac{1}{2}}+\Phi_{1}^{\frac{1}{2}}\right)^{\frac{1}{2}}\right. \\
& \left.+T^{\frac{3}{4}}\left\|t \nabla \partial_{t} v^{\mu}(t)\right\|\left\|t \nabla u^{\mu}(t)\right\|^{\frac{1}{2}}\left\|t \nabla \partial_{t} u^{\mu}(t)\right\|^{\frac{1}{2}} \Phi_{1}^{\frac{1}{4}} \Phi_{2}^{\frac{1}{4}}\right] d t \\
\leqslant & \Phi_{14} \int_{0}^{T}\left(\left\|t \nabla \partial_{t} v^{\mu}(t)\right\|^{2}+\left\|t \nabla \partial_{t} v(t)\right\|^{2}\right)^{\frac{3}{4}}\left(\left\|t \nabla u^{\mu}(t)\right\|^{2}+\left\|t \partial_{t} u^{\mu}(t)\right\|^{2}\right)^{\frac{1}{4}} d t \\
\leqslant & \Phi_{14}\left(\left\|t \nabla \partial_{t} v^{\mu}\right\|_{Q_{T}}^{2}+\left\|t \nabla \partial_{t} v\right\|_{Q_{T}}^{2}\right)^{\frac{3}{4}}\left(\left\|t \nabla u^{\mu}(t)\right\|_{Q_{T}}^{2}+\left\|t \partial_{t} u^{\mu}(t)\right\|_{Q_{T}}^{2}\right)^{\frac{1}{2}} \\
\leqslant & \Phi_{14}\left(\Phi_{2}^{\prime}+\Phi_{2}\right)^{\frac{3}{4}}\left(\left\|t \nabla u^{\mu}(t)\right\|_{Q_{T}}^{2}+\left\|t \partial_{t} u^{\mu}(t)\right\|_{Q_{T}}^{2}\right)^{\frac{1}{2}} \rightarrow 0 \quad \text { при } \quad \mu \rightarrow 0 .
\end{aligned}
$$

Утверждение (10.31) вытекает из (10.12) и (10.75).

Преобразуем третье слагаемое в левой части (10.30) следующим образом:

$$
\begin{aligned}
\left(\partial_{t} \sigma^{\mu}(t)\right. & \left.-\partial_{t} \sigma(t), \varepsilon\left(\partial_{t} v^{\mu}(t)\right)\right)=\left(\frac{\partial^{2} D}{\partial \varepsilon^{2}}\left(\varepsilon\left(v^{\mu}(t)\right)\right) \varepsilon\left(\partial_{t} v^{\mu}(t)\right)\right. \\
& \left.-\frac{\partial^{2} D}{\partial \varepsilon^{2}}(\varepsilon(v(t))) \varepsilon\left(\partial_{t} v(t)\right), \varepsilon\left(\partial_{t} v^{\mu}(t)\right)\right)=B_{0}^{\mu}(t)-B_{2}^{\mu}(T)-B_{3}^{\mu}(t)
\end{aligned}
$$


где

$$
\begin{aligned}
& B_{0}^{\mu}(t) \equiv\left(\frac{\partial^{2} D}{\partial \varepsilon^{2}}\left(\varepsilon\left(v^{\mu}(t)\right)\right) \varepsilon\left(\partial_{t} u^{\mu}(t)\right), \varepsilon\left(\partial_{t} u^{\mu}(t)\right)\right) \\
& \geqslant 2 \nu\left\|\varepsilon\left(\partial_{t} u^{\mu}(t)\right)\right\|^{2}=\nu\left\|\nabla \partial_{t} u^{\mu}(t)\right\|^{2} \\
& B_{2}^{\mu}(t) \equiv-\left(\left(\frac{\partial^{2} D}{\partial \varepsilon^{2}}\left(\varepsilon\left(v^{\mu}(t)\right)\right)-\frac{\partial^{2} D}{\partial \varepsilon^{2}}(\varepsilon(v(t)))\right)\right. \\
&\left.\times \varepsilon\left(\partial_{t} v(t)\right), \varepsilon\left(\partial_{t} v^{\mu}(t)\right)+\varepsilon\left(\partial_{t} u^{\mu}(t)\right)\right) \\
& B_{3}^{\mu}(t) \equiv-\left(\frac{\partial^{2} D}{\partial \varepsilon^{2}}(\varepsilon(v(t))) \varepsilon\left(\partial_{t} v(t)\right), \varepsilon\left(\partial_{t} u^{\mu}(t)\right)\right) .
\end{aligned}
$$

Для оценки $B_{2}^{\mu}(t)$ мы снова используем $\left(1.2_{3}\right),(10.13)$ и теорему Лебега:

$$
\begin{aligned}
\int_{0}^{T} t^{3} \mid & B_{2}^{\mu}(t) \mid d t \leqslant \int_{0}^{T} t^{3}\left(\left\|\nabla \partial_{t} v^{\mu}(t)\right\|+\left\|\nabla \partial_{t} u^{\mu}(t)\right\|\right) \\
\times & \left\|\left|\frac{\partial^{2} D}{\partial \varepsilon^{2}}\left(\varepsilon\left(v^{\mu}(t)\right)\right)-\frac{\partial^{2} D}{\partial \varepsilon^{2}}(\varepsilon(v(t)))\right|\left|\varepsilon\left(\partial_{t} v(t)\right)\right|\right\| \\
\leqslant & C\left(\left\|t \nabla \partial_{t} v^{\mu}(t)\right\|_{Q_{T}}^{2}+\left\|t \nabla \partial_{t} u^{\mu}(t)\right\|_{Q_{T}}^{2}\right)^{\frac{1}{2}} \\
& \times\left(\int_{0}^{T} t^{4}\left\|\left|\frac{\partial^{2} D}{\partial \varepsilon^{2}}\left(\varepsilon\left(v^{\mu}(t)\right)\right)-\frac{\partial^{2} D}{\partial \varepsilon^{2}}(\varepsilon(v(t)))\right|\left|\varepsilon\left(\partial_{t} v(t)\right)\right|\right\|^{2} d t\right)_{(10.33)}^{\frac{1}{2}} \rightarrow 0
\end{aligned}
$$

при $\mu \rightarrow 0$. И наконец, в силу $\left(10.7_{6}\right)$

$$
\int_{0}^{T} t^{3} B_{3}^{\mu}(t) d t \rightarrow 0 \quad \text { при } \quad \mu \rightarrow 0 .
$$

Но пока мы не можем утверждать, что

$$
\int_{0}^{T} t^{3}\left|B_{3}^{\mu}(t)\right| d t \rightarrow 0 \quad \text { при } \quad \mu \rightarrow 0 .
$$

Далее, интегрируя по частям, мы получаем

$$
\begin{aligned}
& \int_{0}^{t} \theta^{3}\left(\partial_{t^{2}} u^{\mu}(\theta), \partial_{t} v^{\mu}(\theta)\right) d \theta \\
& \quad=\frac{1}{2} t^{3}\left\|\partial_{t} u^{\mu}(t)\right\|^{2}-\int_{0}^{t} \theta^{3} B_{4}^{\mu}(\theta) d \theta-t^{3} B_{5}^{\mu}(t)-3 \int_{0}^{t} \theta^{2} B_{6}^{\mu}(\theta) d \theta
\end{aligned}
$$

где

$$
\begin{gathered}
B_{4}^{\mu}(t) \equiv\left(\partial_{t^{2}} v(t), \partial_{t} u^{\mu}(t)\right), \quad B_{5}^{\mu}(t)=-\left(\partial_{t} v(t), \partial_{t} u^{\mu}(t)\right) \\
B_{6}^{\mu}(t) \equiv \frac{1}{2}\left[\left\|\partial_{t} v^{\mu}(t)\right\|^{2}-\left\|\partial_{t} v(t)\right\|^{2}\right] .
\end{gathered}
$$


В силу (1.10) и (10.76) мы имеем

$$
\int_{0}^{T} t^{3} B_{4}^{\mu}(t) d t \rightarrow 0 \quad \text { при } \quad \mu \rightarrow 0 .
$$

Однако неясно, верно ли, что

$$
\int_{0}^{T} t^{3}\left|B_{4}^{\mu}(t)\right| d t \rightarrow 0 \quad \text { при } \quad \mu \rightarrow 0 .
$$

В силу $(10.75)$

$$
\int_{0}^{T} t^{2}\left|B_{6}^{\mu}(t)\right| d t \rightarrow 0 \quad \text { при } \quad \mu \rightarrow 0 .
$$

Вспоминая замечание 1.1, отметим, что $t \partial_{t} v \in C(\bar{I} ; H)$, но тогда согласно $(10.29)$

$$
\sup _{0 \leqslant t \leqslant T} t^{3}\left|B_{5}^{\mu}(t)\right| \rightarrow 0 \quad \text { при } \quad \mu \rightarrow 0 .
$$

Теперь из (10.30), (10.32) и (10.36) в результате интегрирования получаем

$$
\begin{gathered}
\frac{1}{2} t^{3}\left\|\partial_{t} u^{\mu}(t)\right\|^{2}+\mu \int_{0}^{t} \theta^{3}\left\|\Delta \partial_{t} v^{\mu}(\theta)\right\|^{2} d \theta+\nu \int_{0}^{t} \theta^{3}\left\|\nabla \partial_{t} u^{\mu}(\theta)\right\|^{2} d \theta \\
\leqslant \sum_{i=1}^{4} \int_{0}^{t} \theta^{3} B_{i}^{\mu}(\theta) d \theta+t^{3} B_{5}^{\mu}(t)+3 \int_{0}^{t} \theta^{2} B_{6}^{\mu}(\theta) d \theta
\end{gathered}
$$

Полагая $t=T$ и принимая во внимание утверждения (10.31), (10.33), (10.34), $(10.37),(10.39)$ и (10.40), заключаем, что

$$
\int_{0}^{T} t^{3}\left\|\nabla \partial_{t} u^{\mu}(t)\right\|^{2} d t \rightarrow 0 \quad \text { при } \quad \mu \rightarrow 0 .
$$

Но это означает, что соотношения (10.35) и (10.38) верны. Из (10.41) следует, что

$$
\begin{aligned}
\frac{1}{2} \sup _{0 \leqslant t \leqslant T} t^{3}\left\|\partial_{t} u^{\mu}(t)\right\|^{2}+\mu \int_{0}^{T} t^{3}\left\|\Delta \partial_{t} v^{\mu}(t)\right\|^{2} d t+\nu \int_{0}^{T} t^{3}\left\|\nabla \partial_{t} u^{\mu}(t)\right\|^{2} d t \\
\leqslant \sum_{i=1}^{4} \int_{0}^{T} t^{3}\left|B_{i}^{\mu}(t)\right| d t+\sup _{0 \leqslant t \leqslant T} t^{3}\left|B_{5}^{\mu}(t)\right| \\
\quad+3 \int_{0}^{T} t^{2}\left|B_{6}^{\mu}(t)\right| d t \rightarrow 0 \quad \text { при } \quad \mu \rightarrow 0 .
\end{aligned}
$$

Итак, утверждение (10.18) доказано. Лемма 10.2 доказана.

Нам понадобится некоторый аналог теоремы 1.7 для решения регуляризованной задачи. 
ЛЕмма 10.3. В условиях теоремы 1.7 для всех $x_{0} \in \mathbb{R}^{2} u 0<R \leqslant 1 / 8$ верна оченка

$$
\sup _{0 \leqslant t \leqslant T}\left\|t^{3} \nabla^{2} v^{\mu}(t)\right\|_{\omega\left(x_{0}, R\right)}^{2} \leqslant \Phi_{15} \frac{1}{\ln \frac{1}{R}}+\frac{1}{R^{4}} \rho_{3}(\mu),
$$

в которой мажсоранта $\Phi_{15}$ зависит от тех же аргументов, что $\Phi_{7}$. При этом

$$
\rho_{3}(\mu) \rightarrow 0 \quad n p u \quad \mu \rightarrow 0
$$

и при фиксированных $T, \varphi, g$, удовлетворяющих условиям леммы, и $\rho_{3}$ не зависит от $R$.

ДокАЗАТЕЛЬСТво. Пусть $x_{0} \in \mathbb{R}^{2}$ и $0<R \leqslant 1 / 8$. Воспользуемся обозначениями (4.3). Пусть срезающая функция $\varphi \in C_{0}^{2}\left(\mathbb{R}^{2}\right)$ удовлетворяет условиям

$$
\begin{gathered}
0 \leqslant \varphi \leqslant 1, \quad \operatorname{spt} \varphi \subset \omega\left(x_{0}, R\right), \\
\varphi \equiv 1 \quad \text { в } \quad \omega\left(x_{0}, \frac{R}{2}\right), \\
|\nabla \varphi| \leqslant \frac{C}{R}, \quad\left|\nabla^{2} \varphi\right| \leqslant \frac{C}{R^{2}} .
\end{gathered}
$$

Пусть, далее, $\widehat{\varphi}$ - периодическое продолжение $\varphi$, определяемое формулой $\left(4.4_{1}\right)$. Положим

$$
\bar{v}(x, t)= \begin{cases}v(x, t)-(\nabla v(t))_{x_{0}, 2 R}\left(x-x_{0}-2 z\right), & x \in \omega^{z}\left(x_{0}, R\right), \quad z \in \mathbb{Z}^{2}, \\ 0, & x \in \mathbb{R}^{2} \backslash \Xi\left(x_{0}, R\right),\end{cases}
$$

где

$$
(\nabla v(t))_{x_{0}, R}=\frac{1}{4 R^{2}} \int_{\omega\left(x_{0}, R\right)} \nabla v(t) d x
$$

И наконец, пусть

$$
\bar{v}^{\mu}=u^{\mu}+\bar{v}
$$

Согласно 10.8 мы утверждаем, что

$$
\widehat{w}(t)=\operatorname{curl}^{*}\left(\widehat{\varphi}^{4} \operatorname{curl} \bar{v}^{\mu}(t)\right) \in H^{2} .
$$

Таким образом, мы можем положить $w=\widehat{w}(t)$ в $\left(10.2_{2}\right)$ и получить

$$
\begin{aligned}
\left(\partial_{t} v^{\mu}(t), \widehat{w}(t)\right)_{\omega\left(x_{0}, 2 R\right)}+\mu\left(\Delta v^{\mu}(t), \Delta \widehat{w}(t)\right)_{\omega\left(x_{0}, 2 R\right)} \\
\quad+\left(\nabla v^{\mu}(t) v^{\mu}(t), \widehat{w}(t)\right)_{\omega\left(x_{0}, 2 R\right)} \\
\quad-\left(\operatorname{div} \sigma^{\mu}(t), \widehat{w}(t)\right)_{\omega\left(x_{0}, 2 R\right)}=(g(t), \widehat{w}(t))_{\omega\left(x_{0}, 2 R\right)} .
\end{aligned}
$$


Слагаемые в (10.46) оцениваются при помоши неравенства Пуанкаре:

$$
\|\nabla \bar{v}(t)\|_{\omega\left(x_{0}, 2 R\right)} \leqslant C R\left\|\nabla^{2} v(t)\right\|_{\omega\left(x_{0}, 2 R\right)}
$$

и равенства

$$
\widehat{w}(t)=-\widehat{\varphi}^{4} \Delta v^{\mu}(t)+\operatorname{curl}^{*} \varphi^{4} \operatorname{curl} \bar{v}^{\mu}(t)
$$

по схеме, которая использовалась для доказательства леммы 7.2 из [8].

Имеем для первого слагаемого в левой части (10.46)

$$
\begin{aligned}
& \left|\left(\partial_{t} v^{\mu}(t), \widehat{w}(t)\right)\right| \leqslant C_{1}\left\|\partial_{t} v^{\mu}(t)\right\|_{\omega\left(x_{0}, 2 R\right)}\left(\left\|\varphi^{2} \Delta v^{\mu}(t)\right\|_{\omega\left(x_{0}, 2 R\right)}^{2}\right. \\
& \left.\quad+\frac{1}{R^{2}}\left\|\nabla \bar{v}^{\mu}(t)\right\|_{\omega\left(x_{0}, 2 R\right)}^{2}\right) \leqslant C_{2}\left(\left\|\partial_{t} v(t)\right\|_{\omega\left(x_{0}, 2 R\right)}^{2}+\left\|\partial_{t} u^{\mu}(t)\right\|_{\omega\left(x_{0}, 2 R\right)}^{2}\right)^{\frac{1}{2}} \\
& \quad \times\left(\left\|\varphi^{2} \Delta v^{\mu}(t)\right\|_{\omega\left(x_{0}, 2 R\right)}^{2}+\left\|\nabla^{2} v(t)\right\|_{\omega\left(x_{0}, 2 R\right)}^{2}+\frac{1}{R^{2}}\left\|\nabla u^{\mu}(t)\right\|^{2}\right)^{\frac{1}{2}}
\end{aligned}
$$

Далее, второе слагаемое в левой части (10.46) оценивается так:

$$
\begin{aligned}
& \mu\left(\Delta v^{\mu}(t), \Delta \widehat{w}(t)\right) \geqslant \frac{\mu}{2}\left\|\varphi^{2} \nabla \Delta v^{\mu}(t)\right\|_{\omega\left(x_{0}, 2 R\right)}^{2}-\frac{\mu C_{1}}{R^{2}}\left[\left\|\Delta v^{\mu}(t)\right\|_{\omega\left(x_{0}, 2 R\right)}^{2}+\frac{1}{R^{2}}\left\|\nabla \bar{v}^{\mu}(t)\right\|_{\omega\left(x_{0}, 2 R\right)}^{2}\right] \\
& \stackrel{(10.44)}{\geqslant} \frac{\mu}{2}\left\|\varphi^{2} \nabla \Delta v^{\mu}(t)\right\|_{\omega\left(x_{0}, 2 R\right)}^{2}-\frac{\mu C_{2}}{R^{4}}\left[\left\|\nabla^{2} v^{\mu}(t)\right\|_{\omega\left(x_{0}, 2 R\right)}^{2}\right. \\
&\left.\quad+\left\|\nabla u^{\mu}(t)\right\|_{\omega\left(x_{0}, 2 R\right)}^{2}+\|\nabla \bar{v}(t)\|_{\omega\left(x_{0}, 2 R\right)}^{2}\right] \\
& \geqslant \frac{\mu}{2}\left\|\varphi^{2} \nabla \Delta v^{\mu}(t)\right\|_{\omega\left(x_{0}, 2 R\right)}^{2}-\frac{\mu C_{3}}{R^{4}}\left[\left\|\nabla^{2} v^{\mu}(t)\right\|^{2}+\left\|\nabla v^{\mu}(t)\right\|^{2}+\|\nabla v(t)\|^{2}\right] \\
& \geqslant \frac{\mu}{2}\left\|\varphi^{2} \nabla \Delta v^{\mu}(t)\right\|_{\omega\left(x_{0}, 2 R\right)}^{2}-\frac{\mu C_{4}}{R^{4}}\left[\left\|\Delta v^{\mu}(t)\right\|^{2}+\|\nabla v(t)\|^{2}\right] .
\end{aligned}
$$

Положим

$$
\rho_{3}^{\prime}(\mu) \equiv \mu \sup _{0 \leqslant t \leqslant T} t^{6}\left[\left\|\Delta v^{\mu}(t)\right\|^{2}+\|\nabla v(t)\|^{2}\right] .
$$

Поскольку

$$
\begin{aligned}
\sup _{0 \leqslant t \leqslant T} t^{6}\left\|\Delta v^{\mu}(t)\right\|^{2} & \leqslant \frac{2}{T}\left\|t^{3} \Delta v^{\mu}\right\|_{Q_{T}}^{2}+T\left\|\partial_{t}\left(t^{3} \Delta v^{\mu}\right)\right\|_{Q_{T}}^{2} \\
& \leqslant T^{4} C\left[\left\|t^{\frac{1}{2}} \Delta v^{\mu}\right\|_{Q_{T}}^{2}+\left\|t^{\frac{3}{2}} \Delta \partial_{t} v^{\mu}\right\|_{Q_{T}}^{2}\right]
\end{aligned}
$$

то в силу $(1.7),(10.18)$ и (10.9) мы получаем

$$
\rho_{3}^{\prime}(\mu) \leqslant \mu T^{2} \Phi_{2}+C T \mu \Phi_{4}^{\prime}+T C \rho_{2}(\mu) \underset{\mu \rightarrow 0}{\rightarrow} 0 .
$$


Итак, мы получили, что

$$
\rho_{3}^{\prime}(\mu) \rightarrow 0 \quad \text { при } \quad \mu \rightarrow 0
$$

и при фиксированных $T, \varphi, g$, удовлетворяющих условиям доказываемой леммы.

Далее, для третьего слагаемого в (10.46) имеем следующую оценку:

$$
\begin{aligned}
& \left|t^{6}\left(v_{k}^{\mu}(t) v_{, k}^{\mu}(t), \widehat{w}(t)\right)\right|=\left|t^{6}\left(v_{k}^{\mu}(t) \operatorname{curl} v_{, k}^{\mu}(t), \varphi^{4} \operatorname{curl} \bar{v}^{\mu}(t)\right)_{\omega\left(x_{0}, 2 R\right)}\right| \\
& \quad=2\left|t^{6}\left(\varphi^{3} v_{k}^{\mu}(t) \varphi_{, k},\left|\operatorname{curl} \bar{v}^{\mu}(t)\right|^{2}\right)_{\omega\left(x_{0}, 2 R\right)}\right| \leqslant C_{1} t^{5}\left\|t v^{\mu}(t)\right\|_{\infty} \frac{1}{R}\left\|\nabla \bar{v}^{\mu}\right\|_{\omega\left(x_{0}, 2 R\right)}^{2} \\
& \quad \stackrel{(10.15)}{\leqslant} C_{2} \Phi_{13} t^{5} \frac{1}{R^{2}}\left(\|\nabla \bar{v}(t)\|_{\omega\left(x_{0}, 2 R\right)}^{2}+\left\|\nabla u^{\mu}(t)\right\|^{2}\right) \\
& \quad \leqslant C_{3} \Phi_{13}\left(\left\|t^{\frac{5}{2}} \nabla^{2} v(t)\right\|_{\omega\left(x_{0}, 2 R\right)}^{2}+\frac{T^{3}}{R^{2}}\left\|t \nabla u^{\mu}(t)\right\|^{2}\right) \\
& \quad \leqslant C_{4} \Phi_{13}\left(\frac{1}{\ln \frac{1}{2 R}} \Phi_{7}+\frac{T^{3}}{R^{2}} \rho_{1}(\mu)\right)
\end{aligned}
$$

для п.в. $t \in[0, T]$.

Правая часть в (10.46) оценивается так же, как и первое слагаемое в левой части (см. (10.47)):

$$
\begin{aligned}
|(g(t), \widehat{w}(t))| \leqslant & C\|g(t)\|_{\omega\left(x_{0}, 2 R\right)}\left(\left\|\varphi^{2} \Delta v^{\mu}(t)\right\|_{\omega\left(x_{0}, 2 R\right)}^{2}\right. \\
& \left.+\left\|\nabla^{2} v(t)\right\|_{\omega\left(x_{0}, 2 R\right)}^{2}+\frac{1}{R^{2}}\left\|\nabla u^{\mu}(t)\right\|^{2}\right)^{\frac{1}{2}} .
\end{aligned}
$$

Осталось оценить последнее слагаемое в левой части (10.46). Имеем

$$
\begin{aligned}
\left|\left(\operatorname{div} \sigma^{\mu}(t), \widehat{w}(t)\right)\right|=\int_{\omega\left(x_{0}, 2 R\right)} \varphi^{4} \operatorname{div} \sigma^{\mu}(t) \cdot \Delta v^{\mu}(t) d x \\
\quad-\frac{C}{R}\left\|\varphi^{2} \nabla^{2} v^{\mu}(t)\right\|_{\omega\left(x_{0}, 2 R\right)}\left\|\nabla \bar{v}^{\mu}(t)\right\|_{\omega\left(x_{0}, 2 R\right)} \\
\quad \begin{array}{l}
2^{\left.2.7_{2}\right)} \\
\geqslant
\end{array} \\
\left.\quad \times \| \nabla \bar{\varphi}^{4} \sigma_{, k}^{\mu}(t), \varepsilon\left(v_{, k}^{\mu}(t)\right)\right)_{\omega\left(x_{0}, 2 R\right)}-\frac{C_{1}}{R}\left\|\varphi^{2} \nabla^{2} v^{\mu}(t)\right\|_{\omega\left(x_{0}, 2 R\right)} \geqslant 2 \nu\left\|\varphi^{2} \nabla \varepsilon\left(v^{\mu}(t)\right)\right\|_{\omega\left(x_{0}, 2 R\right)}^{2} \\
\quad-\frac{C_{2}}{R}\left\|\varphi^{2} \nabla^{2} v^{\mu}(t)\right\|_{\omega\left(x_{0}, 2 R\right)}\left\|\nabla \bar{v}^{\mu}(t)\right\|_{\omega\left(x_{0}, 2 R\right)} \\
\geqslant \frac{\nu}{2}\left\|\varphi^{2} \nabla^{2} v^{\mu}(t)\right\|_{\omega\left(x_{0}, 2 R\right)}^{2}-\frac{C_{3}}{R^{2}}\left\|\nabla \bar{v}^{\mu}(t)\right\|_{\omega\left(x_{0}, 2 R\right)}^{2} \\
\geqslant \frac{\nu}{2}\left\|\varphi^{2} \nabla^{2} v^{\mu}(t)\right\|_{\omega\left(x_{0}, 2 R\right)}^{2} \\
\quad-C_{4}\left[\left\|\nabla^{2} v(t)\right\|_{\omega\left(x_{0}, 2 R\right)}^{2}+\frac{1}{R^{2}}\left\|\nabla u^{\mu}(t)\right\|_{\omega\left(x_{0}, 2 R\right)}^{2}\right] .
\end{aligned}
$$


Теперь из (10.46)-(10.48), (10.50)-(10.52) выводим неравенство

$$
\begin{aligned}
& t^{6}\left(\mu\left\|\varphi^{2} \nabla \Delta v^{\mu}(t)\right\|_{\omega\left(x_{0}, 2 R\right)}^{2}+\nu\left\|\varphi^{2} \nabla^{2} v^{\mu}(t)\right\|_{\omega\left(x_{0}, 2 R\right)}^{2}\right) \\
& \leqslant \frac{C}{R^{4}} \rho_{3}^{\prime}(\mu)+C \Phi_{13}\left(\frac{1}{\ln \frac{1}{R}} \Phi_{7}+T^{3} \rho_{1}(\mu)\right) \\
& \quad+C t^{6}\left[\left\|\nabla^{2} v(t)\right\|_{\omega\left(x_{0}, 2 R\right)}^{2}+\frac{1}{R^{2}}\left\|\nabla u^{\mu}(t)\right\|_{\omega\left(x_{0}, 2 R\right)}^{2}\right] \\
& \quad+C t^{6}\left(\|g(t)\|_{\omega\left(x_{0}, 2 R\right)}^{2}+\left\|\partial_{t} u^{\mu}(t)\right\|_{\omega\left(x_{0}, 2 R\right)}^{2}+\left\|\partial_{t} v(t)\right\|_{\omega\left(x_{0}, w R\right)}^{2}\right)^{\frac{1}{2}} \\
& \quad \times\left(\left\|\varphi^{2} \Delta v^{\mu}(t)\right\|_{\omega\left(x_{0}, 2 R\right)}+\left\|\nabla^{2} v(t)\right\|_{\omega\left(x_{0}, 2 R\right)}^{2}+\frac{1}{R^{2}}\left\|u^{\mu}(t)\right\|_{\omega\left(x_{0}, R\right)}^{2}\right) .
\end{aligned}
$$

Применим неравенство Коши; тогда с учетом (1.23), (1.24), (10.17) и (10.18) получим

$$
\begin{array}{rl}
t^{6}\left(\mu\left\|\nabla \Delta v^{\mu}(t)\right\|_{\omega\left(x_{0}, R\right)}^{2}+\nu\left\|\nabla^{2} v^{\mu}(t)\right\|_{\omega\left(x_{0}, R\right)}^{2}\right) \\
\leqslant C & C \frac{1}{R^{4}} \rho_{3}^{\prime}(\mu)+T^{3} \rho_{1}(\mu)\left[\Phi_{13}+T\right] \\
& \left.+\frac{\Phi_{7}}{\ln \frac{1}{R}}\left[\Phi_{13}+T\right]+\frac{\Phi_{6}}{\ln ^{2} \frac{1}{R}} T+T\left\|t^{\frac{5}{2}} \partial_{t} g(t)\right\|_{\omega\left(x_{0}, 2 R\right)}^{2}\right\} .
\end{array}
$$

Используя условие (1.24), отсюда получаем утверждение леммы 10.3.

\section{§11. Доказательство теоремы 1.8}

Мы начнем с доказательства оценки (1.28). Для этого достаточно повторить соответствуюшие рассуждения $\S 7$, заменяя исходную задачу регуляризованной. При этом вместо теоремы 1.7 мы будем использовать лемму 10.3. В силу (10.8) все рассуждения $\S 7$ будут законны.

Итак, пусть $x_{0} \in \mathbb{R}^{2}$ и $0<R \leqslant 1 / 8$ произвольны. Возьмем срезающую функцию, удовлетворяюшую условиям (7.3). Из вариационного тождества $\left(10.2_{2}\right)$ выводим

$$
\begin{aligned}
& \left(\partial_{t} v_{, i}^{\mu}(t), w\right)-\mu\left(\nabla \Delta v_{, i}^{\mu}(t), \nabla w\right)-\left(\operatorname{div} \sigma_{, i}^{\mu}(t), w\right) \\
& \quad=-\left(\left(v_{k}^{\mu}(t) v_{, k}^{\mu}(t)_{, i}, w\right)+\left(g_{, i}(t), w\right) \quad \forall w \in H^{1}\right.
\end{aligned}
$$

для п.в. $t \in[0, T]$. Согласно (10.8) мы можем положить в $(11.1) w=\widehat{w}(t)$, где

$$
\widehat{w}(t)=\operatorname{curl}^{*}\left(\widehat{\varphi}^{4} \operatorname{curl} v_{, i}^{\mu}(t)\right) \in H^{1}
$$

для п.в. $t \in[0, T]$. Полученное равенство запишем в виде

$$
I_{1}+I_{0}+I_{2}=I_{3}+I_{4}
$$


где

$$
\begin{gathered}
I_{0} \equiv-\mu\left(\nabla \Delta v_{, i}^{\mu}(t), \widehat{w}(t)\right), \quad I_{1} \equiv\left(\partial_{t} v_{, i}(t), \widehat{w}(t)\right), \\
I_{2}=-\left(\operatorname{div} \sigma_{, i}^{\mu}(t), \widehat{w}(t)\right), \quad I_{3} \equiv-\left(\left(v_{k}^{\mu}(t) v_{, k}^{\mu}(t)\right)_{, i}, \widehat{w}(t)\right), \\
I_{4}=\left(g_{, i}(t), \widehat{w}(t)\right) .
\end{gathered}
$$

Величины $I_{k}, k=1,2,3,4$, оцениваются совершенно так же, как и соответствуюшие величины в $\S 7$. Выпишем результат:

$$
\begin{gathered}
I_{1}=\frac{1}{2} \partial_{t}\left\|\varphi^{2} \nabla \operatorname{curl} v^{\mu}(t)\right\|_{\omega\left(x_{0}, R\right)}^{2} \\
I_{2} \geqslant \frac{\nu}{4}\left\|\varphi^{2} \nabla^{3} v^{\mu}(t)\right\|_{\omega\left(x_{0}, R\right)}^{2}-\frac{C}{(q-r)^{2}}\left\|\nabla^{2} v^{\mu}(t)\right\|_{\omega\left(x_{0}, R\right)}^{2} \\
-C\left\|\nabla^{2} v^{\mu}(t)\right\|_{\omega\left(x_{0}, R\right)}^{2}\left[\left\|\nabla^{3} v^{\mu}(t)\right\|_{\omega\left(x_{0}, R\right)}^{2}\right. \\
\left.+\frac{1}{(q-r)^{2}}\left\|\nabla^{2} v^{\mu}(t)\right\|_{\omega\left(x_{0}, R\right)}^{2}\right] \\
\left|I_{3}\right| \leqslant \\
C\left\|v^{\mu}(t)\right\|_{\infty, \omega\left(x_{0}, R\right)}\left[\left\|\varphi^{2} \nabla^{3} v^{\mu}(t)\right\|_{\omega\left(x_{0}, R\right)}\left\|\nabla^{2} v^{\mu}(t)\right\|_{\omega\left(x_{0}, R\right)}\right. \\
\left.+\frac{1}{q-r}\left\|\nabla^{2} v^{\mu}(t)\right\|_{\omega\left(x_{0}, R\right)}^{2}\right] \\
\left|I_{4}\right| \leqslant C\|\operatorname{curl} g\|_{\omega\left(x_{0}, R\right)}\left(\left\|\varphi^{2} \nabla^{3} v^{\mu}(t)\right\|_{\omega\left(x_{0}, R\right)}^{2}\right. \\
\left.+\frac{1}{(q-r)^{2}}\left\|\nabla^{2} v^{\mu}(t)\right\|_{\omega\left(x_{0}, R\right)}^{2}\right)
\end{gathered}
$$

Осталось оценить $I_{0}$. Имеем

$$
\begin{aligned}
I_{0}= & -\mu\left(\nabla \Delta v_{, i}^{\mu}(t),-\varphi^{4} \nabla \Delta v_{, i}^{\mu}-\Delta v_{, i}^{\mu} \otimes \nabla \varphi^{4}\right. \\
& \left.+\nabla \operatorname{curl}^{*} \varphi^{4} \operatorname{curl} v_{, i}^{\mu}(t)+\operatorname{curl}^{*} \varphi^{4} \otimes \nabla \operatorname{curl} v_{, i}^{\mu}(t)\right)_{\omega\left(x_{0}, R\right)} \\
\geqslant & \mu\left\|\varphi^{2} \nabla^{2} \Delta v^{\mu}(t)\right\|_{\omega\left(x_{0}, R\right)}^{2}-\mu C_{1}\left\|\varphi^{2} \nabla^{2} \Delta v^{\mu}(t)\right\|_{\omega\left(x_{0}, R\right)} \\
& \times\left(\frac{1}{(q-r)^{2}}\left\|\nabla^{3} v^{\mu}(t)\right\|^{2}+\frac{1}{(q-r)^{4}}\left\|\nabla^{2} v^{\mu}(t)\right\|^{2}\right)^{\frac{1}{2}} \\
\geqslant & \frac{\mu}{2}\left\|\varphi^{2} \nabla^{2} \Delta v^{\mu}(t)\right\|_{\omega\left(x_{0}, R\right)}^{2}-\frac{\mu C_{2}}{(q-r)^{4}}\left\|\nabla \Delta v^{\mu}(t)\right\|^{2}
\end{aligned}
$$


Собирая (11.3)-(11.7), выводим из (11.2)

$$
\begin{aligned}
\partial_{t} \| \varphi^{2} \nabla & \operatorname{curl} v^{\mu}(t)\left\|_{\omega\left(x_{0}, R\right)}^{2}+\mu\right\| \varphi^{2} \nabla^{2} \Delta v^{\mu}(t) \|_{\omega\left(x_{0}, R\right)}^{2} \\
& +\frac{\nu}{4}\left\|\varphi^{2} \nabla^{3} v^{\mu}(t)\right\|_{\omega\left(x_{0}, R\right)}^{2} \leqslant \mu \frac{C}{(q-r)^{4}}\left\|\nabla \Delta v^{\mu}(t)\right\|^{2} \\
& +C\left\|\nabla^{2} v^{\mu}(t)\right\|_{\omega\left(x_{0}, R\right)}^{2}\left[\left\|\nabla^{3} v^{\mu}(t)\right\|_{\omega\left(x_{0}, q\right)}^{2}+\frac{1}{(q-r)^{2}}\left\|\nabla^{2} v^{\mu}(t)\right\|_{\omega\left(x_{0}, R\right)}^{2}\right] \\
& +C\left(\|v(t)\|_{\infty, \omega\left(x_{0}, R\right)}^{2}+\frac{1}{(q-r)^{2}}\right)\left\|\nabla^{2} v^{\mu}(t)\right\|_{\omega\left(x_{0}, R\right)}^{2} \\
& +C\|\operatorname{curl} g\|_{\omega\left(x_{0}, R\right)}^{2} .
\end{aligned}
$$

Применим лемму 10.3. Тогда для любого $\delta>0$ мы имеем оценку

$$
\sup _{\delta \leqslant t \leqslant T}\left\|\nabla^{2} v^{\mu}(t)\right\|_{\omega\left(x_{0}, R\right)}^{2} \leqslant \frac{1}{\delta^{6}}\left(\Phi_{15} \frac{1}{\ln \frac{1}{R}}+\frac{1}{R^{4}} \rho_{3}(\mu)\right)
$$

для всех $R \in] 0,1 / 8\left[\right.$. Таким образом, сушествует мажоранта $\Phi_{16}$, зависящая от тех же аргументов, что и $\Phi_{8}^{\prime}($ см. $\S 7)$, такая, что

$$
\Phi_{16} \geqslant 8, \quad \frac{C}{\delta^{6}} \Phi_{15} \frac{1}{\ln \frac{1}{R}} \leqslant \frac{\nu}{16}
$$

для всех $R \in] 0, \frac{1}{\Phi_{16}}[$.

Пусть теперь число $R$ из интервала $] 0, \frac{1}{\Phi_{16}}[$ произвольно, но фиксировано. В силу $\left(10.42_{2}\right)$ сушествует положительное число $\mu_{0}$, зависящее от $R$ и аргументов функции $\Phi_{15}$, такое, что

$$
\frac{C}{\delta^{6} R^{4}} \rho_{3}(\mu) \leqslant \frac{\nu}{16} \quad \text { для всех } \quad 0<\mu \leqslant \mu_{0} .
$$

Таким образом, объединяя (11.8)-(1.11), устанавливаем оценку

$$
\begin{aligned}
\partial_{t}\left\|\varphi^{2} \nabla \operatorname{curl} v^{\mu}(t)\right\|_{\omega\left(x_{0}, R\right)}^{2}+\frac{\nu}{4}\left\|\nabla^{3} v^{\mu}(t)\right\|_{\omega\left(x_{0}, r\right)}^{2} \\
\leqslant \frac{\nu}{8}\left\|\nabla^{3} v^{\mu}(t)\right\|_{\omega\left(x_{0}, q\right)}+C\left\{\frac{\mu}{(q-r)^{4}}\left\|\nabla \Delta v^{\mu}(t)\right\|^{2}+\|\operatorname{curl} g(t)\|_{\omega\left(x_{0}, R\right)}^{2}\right. \\
\left.\quad+\left(\left\|v^{\mu}(t)\right\|_{\infty}^{2}+\frac{1}{(q-r)^{2}}\right)\left\|\nabla^{2} v^{\mu}(t)\right\|_{\omega\left(x_{0}, R\right)}^{2}\right\}
\end{aligned}
$$

для п.в. $t \geqslant \delta, \frac{R}{2} \leqslant q<r \leqslant R, 0<R \leqslant \frac{1}{\Phi_{16}}, 0<\mu \leqslant \mu_{0}$. 
Пусть $t_{0} \geqslant \delta+R^{2}$. Рассуждая так же, как и в $\S 7$, выводим из (11.12)

$$
\begin{aligned}
G^{\mu}(r) \leqslant & C \frac{1}{R^{4}}\left\|\nabla \Delta v^{\mu}\right\|_{\Omega \times] t_{0}-R^{2}, t_{0}[}^{2}+C\left\{\left[\frac{1}{R^{2}}+\left\|v_{\infty}^{\mu}\right\|_{\infty, Q\left(x_{0}, t_{0}, R\right)}^{2}\right]\right. \\
\times & \left.\left\|\nabla^{2} v^{\mu}\right\|_{Q\left(x_{0}, t_{0}, R\right)}^{2}+\|\operatorname{curl} g\|_{Q\left(x_{0}, t_{0}, R\right)}^{2}\right\} \\
& \left.t_{0} \geqslant \delta+R^{2}, \quad R \in\right] 0, \frac{1}{\Phi_{16}}\left[, \quad 0<\mu \leqslant \mu_{0} .\right.
\end{aligned}
$$

Согласно лемме 10.2 мы имеем

$$
\begin{gathered}
\mu\left\|\nabla \Delta v^{\mu}\right\|_{\Omega \times I_{\delta}}^{2} \leqslant \frac{1}{\delta^{2}} \rho_{1}(\mu) \underset{\mu \rightarrow 0}{\rightarrow} 0 \\
\left\|\nabla^{2} v^{\mu}\right\|_{Q\left(x_{0}, t_{0}, R\right)} \leqslant\left\|\nabla^{2} v\right\|_{Q\left(x_{0}, t_{0}, R\right)}+\left\|\nabla^{2} u^{\mu}\right\|_{\Omega \times I_{\delta}} \\
\leqslant\left\|\nabla^{2} v\right\|_{Q\left(x_{0}, t_{0}, R\right)}+\frac{1}{\delta} \sqrt{\rho_{1}(\mu)} .
\end{gathered}
$$

Переходя к пределу в (11.13) с учетом (11.14), (10.16), получаем оценку (7.18), в которой надо заменить $\Phi_{8}^{\prime}$ на $\Phi_{16}$. Далее просто повторяя рассуждения $\S 7$, получаем (1.28).

Перейдем к доказательству (1.29). Для того чтобы сделать рассуждения $\S 7$ неформальными, достаточно одну производную по времени заменить разностным отношением. Перепишем (9.1) в виде

$$
\left(\partial_{t} \Delta_{h} v(t), w\right)-\left(\operatorname{div} \Delta_{h} \sigma(t), w\right)=-\left(\Delta_{h}(\nabla v(t) v(t)), w\right)+\left(\Delta_{h} g(t), w\right) \quad \forall w \in H,
$$

для п.в. всех $t \in[0, T]$ таких, что $t, t+h \in[0, T]$, и положим

$$
w=\widehat{w}(t)=-\Delta \Delta_{h} v(t) .
$$

Будем оценивать каждое слагаемое отдельно:

$$
\left(\partial_{t} \Delta_{h} v(t), \widehat{w}(t)\right)=\frac{1}{2} \partial_{t}\left\|\nabla \Delta_{h} v(t)\right\|^{2} .
$$

В силу тождества (2.3) имеем

$$
\begin{aligned}
& -\left(\operatorname{div} \Delta_{h} \sigma(t), \widehat{w}(t)\right)=\left(\Delta_{h} \sigma_{, k}(t), \varepsilon\left(\Delta_{h} v_{, k}(t)\right)\right) \\
& \quad=\left(\Delta_{h}\left(\frac{\partial^{2} D}{\partial \varepsilon^{2}}(\varepsilon(v(t))) \varepsilon\left(v_{, k}(t)\right)\right), \varepsilon\left(\Delta_{h} v_{, k}(t)\right)\right) .
\end{aligned}
$$

Поскольку

$$
\begin{aligned}
\Delta_{h} & \left(\frac{\partial^{2} D}{\partial \varepsilon^{2}}(\varepsilon(v(t))) \varepsilon\left(v_{, k}(t)\right)\right) \\
& =\frac{\partial^{2} D}{\partial \varepsilon^{2}}(\varepsilon(v(t+h))) \varepsilon\left(\Delta_{h} v_{, k}(t)\right)+\Delta_{h}\left(\frac{\partial^{2} D}{\partial \varepsilon^{2}}(\varepsilon(v(t)))\right) \varepsilon\left(v_{, k}(t)\right),
\end{aligned}
$$


то в силу $\left(1.2_{3}\right)$ и (1.17) получим

$$
\begin{gathered}
-\left(\operatorname{div} \Delta_{h} \sigma(t), \widehat{w}(t)\right) \geqslant 2 \nu\left\|\nabla \varepsilon\left(\Delta_{h} v(t)\right)\right\|^{2}-C_{1}\left\|\nabla \varepsilon\left(\Delta_{h}(v(t))\right)\right\| \\
\quad \times\left\||\nabla \varepsilon(v(t))|\left|\varepsilon\left(\Delta_{h} v(t)\right)\right|\right\| \\
\stackrel{(2.1)}{\geqslant} \frac{\nu}{2}\left\|\nabla^{2} \Delta_{h} v(t)\right\|^{2}-C_{2}\left\|\nabla^{2} v(t)\right\|_{4}^{2}\left\|\nabla \Delta_{h} v(t)\right\|_{4}^{2} \\
\quad \stackrel{(2.6)}{\geqslant} \frac{\nu}{2}\left\|\nabla^{2} \Delta_{h} v(t)\right\|^{2}-C_{3} \Gamma^{\frac{1}{2}}(t)\left\|\nabla \Delta_{h} v(t)\right\|\left\|\nabla^{2} \Delta_{h} v(t)\right\| \\
\geqslant \frac{\nu}{4}\left\|\nabla^{2} \Delta_{h} v(t)\right\|^{2}-C_{4} \Gamma(t)\left\|\nabla \Delta_{h} v(t)\right\|^{2}
\end{gathered}
$$

где $\Gamma(t) \equiv\left\|\nabla^{2} v(t)\right\|_{4}^{4}$. Отметим, что в силу уже доказанной оценки (1.28) можно утверждать, что

$$
\begin{aligned}
\int_{\delta_{1}}^{T}\left\|\nabla^{2} v(t)\right\|_{4}^{4} d t & \leqslant C \underset{\delta_{1} \leqslant t \leqslant T}{\operatorname{ess} \sup _{T}}\left\|\nabla^{2} v(t)\right\|^{2}\left\|\nabla^{3} v\right\|_{Q_{\delta_{1}}, T}^{2} \\
& \leqslant \Phi_{8}^{2}\left(\ldots,\|\operatorname{curl} g\|_{Q_{\delta, T}}, \frac{1}{\delta_{1}-\delta}, \frac{1}{\delta}\right), \quad \delta_{1}>\delta>0 .
\end{aligned}
$$

Перейдем к оценке правой части в (11.15). Имеем

$$
\left(\Delta_{h}\left(v_{k}(t) v_{, k}(t)\right), \widehat{w}(t)\right)=\left(\Delta_{h}\left(v_{k}(t) \operatorname{curl} v_{, k}(t)\right), \operatorname{curl} \Delta_{h} v(t)\right)=B_{1}(t)+B_{2}(t)
$$

где

$$
\begin{aligned}
& B_{1}(t) \equiv\left(v_{k}(t+h) \operatorname{curl} \Delta_{h} v_{, k}(t), \operatorname{curl} \Delta_{h} v(t)\right)=0, \\
& B_{2}(t) \equiv\left(\operatorname{curl} v_{, k}(t) \Delta_{h} v_{k}(t), \operatorname{curl} \Delta_{h} v(t)\right), \\
&\left|B_{2}(t)\right| \leqslant C_{1}\left\|\nabla \Delta_{h} v(t)\right\|\left\|\nabla^{2} v(t)\right\|_{4}\left\|\Delta_{h} v(t)\right\|_{4} \stackrel{(2.6)}{\leqslant} C_{2}\left\|\nabla \Delta_{h} v(t)\right\|^{\frac{3}{2}} \Gamma^{\frac{1}{4}}(t)\left\|\Delta_{h} v(t)\right\|^{\frac{1}{2}} \\
& \stackrel{(2.5)}{\leqslant} C_{3}\left\|\nabla \Delta_{h} v(t)\right\|^{2} \Gamma^{\frac{1}{4}}(t) \leqslant C_{4}\left\|\nabla \Delta_{h} v(t)\right\|^{2}(\Gamma(t)+1) .
\end{aligned}
$$

И, наконец,

$$
\left(\Delta_{h} g(t), \widehat{w}(t)\right) \leqslant C\left\|\Delta_{h} g(t)\right\|\left\|\nabla^{2} \Delta_{h} v(t)\right\| .
$$

Принимая во внимание соотношения (11.17)-(11.23), выводим из (11.15), (11.16) неравенство

$$
\partial_{t}\left\|\nabla \Delta_{h} v(t)\right\|^{2}+\frac{\nu}{4}\left\|\nabla^{2} \Delta_{h} v(t)\right\|^{2} \leqslant C\left\{\left\|\Delta_{h} g(t)\right\|^{2}+\left\|\nabla \Delta_{h} v(t)\right\|^{2}(\Gamma(t)+1)\right\} .
$$

Неравенство (11.24) выполнено для п.в. $t \in[0, T]$ таких, что $t, t+h \in[0, T]$. 
Пусть теперь $\chi$ - срезающая функция, определяемая соотношениями (7.28). Тогда из (11.24) следует неравенство

$$
\begin{aligned}
& \partial_{t}\left(\chi(t)\left\|\nabla \Delta_{h} v(t)\right\|^{2}\right)+\frac{\nu}{4}\left(\chi(t)\left\|\nabla^{2} \Delta_{h} v(t)\right\|^{2}\right) \\
& \quad \leqslant C\left(\chi(t)\left\|\nabla \Delta_{h} v(t)\right\|^{2}\right)(\Gamma(t)+1)+\chi^{\prime}(t)\left\|\nabla \Delta_{h} v(t)\right\|^{2}+C \chi(t)\left\|\Delta_{h} g(t)\right\|^{2} .
\end{aligned}
$$

В свою очередь (11.25) влечет оценку

$$
\begin{aligned}
& \underset{0 \leqslant t \leqslant T-h}{\operatorname{ess} \sup _{0}}\left(\chi(t)\left\|\nabla \Delta_{h} v(t)\right\|^{2}\right) \\
& \leqslant C \int_{0}^{T-h}\left[\chi^{\prime}(t)\left\|\nabla \Delta_{h} v(t)\right\|^{2}+\chi(t)\left\|\partial_{t} g(t)\right\|^{2}\right] d t \\
& \quad \times \exp t\left\{C\left(\int_{\frac{\delta_{1}+\delta}{2}}^{T} \Gamma(t) d t+T\right)\right\} .
\end{aligned}
$$

С другой стороны, предполагая, что $h<\frac{\delta_{1}-\delta}{4}$, имеем

$$
\begin{aligned}
& \int_{0}^{T-h}\left(\chi^{\prime}(t)\left\|\nabla \Delta_{h} v(t)\right\|^{2}+\chi(t)\left\|\Delta_{h} g(t)\right\|^{2}\right) d x \\
& \quad \leqslant \frac{2}{\delta_{1}-\delta} \int_{\frac{\delta_{1}+\delta}{2}}^{T-h}\left\|\nabla \Delta_{h} v(t)\right\|^{2} d t+\int_{\frac{\delta_{1}+\delta}{2}}^{T-h}\left\|\Delta_{h} g(t)\right\|^{2} d x \\
& \quad \leqslant \frac{2}{\delta_{1}-\delta} \int_{\delta}^{T}\left\|\nabla \partial_{t} v(t)\right\|^{2} d t+\int_{\delta}^{T}\left\|\partial_{t} g(t)\right\|^{2} d x \\
& \quad \leqslant \frac{2}{\left(\delta_{1}-\delta\right) \delta^{2}} \int_{\delta}^{T}\left\|t \nabla \partial_{t} v(t)\right\|^{2} d x+\frac{1}{\delta^{5}} \int_{\delta}^{t} t^{5}\left\|\partial_{t} g(t)\right\|^{2} d t \\
& \quad \leqslant \frac{2}{\left(\delta_{1}-\delta\right) \delta^{2}} \Phi_{2}+\frac{1}{\delta^{5}}\left\|t^{\frac{5}{2}} \partial_{t} g\right\|_{Q_{T}}^{2} .
\end{aligned}
$$

Таким образом, правая часть в (11.26) мажорируется величиной, не зависящей от $h$. Используя (11.25)-(11.27), мы можем оценить $\int_{0}^{T-h} \chi(t)\left\|\nabla^{2} \Delta_{h} v(t)\right\|^{2} d t$ и получить

$$
\underset{\delta_{1} \leqslant t \leqslant T-h}{\operatorname{ess} \sup _{1}}\left\|\nabla \Delta_{h} v(t)\right\|^{2}+\int_{\delta_{1}}^{T-h}\left\|\nabla^{2} \Delta_{h} v(t)\right\|^{2} d x \leqslant \widetilde{\Phi}_{9}
$$

где $\widetilde{\Phi}_{9}$ зависят от тех же аргументов, что и $\Phi_{9}$, и не зависят от $h$. Устремляя $h \mathrm{k}$ нулю, получаем оценку (1.29). Теорема 1.8 доказана.

\section{Список литературы}

1. Ладыженская О.А. О нелинейных проблемах механики сплошных сред // Тр. Международного конгресса математиков (Москва, 1966). М.: Наука, 1968. С.560-573. 
2. Ладыженская О.А. Новые уравнения для описания движения вязких несжимаемых жидкостей и глобальная разрешимость краевых задач для них // Тр. Матем. ин-та им. В.А. Стеклова. 1967. Т. 102. С. 85-104.

3. Лады женская О. А. О некоторых модификациях уравнений Навье-Стокса для больших градиентов скоростей // Зап. научн. семинаров ЛОМИ. 1968. Т. 7. С. 126-154.

4. Ladyzhenskaya O.A., Seregin G.A. On global stability of the two-dimensional visco-plastic flows // Jyväskyllä - St.Petersbug Seminar on PDE's and Numerical Methods. Ber. Univ. Jyväskyllä Math. Inst. 1993. V. 56. P. 43-52.

5. Ladyzhenskaya O.A., Seregin G.A. On semigroups generated by initial boundary-value problems describing two-dimensional visco-plastic flows. Nonlinear evolution equations // Amer. Math. Soc. Transl., Amer. Math. Soc. Providence. Ser. 2. 1995. V. 164. P. 99-123.

6. Ladyzhenskaya O. A. Attractors for semi-groups and evolution equations. Lenzioni Lincee, Roma 1988. Cambridge: Cambridge Univ. Press, 1991.

7. Ладыженская О.А. О динамической системе, порожденной уравнениями Навье-Стокса // Зап. научн. семинаров ЛОМИ. 1972. Т. 27. С. 91-114.

8. Серегин Г. А. Течение двумерной обобщенной ньютоновской жидкости // Алгебра и анализ. 1997. Т. 9. №1. С. 163-196.

9. Лады женская $O . A$. Некоторые дополнения и уточнения к моим публикациям по теории аттракторов // Зап. научн. семинаров ЛОМИ. 1990. Т. 182. С. 102-112.

10. Douady A., Oesterlé J. Dimension de Hausdorf des attractors // C.R. Acad. Sc. 1980. V. 290. P. $1135-1138$.

11. Бабин А.В., Вишик М.И. Аттракторы эволюционных уравнений с частными производными и оценки их размерности // УМН. 1983. Т. 38. № 4. С. 134-187.

12. Лады женская О.А. Об оценках фрактальной размерности и числа определяющих мод для инвариантных множеств динамических систем // Зап. научн. семинаров ЛОМИ. 1987. T. 163 . C. $105-129$.

13. Ладыженская О.А. Решение "в целом" краевой задачи для уравнений Навье-Стокса в случае двух пространственных переменных // ДАН СССР. 1958. Т. 123. С. 427-429.

14. Лады женская О. А. Математические вопросы динамики вязкой несжимаемой жидкости. М.: Физматгиз, 1951; 2-е изд. М.: Наука, 1970.

15. Ладыженская О. А., Солонников В. А., Уральцева Н. Н. Линейные и квазилинейные уравнения параболического типа. М.: Наука, 1967.

16. Ладыженская О.А., Уральцева Н. Н. Линейные и квазилинейные уравнения эллиптического типа. М.: Наука, 1973.

17. Giaquinta $M$. Multiple integrals in the calculus of variations and nonlinear elliptic systems // Ann. Math. Studies. № 105. Princeton-N.Y.: Princeton University Press, 1983.

Санкт-Петербургское отделение

Поступило в редакцию

Математического института

22.IV.1997

им. В.А. Стеклова РАН,

Санкт-Петербург 